\title{
The Crank-Nicolson/Explicit Scheme for the Natural Convection Equations with Nonsmooth Initial Data
}

\author{
Jinting Yang, Hongxia Liang and Tong Zhang* \\ School of Mathematics E Information Science, Henan Polytechnic University, Jiaozuo, \\ Henan 454003, China
}

Received 10 July 2019; Accepted (in revised version) 10 June 2020

\begin{abstract}
In this article, a Crank-Nicolson/Explicit scheme is designed and analyzed for the time-dependent natural convection problem with nonsmooth initial data. The Galerkin finite element method (FEM) with stable MINI element is used for the velocity and pressure and linear polynomial for the temperature. The time discretization is based on the Crank-Nicolson scheme. In order to simplify the computations, the nonlinear terms are treated by the explicit scheme. The advantages of our numerical scheme can be list as follows: (1) The original problem is split into two linear subproblems, these subproblems can be solved in each time level in parallel and the computational sizes are smaller than the origin one. (2) A constant coefficient linear discrete algebraic system is obtained in each subproblem and the computation becomes easy. The main contributions of this work are the stability and convergence results of numerical solutions with nonsmooth initial data. Finally, some numerical results are presented to verify the established theoretical results and show the performances of the developed numerical scheme.
\end{abstract}

AMS subject classifications: 65M10, 65N30, 76Q10

Key words: Natural-Convection equations, Crank-Nicolson/Explicit scheme, nonsmooth initial data, error estimates.

\section{Introduction}

In this paper, we consider the following natural convection equations:

*Corresponding author.

Email: zhangtong0616@163.com (T. Zhang) 


$$
\begin{cases}\mathbf{u}_{t}-v \Delta \mathbf{u}+(\mathbf{u} \cdot \nabla) \mathbf{u}+\nabla p=-\kappa v^{2} j T+\mathbf{f} & \text { in } \Omega \times\left(0, T_{\text {time }}^{\text {final }}\right], \\ \operatorname{div} \mathbf{u}=0 & \text { in } \Omega \times\left(0, T_{\text {time }}^{\text {final }}\right], \\ T_{t}-\operatorname{Pr}^{-1} v \Delta T+\mathbf{u} \cdot \nabla T=g & \text { in } \Omega \times\left(0, T_{\text {time }}^{\text {final }}\right], \\ \mathbf{u}=0, \quad T=0 & \text { on } \partial \Omega \times\left(0, T_{\text {time }}^{f i n a l}\right], \\ \mathbf{u}(x, 0)=\mathbf{u}_{0}, \quad T(x, 0)=T_{0} & \text { on } \Omega \times\{0\},\end{cases}
$$

where $\mathbf{u}, T, p$ are the velocity, temperature and the pressure, $\mathbf{f}$ and $g$ are the body forces, $\Omega$ is a bounded convex polygonal domain, the parameters $v, \kappa$ and $\operatorname{Pr}$ are the viscosity, Groshoff and Prandtl numbers, $j=(0,1)^{T}$ is the vector of gravitational acceleration, $\mathrm{T}_{\text {time }}^{\text {final }}>0$ is the final time.

The natural convection problem is an important system with dissipative nonlinear terms in atmospheric dynamics (see [4,25]), it not only inherits all difficulties of the Navier-Stokes equations, but also contains strong coupling among variables and nonlinear terms. Hence, finding the numerical solutions becomes a difficult task, and several efficient numerical methods have been developed in recent years, for examples, $[5,8,27]$ for the discontinuous methods, [24,26] for the lattice Boltzmann method, [7] for the stabilized method, [21,23] for the iterative schemes.

As a classical second order scheme, the Crank-Nicolson scheme has been used to treat various problems. Here we just refer to [20,22] for the linear problems, [3] for the semilinear parabolic problem and the reference therein as the examples. Generally speaking, the implicit scheme for nonlinear term is unconditionally stable and has optimal error estimates, but we need to treat a nonlinear problem at each step and a lot of computing cost is required. In order to simplify the computations, some variants of the Crank-Nicolson scheme were developed, for examples, the Crank-Nicolson extrapolation scheme $[12,14,28]$, the Crank-Nicolson/Newton scheme [9]. The explicit scheme for nonlinear term is another way to treat the nonlinear term, one of the most important advantages is that the discrete algebraic system with a constant coefficient matrix is obtained at each time level. However, a restriction on the time-step was required. For examples we can refer to the Crank-Nicolson/Adams-Bashforth scheme [10, 14, 16, 31] and the references therein.

In this paper, we consider the Crank-Nicolson/Explicit scheme for the natural convection equations with nonsmooth initial data. In this way, the origin problem is split into two linear subproblems, and these subproblems with the constant coefficient matrix can be solved easily in each time level. Compared with [32,33], the main contributions can be list as follows:

(1) Under some restrictions on time step, almost unconditional stability results of numerical solutions in various norms are established with nonsmooth initial data.

(2) By introducing the weight function and using the negative norm technique, under the same time step conditions, we obtain that the Crank-Nicolson/Explicit 
scheme has the same convergence as the Crank-Nicolson extrapolation and CrankNicolson/Adams-Bashforth schemes, but the Crank-Nicolson/Explicit scheme implements easier.

(3) The Crank-Nicolson/Explicit scheme splits the origin problem into two linear subproblems and these subproblems can be solved in parallel. Furthermore, the computational size is reduced and the computational cost is saved.

The rest of this article is organized as follows. In Section 2, some basic assumptions and results of the natural convection problem are presented, the spatial semidiscrete numerical scheme and the corresponding stability and convergence results are also provided. Section 3 is devoted to develop the Crank-Niconlson/Explicit scheme for the natural convection equations, the stability results of numerical solutions with nonsmooth initial data are established. Convergence results of numerical solutions are provided in Section 4. Finally, Section 5 provides some numerical results to show the performances of the considered numerical scheme.

\section{Preliminaries}

\subsection{Weak form and some basic results}

In this paper, the standard Sobolev spaces and norms are used [1]. For example, the space $L^{2}(\Omega)^{d},(d=1,2)$ is associated with the usual $L^{2}$-scalar product $(\cdot)$ and $L^{2}$-norm $\|\cdot\|_{0}$. For convenience, we use the notations

$$
\begin{aligned}
& X=H_{0}^{1}(\Omega)^{2}, \quad W=H_{0}^{1}(\Omega), \quad M=L_{0}^{2}(\Omega)=\left\{\varphi \in L^{2}(\Omega): \int_{\Omega} \varphi d x=0\right\}, \\
& Z=L^{2}(\Omega), \quad V=\{\mathbf{v} \in X:(\nabla \cdot \mathbf{v}, q)=0, \forall q \in M\}, \\
& Y=L^{2}(\Omega)^{2}, \quad H=\left\{\mathbf{v} \in Y, \nabla \cdot \mathbf{v}=0,\left.\mathbf{v} \cdot n\right|_{\partial \Omega}=0\right\} .
\end{aligned}
$$

The spaces $W$ and $X$ are equipped with the usual product and norm $\|\nabla \mathbf{u}\|_{0}^{2}=(\nabla \mathbf{u}, \nabla \mathbf{u})$.

Set $A=-P \Delta$, where $P$ is the $L^{2}$-orthogonal projection of $Z$ onto $W$ or $Y$ onto $H$. Assume that $\Omega$ is such that of the domain of $A$ is given by (see $[12,17,30]$ )

$$
D(A)=H^{2}(\Omega)^{2} \cap V \text { and } Q(A)=H^{2}(\Omega) \cap W .
$$

The following assumption about the prescribed data for problem (1.1) is needed.

Assumption 2.1. (A1) The initial data $\mathbf{u}_{0}(x), T_{0}(x)$ and the force $\mathbf{f}, g$ satisfy

$$
\begin{aligned}
& \sup _{0 \leq t \leq T_{\text {time }}^{\text {final }}}\left\{\|\mathbf{f}(t)\|_{1}+\left\|\mathbf{f}_{t}(t)\right\|_{0}+\left\|\mathbf{f}_{t t}(t)\right\|_{0}+\|g(t)\|_{1}+\left\|g_{t}(t)\right\|_{0}+\left\|g_{t t}(t)\right\|_{0}\right\} \leq \widetilde{C}, \\
& \left\{\begin{array}{lll}
\left\|\nabla \mathbf{u}_{0}\right\|_{0}+\left\|\nabla T_{0}\right\|_{0}+\left\|\mathbf{u}_{0}\right\|_{L^{\infty}}+\left\|T_{0}\right\|_{L^{\infty}} \leq \widetilde{C}, & \mathbf{u}_{0} \in V \cap L^{\infty}(\Omega)^{2}, & T_{0} \in H^{1}(\Omega) \cap L^{\infty}(\Omega), \\
\left\|\nabla \mathbf{u}_{0}\right\|_{0}+\left\|\nabla T_{0}\right\|_{0} \leq \widetilde{C}, & \mathbf{u}_{0} \in V, \quad T_{0} \in H^{1},
\end{array}\right.
\end{aligned}
$$

where $\widetilde{C}$ is a general positive constant. 
Define the bilinear forms $a(\cdot, \cdot), \tilde{a}(\cdot, \cdot)$ and $d(\cdot, \cdot)$ on $X \times X, W \times W$ and $X \times M$ by

$$
a(\mathbf{u}, \mathbf{v})=v(\nabla \mathbf{u}, \nabla \mathbf{v}), \quad d(\mathbf{v}, q)=(q, \operatorname{div} \mathbf{v}), \quad \tilde{a}(T, \psi)=\operatorname{Pr}^{-1} v(\nabla T, \nabla \psi),
$$

the trilinear forms $b(\mathbf{u}, \mathbf{v}, \mathbf{w})$ and $\tilde{b}(\mathbf{u}, T, \psi)$ on $X \times X \times X$ and $X \times W \times W$ are

$$
\begin{aligned}
& b(\mathbf{u}, \mathbf{v}, \mathbf{w})=((\mathbf{u} \cdot \nabla) \mathbf{v}, \mathbf{w})+\frac{1}{2}((\operatorname{div} \mathbf{u}) \mathbf{v}, \mathbf{w})=\frac{1}{2}((\mathbf{u} \cdot \nabla) \mathbf{v}, \mathbf{w})-\frac{1}{2}((\mathbf{u} \cdot \nabla) \mathbf{w}, \mathbf{v}), \\
& \tilde{b}(\mathbf{u}, T, \psi)=((\mathbf{u} \cdot \nabla) T, \psi)+\frac{1}{2}((\operatorname{div} \mathbf{u}) T, \psi)=\frac{1}{2}((\mathbf{u} \cdot \nabla) T, \psi)-\frac{1}{2}((\mathbf{u} \cdot \nabla) \psi, T) .
\end{aligned}
$$

With above notations, for all $(\mathbf{v}, q, \psi) \in X \times M \times W, 0 \leq t \leq T_{\text {time }}^{\text {final }}$, the weak form of natural convection equations (1.1) aims to find $(\mathbf{u}, p, T) \in X \times M \times W$, such that

$$
\left\{\begin{array}{l}
\left(\mathbf{u}_{t}, \mathbf{v}\right)+a(\mathbf{u}, \mathbf{v})-d(\mathbf{v}, p)+d(\mathbf{u}, q)+b(\mathbf{u}, \mathbf{u}, \mathbf{v})=(\mathbf{f}, \mathbf{v})-\kappa v^{2}(j T, \mathbf{v}), \\
\left(T_{t}, \psi\right)+\tilde{a}(T, \psi)+\tilde{b}(\mathbf{u}, T, \psi)=(g, \psi), \\
\mathbf{u}(x, 0)=\mathbf{u}_{0}, \quad T(x, 0)=T_{0},\left.\quad \mathbf{u}_{t}\right|_{\partial \Omega}=0,\left.\quad T_{t}\right|_{\partial \Omega}=0 .
\end{array}\right.
$$

The following results can be found in Chapter 5 of Reference [25].

Theorem 2.1. Under the assumption of $\partial \Omega \in C^{2}$ or $\Omega \in \mathbb{R}^{2}$ is a convex polygon and $T_{0} \in$ $C^{1}\left(0, T_{\text {time }}^{\text {final }} ; \Omega\right)$ with $\boldsymbol{f}=0, g=0$, problem $(2.1)$ has at least a solution $(\boldsymbol{u}, p, T) \in L^{2}\left(0, T_{\text {time }}^{\text {final }} ; X\right) \cap$ $H^{1}\left(0, T_{\text {time }}^{\text {final }} ; V\right) \times L^{2}\left(0, T_{\text {time }}^{\text {final }} ; M\right) \times H^{1}\left(0, T_{\text {time }}^{\text {final }} ; W\right)$. In addition, the solution is unique provided that $v^{-1} \bar{N}^{2}\|\nabla T\|_{0}^{2}+2 v N\|\nabla \boldsymbol{u}\|_{0} \leq 2$, and the following prior estimate holds

$$
\|\boldsymbol{u}\|_{0}^{2}+\|T\|_{0}^{2}+\int_{0}^{T_{\text {time }}^{\text {final }}}\left(\|\nabla \boldsymbol{u}\|_{0}^{2}+\|\nabla T\|_{0}^{2}\right) d s \leq C \int_{0}^{T_{\text {time }}^{f i n a l}}\left(\left\|T_{0 t}\right\|_{0}^{2}+\left\|\nabla T_{0}\right\|_{0}^{2}\right) d s,
$$

where

$$
N=\sup _{\boldsymbol{u}, \boldsymbol{v}, \boldsymbol{w} \in X} \frac{b(\boldsymbol{u}, \boldsymbol{v}, \boldsymbol{w})}{\|\nabla \boldsymbol{u}\|_{0}\|\nabla \boldsymbol{v}\|_{0}\|\nabla \boldsymbol{w}\|_{0}}, \quad \bar{N}=\sup _{\boldsymbol{u} \in X, T, \psi \in W} \frac{\tilde{b}(\boldsymbol{u}, T, \psi)}{\|\nabla \boldsymbol{u}\|_{0}\|\nabla T\|_{0}\|\nabla \psi\|_{0}} .
$$

\subsection{Spatial discrete Galerkin finite element method}

Take a positive parameter $h \rightarrow 0$ and define a decomposition $J_{h}=J_{h}(\Omega)$ be a family of regular partitioning of triangles $K$ or quadrilaterals $K$ of the domain $\bar{\Omega}$. Based on $J_{h}$, we construct the conforming finite element spaces of $X_{h} \times M_{h} \times W_{h}$ of $X \times M \times W$. Furthermore, some assumptions are made about the spaces $X_{h}, M_{h}$ and $W_{h}$ (see $[2,6,11,29]$ ).

Assumption 2.2. (A2) For each $\mathbf{v} \in D(A)$ and $\psi \in Q(A)$ and $q \in H^{1}(\Omega) \cap M$, there are the approximations $\pi_{h} \mathbf{v} \in V_{h}, \mu_{h} \psi \in W_{h}$ and $\rho_{h} q \in M_{h}$ such that

$$
\left\|\nabla\left(\mathbf{v}-\pi_{h} \mathbf{v}\right)\right\|_{0} \leq c h\|A \mathbf{v}\|_{0}, \quad\left\|\nabla\left(\psi-\mu_{h} \psi\right)\right\|_{0} \leq c h\|A \psi\|_{0}, \quad\left\|q-\rho_{h} q\right\|_{0} \leq c h\|\nabla q\|_{0} .
$$


For each $\phi_{h} \in X_{h}$ or $W_{h}$, the following inverse inequalities hold

$$
\left\|\nabla \phi_{h}\right\|_{0} \leq c h^{-1}\left\|\phi_{h}\right\|_{0}, \quad\left\|\phi_{h}\right\|_{L^{\infty}} \leq c h^{-1}\left\|\phi_{h}\right\|_{0} .
$$

Define the discrete analogue of the space $V_{h}$ as

$$
V_{h}=\left\{\mathbf{v}_{h} \in X_{h} ; d\left(\mathbf{v}_{h}, q_{h}\right)=0, \forall q_{h} \in M_{h}\right\},
$$

and set $P_{h}^{1}: Y \rightarrow V_{h}$ and $P_{h}^{2}: Z \rightarrow W_{h}$ be the $L^{2}$-orthogonal projections defined by

$$
\begin{array}{ll}
\left(P_{h}^{1} \mathbf{v}, \mathbf{v}_{h}\right)=\left(\mathbf{v}, \mathbf{v}_{h}\right), & \mathbf{v} \in Y, \quad \mathbf{v}_{h} \in V_{h} \\
\left(P_{h}^{2} \psi, \psi_{h}\right)=\left(\psi, \psi_{h}\right), & \psi \in Z, \quad \psi_{h} \in W_{h} .
\end{array}
$$

Assumption 2.3. (A3) There exists a constant $\beta>0$ such that

$$
\beta\left\|q_{h}\right\|_{0} \leq \sup _{0 \neq \mathbf{v}_{h} \in X_{h}} \frac{\left|d\left(\mathbf{v}_{h}, q_{h}\right)\right|}{\left\|\mathbf{v}_{h}\right\|_{1}}, \quad \forall q_{h} \in M_{h}
$$

The following properties are classical for $i=1,2$ (see $[11,19])$

$$
\begin{aligned}
& \left\|\nabla P_{h}^{i} \varphi\right\|_{0} \leq \gamma\|\nabla \varphi\|_{0}, \quad\left\|\varphi-P_{h}^{i} \varphi\right\|_{0} \leq \gamma h\left\|\nabla\left(\varphi-P_{h}^{i} \varphi\right)\right\|_{0}, \quad \varphi \in X \text { or } W, \\
& \left\|\varphi-P_{h}^{i} \varphi\right\|_{0}+h\left\|\nabla\left(\varphi-P_{h}^{i} \varphi\right)\right\|_{0} \leq \gamma h^{2}\|A \varphi\|_{0}, \quad \varphi \in D(A) \text { or } Q(A),
\end{aligned}
$$

for some positive constants $\gamma$.

With above notations, for all $\left(\mathbf{v}_{h}, q_{h}, \psi_{h}\right) \in X_{h} \times M_{h} \times W_{h}$ and $0 \leq t \leq T_{\text {time }}^{\text {final }}$, the Galerkin finite element method for problem (2.1) is to seek $\left(\mathbf{u}_{h}, p_{h}, T_{h}\right) \in X_{h} \times M_{h} \times W_{h}$ such that

$$
\left\{\begin{array}{l}
\left(\mathbf{u}_{h t}, \mathbf{v}_{h}\right)+a\left(\mathbf{u}_{h}, \mathbf{v}_{h}\right)-d\left(\mathbf{v}_{h}, p_{h}\right)+d\left(\mathbf{u}_{h}, q_{h}\right)+b\left(\mathbf{u}_{h}, \mathbf{u}_{h}, \mathbf{v}_{h}\right)=\left(\mathbf{f}, \mathbf{v}_{h}\right)-\kappa v^{2}\left(j T_{h}, \mathbf{v}_{h}\right), \\
\left(T_{h t}, \psi_{h}\right)+\tilde{a}\left(T_{h}, \psi_{h}\right)+\tilde{b}\left(\mathbf{u}_{h}, T_{h}, \psi_{h}\right)=\left(g, \psi_{h}\right), \\
\mathbf{u}_{h}(x, 0)=\mathbf{u}_{0 h}=P_{h} \mathbf{u}_{0}, \quad T_{h}(x, 0)=T_{0 h}=P_{h} T_{0}, \\
\left.\mathbf{u}_{h t}\right|_{\partial \Omega}=0,\left.\quad T_{h t}\right|_{\partial \Omega}=0 .
\end{array}\right.
$$

With the help of $P_{h}^{i}$, we define the discrete analogue $A_{i h}=-P_{h}^{i} \Delta_{h}(i=1,2)$ and $\Delta_{h}$ by $\left(-\Delta_{h} \phi_{h}, \varphi_{h}\right)=\left(\nabla \phi_{h}, \nabla \varphi_{h}\right), \forall \phi_{h}, \varphi_{h} \in X_{h}$ or $W_{h}$ with the "discrete" Sobolev norms $\left\|\phi_{h}\right\|_{r}=$ $\left\|A_{i h}^{r / 2} \phi_{h}\right\|_{0}$ for $r=-1,0,1,2$ and $\phi_{h} \in X_{h}$ or $W_{h}$. Furthermore, it holds

$$
\left\|\phi_{h}\right\|_{0} \leq \gamma_{0}\left\|\phi_{h}\right\|_{1}, \quad\left\|\phi_{h}\right\|_{1} \leq \gamma_{0}\left\|A_{i h} \phi_{h}\right\|_{0} .
$$

The following properties of trilinear terms can be found in $[12,13,15,18]$. 
Lemma 2.1. The trilinear forms $b(\cdot, \cdot, \cdot)$ and $\tilde{b}(\cdot, \cdot, \cdot)$ satisfy

$$
\begin{aligned}
& b\left(\boldsymbol{u}_{h}, \boldsymbol{v}_{h}, \mathbf{w}_{h}\right)=-b\left(\boldsymbol{u}_{h}, \mathbf{w}_{h}, \boldsymbol{v}_{h}\right), \quad\left|b\left(\boldsymbol{u}_{h}, \boldsymbol{v}_{h}, \mathbf{w}_{h}\right)\right| \leq \widehat{C}|\log h|^{1 / 2}\left\|\nabla \boldsymbol{u}_{h}\right\|_{0}\left\|\nabla \boldsymbol{v}_{h}\right\|_{0}\left\|_{\mathbf{w}_{h} \|_{0},}, \quad \tilde{b}\left(\boldsymbol{u}_{h}, T_{h}, \psi_{h}\right)=-\tilde{b}\left(\boldsymbol{u}_{h}, \psi_{h}, T_{h}\right), \quad\left|\tilde{b}\left(\boldsymbol{u}_{h}, T_{h}, \psi_{h}\right)\right| \leq \widetilde{C}|\log h|^{1 / 2}\right\| \nabla \boldsymbol{u}_{h}\left\|_{0}\right\| \nabla T_{h}\left\|_{0}\right\| \psi_{h} \|_{0}, \\
& \left|b\left(\boldsymbol{u}_{h}, \boldsymbol{v}_{h}, \mathbf{w}_{h}\right)\right|+\left|b\left(\boldsymbol{v}_{h}, \boldsymbol{u}_{h}, \mathbf{w}_{h}\right)\right|+\left|b\left(\mathbf{w}_{h}, \boldsymbol{u}_{h}, \boldsymbol{v}_{h}\right)\right| \\
& \leq \frac{\widehat{C}}{2}\left\|\boldsymbol{u}_{h}\right\|_{0}^{1 / 2}\left\|\boldsymbol{u}_{h}\right\|_{1}^{1 / 2}\left\|\boldsymbol{v}_{h}\right\|_{1}\left\|\mathbf{w}_{h}\right\|_{0}^{1 / 2}\left\|\mathbf{w}_{h}\right\|_{1}^{1 / 2}+\frac{\widehat{C}}{2}\left\|\boldsymbol{u}_{h}\right\|_{1}\left\|\boldsymbol{v}_{h}\right\|_{0}^{1 / 2}\left\|\boldsymbol{v}_{h}\right\|_{1}^{1 / 2}\left\|\mathbf{w}_{h}\right\|_{0}^{1 / 2}\left\|\mathbf{w}_{h}\right\|_{1}^{1 / 2}, \\
& \left|\tilde{b}\left(\boldsymbol{u}_{h}, T_{h}, \psi_{h}\right)\right|+\left|\tilde{b}\left(T_{h}, \boldsymbol{u}_{h}, \psi_{h}\right)\right|+\left|\tilde{b}\left(\psi_{h}, \boldsymbol{u}_{h}, T_{h}\right)\right| \leq \widetilde{C}\left\|\boldsymbol{u}_{h}\right\|_{0}^{1 / 2}\left\|\boldsymbol{u}_{h}\right\|_{1}^{1 / 2}\left\|T_{h}\right\|_{1}\left\|\psi_{h}\right\|_{0}^{1 / 2}\left\|\psi_{h}\right\|_{1}^{1 / 2}, \\
& \left|b\left(\boldsymbol{u}_{h}, \boldsymbol{v}_{h}, \mathbf{w}_{h}\right)\right|+\left|b\left(\boldsymbol{v}_{h}, \boldsymbol{u}_{h}, \mathbf{w}_{h}\right)\right|+\left|b\left(\mathbf{w}_{h}, \boldsymbol{u}_{h}, \boldsymbol{v}_{h}\right)\right| \\
& \leq \frac{\widehat{C}}{2}\left\|A_{1 h} \boldsymbol{v}_{h}\right\|_{0}^{1 / 2}\left\|\boldsymbol{v}_{h}\right\|_{1}^{1 / 2}\left\|\boldsymbol{u}_{h}\right\|_{0}^{1 / 2}\left\|\boldsymbol{u}_{h}\right\|_{1}^{1 / 2}\left\|\mathbf{w}_{h}\right\|_{0}+\frac{\widehat{C}}{2}\left\|A_{1 h} \boldsymbol{v}_{h}\right\|_{0}^{1 / 2}\left\|\boldsymbol{v}_{h}\right\|_{0}^{1 / 2}\left\|\boldsymbol{u}_{h}\right\|_{1}\left\|\mathbf{w}_{h}\right\|_{0}, \\
& \left|\tilde{b}\left(\boldsymbol{u}_{h}, T_{h}, \psi_{h}\right)\right|+\left|\tilde{b}\left(T_{h}, \boldsymbol{u}_{h}, \psi_{h}\right)\right|+\left|\tilde{b}\left(\psi_{h}, \boldsymbol{u}_{h}, T_{h}\right)\right| \\
& \leq \frac{\widetilde{C}}{2}\left\|A_{2 h} T_{h}\right\|_{0}^{1 / 2}\left\|T_{h}\right\|_{1}^{1 / 2}\left\|\boldsymbol{u}_{h}\right\|_{0}^{1 / 2}\left\|\boldsymbol{u}_{h}\right\|_{1}^{1 / 2}\left\|\psi_{h}\right\|_{0}+\frac{\widetilde{C}}{2}\left\|A_{2 h} T_{h}\right\|_{0}^{1 / 2}\left\|T_{h}\right\|_{0}^{1 / 2}\left\|\boldsymbol{u}_{h}\right\|_{1}\left\|\psi_{h}\right\|_{0},
\end{aligned}
$$

for all $\boldsymbol{u}_{h}, \boldsymbol{v}_{h}, \in V_{h}, \mathbf{w}_{h} \in X_{h}$ and $T_{h}, \psi_{h} \in W_{h}$, where $\widehat{C}>0$ and $\widetilde{C}>0$ are constants.

In order to present the error analysis for time discretization, we recall the following smooth properties of $\mathbf{u}_{h}$ and $T_{h}$, and some errors of $\mathbf{u}-\mathbf{u}_{h}, T-T_{h}$ and $p-p_{h}$. Following the techniques used in [13-16], we can obtain the following properties.

Theorem 2.2. Under the assumptions (A1)-(A3), for all $t \in\left[0, T_{\text {time }}^{\text {final }}\right]$, then the numerical solution $\left(\boldsymbol{u}_{h}, T_{h}\right)$ of problem (2.3) satisfies

$$
\begin{aligned}
& \left\|\boldsymbol{u}_{h}(t), T_{h}(t)\right\|_{0}^{2}+\left\|\boldsymbol{u}_{h}(t), T_{h}(t)\right\|_{1}^{2}+\sigma(t)\left\|A_{h} \boldsymbol{u}_{h}(t), A_{h} T_{h}(t)\right\|_{0}^{2} \\
& \quad+\int_{0}^{t}\left\{\left\|\nabla \boldsymbol{u}_{h}, \nabla T_{h}\right\|_{0}^{2}+\left\|A_{h} \boldsymbol{u}_{h}, A_{h} T_{h}\right\|_{0}^{2}\right\} d s \leq C, \quad \sigma^{1+r}(t)\left\|\boldsymbol{u}_{h t}, T_{h t}\right\|_{r}^{2} \leq C, \quad r=-1,0,1,2, \\
& \int_{0}^{t}\left\{\left\|\boldsymbol{u}_{h s}, T_{h s}\right\|_{0}^{2}+\sigma^{r}(s)\left\|\boldsymbol{u}_{h s}, T_{h s}\right\|_{r}^{2}\right\} d s \leq C, \quad r=1,2, \\
& \left.\int_{0}^{t}\left\{\sigma^{2-r}(s)\left\|A_{h}^{-r / 2} \boldsymbol{u}_{h s s}, A_{h}^{-r / 2} T_{h s s}\right\|_{0}^{2}\right)\right\} d s \leq C, \quad r=0,1,2, \\
& \sigma^{3}(t)\left\|\boldsymbol{u}_{h t t}(t), T_{h t t}(t)\right\|_{0}^{2}+\int_{0}^{t}\left\{\sigma^{3}(s)\left(\left\|\boldsymbol{u}_{h s s}, T_{h s s}\right\|_{1}^{2}+\left\|\boldsymbol{u}_{h s s s}, T_{h s s s}\right\|_{-1}^{2}\right)\right\} d s \leq C,
\end{aligned}
$$

where

$$
\begin{aligned}
& \sigma(t)=\min \{1, t\}, \quad\left\|\boldsymbol{u}_{h}(t), T_{h}(t)\right\|_{r}=\left\|\boldsymbol{u}_{h}(t)\right\|_{r}^{2}+\left\|T_{h}(t)\right\|_{r}^{2} \quad \text { with } r=-1,0,1, \\
& \left\|A_{h} \boldsymbol{u}_{h}(t), A_{h} T_{h}(t)\right\|_{0}=\left\|A_{h} \boldsymbol{u}_{h}(t)\right\|_{0}^{2}+\left\|A_{h} T_{h}(t)\right\|_{0}^{2} .
\end{aligned}
$$

Theorem 2.3. Under the assumptions (A1)-(A3), for all $0<t \leq T_{\text {time }}^{\text {final }}$, it holds

$$
\sigma^{1 / 2}(t)\left\|\boldsymbol{u}-\boldsymbol{u}_{h}, T-T_{h}\right\|_{0} \leq C h^{2}, \quad \sigma^{1 / 2}(t)\left\|\boldsymbol{u}-\boldsymbol{u}_{h}, T-T_{h}\right\|_{1}+\sigma(t)\left\|p-p_{h}\right\|_{0} \leq C h .
$$


Lemma 2.2 ([12]). Let $C^{\prime}$ and $\Delta t, a_{k}, b_{k}, d_{k}$ be non-negative numbers for integers $k \geq 0$. If

$$
a_{n}+\Delta t \sum_{k=r}^{n} b_{k} \leq \Delta t \sum_{k=r-1}^{n-1} d_{k} a_{k}+C^{\prime}, \quad \forall n \geq r-1
$$

Then

$$
a_{n}+\Delta t \sum_{k=r}^{n} b_{k} \leq C^{\prime} \exp \left(\Delta t \sum_{k=r-1}^{n-1} d_{k}\right), \quad \forall n \geq r-1
$$

\section{The Crank-Nicolson/Explicit scheme for the natural convection equations}

In this section, we denote the time step $\Delta t=\frac{T_{\text {time }}^{\text {final }}}{N}$ with $N$ is an integer and set $t_{n}=n \Delta t$. Let

$$
\mathbf{u}_{h}^{0}=\mathbf{u}_{0 h}=P_{h} \mathbf{u}_{0}, \quad T_{h}^{0}=T_{0 h}=P_{h} T_{0} .
$$

Firstly, we find $\left(\mathbf{u}_{h}^{1}, p_{h}^{1}, T_{h}^{1}\right) \in X_{h} \times M_{h} \times W_{h}$ by the Euler-backward scheme with the explicit scheme to treat the nonlinear terms

$$
\left\{\begin{array}{l}
\left(d_{t} \mathbf{u}_{h}^{1}, \mathbf{v}_{h}\right)+a\left(\mathbf{u}_{h}^{1}, \mathbf{v}_{h}\right)-d\left(\mathbf{v}_{h}, p_{h}^{1}\right)+d\left(\mathbf{u}_{h}^{1}, q_{h}\right)+b\left(\mathbf{u}_{h}^{0}, \mathbf{u}_{h}^{0}, \mathbf{v}_{h}\right) \\
\quad=\left(\mathbf{f}\left(t_{1}\right), \mathbf{v}_{h}\right)-\kappa v^{2}\left(j T_{h}^{0}, \mathbf{v}_{h}\right), \\
\left(d_{t} T_{h}^{1}, \psi_{h}\right)+\tilde{a}\left(T_{h}^{1}, \psi_{h}\right)+\tilde{b}\left(\mathbf{u}_{h}^{0}, T_{h}^{0}, \psi_{h}\right)=\left(g\left(t_{1}\right), \psi_{h}\right)
\end{array}\right.
$$

for all $\left(\mathbf{v}_{h}, q_{h}, \psi_{h}\right) \in X_{h} \times M_{h} \times W_{h}$, where $d_{t} \phi_{h}^{n}=\frac{\phi_{h}^{n}-\phi_{h}^{n-1}}{\Delta t}, \phi$ can take $\mathbf{u}$ and $T$.

Based on the numerical solution $\left(\mathbf{u}_{h}^{1}, p_{h}^{1}, T_{h}^{1}\right)$, for all $\left(\mathbf{v}_{h}, q_{h}, \psi_{h}\right) \in X_{h} \times M_{h} \times W_{h}$, find $\left(\mathbf{u}_{h}^{n}, p_{h}^{n}, T_{h}^{n}\right) \in X_{h} \times M_{h} \times W_{h}$ with $n=2, \cdots, N$ by

$$
\left\{\begin{array}{c}
\left(d_{t} \mathbf{u}_{h}^{n}, \mathbf{v}_{h}\right)+a\left(\overline{\mathbf{u}}_{h}^{n}, \mathbf{v}_{h}\right)-d\left(\mathbf{v}_{h}, p_{h}^{n}\right)+d\left(\overline{\mathbf{u}}_{h}^{n}, q_{h}\right)+b\left(\mathbf{u}_{h}^{n-1}, \mathbf{u}_{h}^{n-1}, \mathbf{v}_{h}\right) \\
=\left(\overline{\mathbf{f}}\left(t_{h}\right), \mathbf{v}_{h}\right)-\kappa v^{2}\left(j \bar{T}_{h}^{n}, \mathbf{v}_{h}\right), \\
\left(d_{t} T_{h}^{n}, \psi_{h}\right)+\tilde{a}\left(\bar{T}_{h}^{n}, \psi_{h}\right)+\tilde{b}\left(\mathbf{u}_{h}^{n-1}, T_{h}^{n-1}, \psi_{h}\right)=\left(\bar{g}\left(t_{n}\right), \psi_{h}\right) .
\end{array}\right.
$$

Here and below, we use the following notations frequently

$$
\bar{\phi}_{h}^{n}=\frac{\phi_{h}^{n}+\phi_{h}^{n-1}}{2}, \quad \bar{\phi}_{h}\left(t_{n}\right)=\frac{\phi_{h}\left(t_{n}\right)+\phi_{h}\left(t_{n-1}\right)}{2}, \text { where } \phi \text { can take } \mathbf{u} \text { or } T \text {. }
$$


From Eqs. (3.1) and (3.2), we obtain

$$
\begin{aligned}
& \left\{\begin{array}{l}
\left(\mathbf{u}_{h}^{1}, \mathbf{v}_{h}\right)+\Delta t\left[a\left(\mathbf{u}_{h}^{1}, \mathbf{v}_{h}\right)-d\left(\mathbf{v}_{h}, p_{h}^{1}\right)\right] \\
=\left(\mathbf{u}_{h}^{0}, \mathbf{v}_{h}\right)+\Delta t\left[\left(\mathbf{f}\left(t_{1}\right), \mathbf{v}_{h}\right)-\kappa v^{2}\left(j T_{h}^{0}, \mathbf{v}_{h}\right)-b\left(\mathbf{u}_{h}^{0}, \mathbf{u}_{h}^{0}, \mathbf{v}_{h}\right)\right], \\
d\left(\mathbf{u}_{h}^{1}, q_{h}\right)=0, \\
\left(T_{h}^{1}, \psi_{h}\right)+\Delta t \tilde{a}\left(T_{h}^{1}, \psi_{h}\right)=\left(T_{h}^{0}, \psi_{h}\right)+\Delta t\left[\left(g\left(t_{1}\right), \psi_{h}\right)-\tilde{b}\left(\mathbf{u}_{h}^{0}, T_{h}^{0}, \psi_{h}\right)\right],
\end{array}\right. \\
& \left\{\begin{array}{l}
\left(\mathbf{u}_{h}^{n}, \mathbf{v}_{h}\right)+\Delta t\left[\frac{1}{2} a\left(\mathbf{u}_{h}^{n}, \mathbf{v}_{h}\right)-d\left(\mathbf{v}_{h}, p_{h}^{n}\right)\right]=\left(\mathbf{u}_{h}^{n-1}, \mathbf{v}_{h}\right) \\
\quad+\Delta t\left[\left(\overline{\mathbf{f}}\left(t_{h}\right), \mathbf{v}_{h}\right)-\frac{1}{2} a\left(\mathbf{u}_{h}^{n-1}, \mathbf{v}_{h}\right)-\kappa v^{2}\left(j \bar{T}_{h}^{n}, \mathbf{v}_{h}\right)-b\left(\mathbf{u}_{h}^{n-1}, \mathbf{u}_{h}^{n-1}, \mathbf{v}_{h}\right)\right], \\
d\left(\overline{\mathbf{u}}_{h}^{n}, q_{h}\right)=0, \\
\left(T_{h}^{n}, \psi_{h}\right)+\Delta t \frac{1}{2} \tilde{a}\left(T_{h}^{n}, \psi_{h}\right) \\
=\left(T_{h}^{n-1}, \psi_{h}\right)+\Delta t\left[\left(\bar{g}\left(t_{h}\right), \psi_{h}\right)-\frac{1}{2} \tilde{a}\left(T_{h}^{n-1}, \psi_{h}\right)-\tilde{b}\left(\mathbf{u}_{h}^{n-1}, T_{h}^{n-1}, \psi_{h}\right)\right] .
\end{array}\right.
\end{aligned}
$$

For the given $\left(u_{h}^{0}, T_{h}^{0}\right) \in X_{h} \times W_{h}$, set

$$
\bar{A}\left(T_{h}^{1}, \psi_{h}\right)=\left(T_{h}^{1}, \psi_{h}\right)+\Delta t \tilde{a}\left(T_{h}^{1}, \psi_{h}\right) .
$$

Taking $\psi_{h}=T_{h}^{1}$ in $\bar{A}(\cdot, \cdot)$, one finds that

$$
\bar{A}\left(T_{h}^{1}, T_{h}^{1}\right)=\left\|T_{h}^{1}\right\|_{0}^{2}+\Delta t \operatorname{Pr}^{-1} v\left\|\nabla T_{h}^{1}\right\|_{0}^{2} \geq \alpha\left\|T_{h}^{1}\right\|_{1}^{2},
$$

where $\alpha=\min \left\{1, \Delta t \operatorname{Pr}^{-1} v\right\}$ and $\bar{A}(\cdot, \cdot)$ is coercive. Hence, the third equation of problem (3.3a) has a unique solution $T_{h}^{1} \in W_{h}$.

Furthermore, for the given $\left(\mathbf{u}_{h}^{0}, T_{h}^{0}\right) \in X_{h} \times W_{h}$, denote

$$
\bar{B}\left(\mathbf{u}_{h}^{1}, \mathbf{v}_{h}\right)=\left(\mathbf{u}_{h}^{1}, \mathbf{v}_{h}\right)+\Delta t a\left(\mathbf{u}_{h}^{1}, \mathbf{v}_{h}\right) .
$$

Then the first equation of problem (3.3a) can be rewritten as

$$
\bar{B}\left(\mathbf{u}_{h}^{1}, \mathbf{v}_{h}\right)-\Delta t d\left(\mathbf{v}_{h}, p_{h}^{1}\right)=\left(\mathbf{u}_{h}^{0}, \mathbf{v}_{h}\right)+\Delta t\left[\left(\mathbf{f}\left(t_{1}\right), \mathbf{v}_{h}\right)-\kappa v^{2}\left(j T_{h}^{0}, \mathbf{v}_{h}\right)-b\left(\mathbf{u}_{h}^{0}, \mathbf{u}_{h}^{0}, \mathbf{v}_{h}\right)\right] .
$$

Choosing $\mathbf{v}_{h}=\mathbf{u}_{h}^{1}$ in $\bar{B}(\cdot, \cdot)$, we obtain

$$
\bar{B}\left(\mathbf{u}_{h}^{1}, \mathbf{u}_{h}^{1}\right)=\left\|\mathbf{u}_{h}^{1}\right\|_{0}^{2}+\Delta t v\left\|\nabla \mathbf{u}_{h}^{1}\right\|_{0}^{2} \geq \bar{\alpha}\left\|\mathbf{u}_{h}\right\|_{1}^{2}
$$

where $\bar{\alpha}=\min \{1, v \Delta t\}$ and $\bar{B}(\cdot, \cdot)$ is also coercive. While $d(\cdot, \cdot)$ satisfies the discrete inf-sup condition (A3), then the first and second equations in problem (3.3a) admit a unique solution $\left(\mathbf{u}_{h}^{1}, p_{h}^{1}\right) \in X_{h} \times M_{h}$. As a consequence, problem (3.1) has a unique solution $\left(\mathbf{u}_{h}^{1}, p_{h}^{1}, T_{h}^{1}\right) \in X_{h} \times M_{h} \times W_{h}$.

By the same analysis, we can establish the existence and uniqueness of numerical solutions in scheme (3.3b). It means that problem (3.2) admits a unique solution $\left(\mathbf{u}_{h}^{n}, p_{h}^{n}, T_{h}^{n}\right) \in X_{h} \times M_{h} \times W_{h}$. 
Theorem 3.1. Suppose that the assumptions (A1)-(A3) hold and the time step $\Delta t$ satisfies

$$
\begin{cases}c^{\prime} 2 \Delta t \leq 1, & \boldsymbol{u}_{0} \in V \cap L^{\infty}(\Omega)^{2}, \quad T_{0} \in W \cap L^{\infty}(\Omega), \\ c^{\prime}|\log h| \Delta t \leq 1, & \boldsymbol{u}_{0} \in V, \quad T_{0} \in W\end{cases}
$$

where $c^{\prime}>0$ is a constant, the numerical solution $\left(\boldsymbol{u}_{h}^{m}, T_{h}^{m}\right)(m=1,2)$ of schemes (3.1)-(3.2) satisfies

$$
\begin{aligned}
& \left\|\boldsymbol{u}_{h}^{m}, T_{h}^{m}\right\|_{0}^{2}+\min \left\{v, \operatorname{Pr}^{-1} v\right\} \sum_{n=1}^{m}\left\|\boldsymbol{u}_{h}^{n}, T_{h}^{n}\right\|_{1}^{2} \Delta t \leq C_{0}^{\prime}, \\
& \min \left\{v, \operatorname{Pr}^{-1} v\right\}\left\|\boldsymbol{u}_{h}^{m}, T_{h}^{m}\right\|_{1}^{2}+\min \left\{v^{2}, \operatorname{Pr}^{-2} v^{2}\right\}\left\|A_{h} \boldsymbol{u}_{h}^{n}, A_{h} T_{h}^{n}\right\|_{0}^{2}+\left\|p_{h}^{n}\right\|_{0}^{2} \leq C_{1}^{\prime}, \\
& \min \left\{v^{2}, \operatorname{Pr}^{-2} v^{2}\right\}\left\|A_{h} \boldsymbol{u}_{h}^{m}, A_{h} T_{h}^{m}\right\|_{0}^{2} \Delta t+\min \left\{v, \operatorname{Pr}^{-1} v\right\} \Delta t^{2} \sum_{n=1}^{m}\left\|d_{t} u_{h}^{n}, d_{t} T_{h}^{n}\right\|_{1}^{2} \leq C_{2}^{\prime},
\end{aligned}
$$

with some positive constants

$$
C_{0}^{\prime} \geq\left\|\boldsymbol{u}_{h}^{0}, T_{h}^{0}\right\|_{0}^{2}, \quad C_{1}^{\prime} \geq \min \left\{v, \operatorname{Pr}^{-1} v\right\}\left\|\boldsymbol{u}_{h}^{0}, T_{h}^{0}\right\|_{1}^{2} \quad \text { and } \quad C_{2}^{\prime} \geq C_{1}^{\prime},
$$

all these constants depend on the data $\mathrm{Pr}^{-1}, v, \kappa, \Omega, T_{\text {time }}^{\text {final }}, \boldsymbol{u}_{0}, T_{0}, f$ and $g$.

Proof. For $r=0,1$, taking $\mathbf{v}_{h}=2 A_{h}^{r} \mathbf{u}_{h}^{1} \Delta t \in V_{h}, \psi_{h}=2 A_{h}^{r} T_{h}^{1} \Delta t \in W_{h}, q_{h}=0$ in (3.1) and $\mathbf{v}_{h}=$ $2 A_{h}^{r} \mathbf{u}_{h}^{2} \Delta t \in V_{h}, \psi_{h}=2 A_{h}^{r} T_{h}^{2} \Delta t \in W_{h}, q_{h}=0$ in (3.2) with $n=2$, respectively, we get

$$
\begin{aligned}
& \left\{\begin{array}{c}
\left(d_{t} \mathbf{u}_{h}^{1}, 2 A_{h}^{r} \mathbf{u}_{h}^{1} \Delta t\right)+a\left(\mathbf{u}_{h}^{1}, 2 A_{h}^{r} \mathbf{u}_{h}^{1} \Delta t\right)+b\left(\mathbf{u}_{h}^{0}, \mathbf{u}_{h}^{0}, 2 A_{h}^{r} \mathbf{u}_{h}^{1} \Delta t\right) \\
\quad=\left(\mathbf{f}\left(t_{1}\right), 2 A_{h}^{r} \mathbf{u}_{h}^{1} \Delta t\right)-\kappa v^{2}\left(j T_{h}^{0}, 2 A_{h}^{r} \mathbf{u}_{h}^{1} \Delta t\right) \\
\left(d_{t} T_{h}^{1}, 2 A_{h}^{r} T_{h}^{1} \Delta t\right)+\tilde{a}\left(T_{h}^{1}, 2 A_{h}^{r} T_{h}^{1} \Delta t\right)+\tilde{b}\left(\mathbf{u}_{h}^{0}, T_{h}^{0}, 2 A_{h}^{r} T_{h}^{1} \Delta t\right)=\left(g\left(t_{1}\right), 2 A_{h}^{r} T_{h}^{1} \Delta t\right)
\end{array}\right. \\
& \left\{\begin{array}{c}
\left(d_{t} \mathbf{u}_{h}^{2}, 2 A_{h}^{r} \mathbf{u}_{h}^{2} \Delta t\right)+a\left(\overline{\mathbf{u}}_{h}^{2}, 2 A_{h}^{r} \mathbf{u}_{h}^{2} \Delta t\right)+b\left(\mathbf{u}_{h}^{1}, \mathbf{u}_{h}^{1}, 2 A_{h}^{r} \mathbf{u}_{h}^{2} \Delta t\right) \\
=\left(\overline{\mathbf{f}}\left(t_{2}\right), 2 A_{h}^{r} \mathbf{u}_{h}^{2} \Delta t\right)-\kappa v^{2}\left(j \bar{T}_{h}^{2}, 2 A_{h}^{r} \mathbf{u}_{h}^{2} \Delta t\right) \\
\left(d_{t} T_{h}^{2}, 2 A_{h}^{r} T_{h}^{2} \Delta t\right)+\tilde{a}\left(\bar{T}_{h}^{2}, 2 A_{h}^{r} T_{h}^{2} \Delta t\right)+\tilde{b}\left(\mathbf{u}_{h}^{1}, T_{h}^{1}, 2 A_{h}^{r} T_{h}^{2} \Delta t\right)=\left(\bar{g}\left(t_{2}\right), 2 A_{h}^{r} T_{h}^{2} \Delta t\right)
\end{array}\right.
\end{aligned}
$$

Thanks to Lemma 2.2 and the Cauchy inequality, we have

$$
\left\{\begin{array}{l}
\left\|\mathbf{u}_{h}^{1}\right\|_{r}^{2}-\left\|\mathbf{u}_{h}^{0}\right\|_{r}^{2}+\left\|d_{t} \mathbf{u}_{h}^{1}\right\|_{r}^{2} \Delta t^{2}+2 v\left\|\mathbf{u}_{h}^{1}\right\|_{r+1}^{2} \Delta t \\
\leq v\left\|\mathbf{u}_{h}^{1}\right\|_{r+1}^{2} \Delta t+2 v^{-1} \gamma_{0}^{2(1-r)}\left\|\mathbf{f}\left(t_{1}\right)\right\|_{0}^{2} \Delta t+2 k^{2} v^{3} \gamma_{0}^{2(1-r)}\left\|T_{h}^{0}\right\|_{0}^{2} \Delta t \\
\quad-2 b\left(\mathbf{u}_{h}^{0}, \mathbf{u}_{h}^{0}, A_{h}^{r} \mathbf{u}_{h}^{1}\right) \Delta t \\
\left\|T_{h}^{1}\right\|_{r}^{2}-\left\|T_{h}^{0}\right\|_{r}^{2}+\left\|d_{t} T_{h}^{1}\right\|_{r}^{2} \Delta t^{2}+2 \operatorname{Pr}^{-1} v\left\|T_{h}^{1}\right\|_{r+1}^{2} \Delta t \\
\leq \frac{1}{2} \operatorname{Pr}^{-1} v\left\|T_{h}^{1}\right\|_{r+1}^{2} \Delta t+2 \operatorname{Prv}^{-1} \gamma_{0}^{2(1-r)}\left\|g\left(t_{1}\right)\right\|_{0}^{2} \Delta t-2 \tilde{b}\left(\mathbf{u}_{h}^{0}, T_{h}^{0}, A_{h}^{r} T_{h}^{1}\right) \Delta t
\end{array}\right.
$$




$$
\left\{\begin{array}{l}
\left\|\mathbf{u}_{h}^{2}\right\|_{r}^{2}-\left\|\mathbf{u}_{h}^{1}\right\|_{r}^{2}+\left\|d_{t} \mathbf{u}_{h}^{2}\right\|_{r}^{2} \Delta t^{2}+\frac{v}{2}\left(\left\|\mathbf{u}_{h}^{2}\right\|_{r+1}^{2}-\left\|\mathbf{u}_{h}^{1}\right\|_{r+1}^{2}\right) \Delta t \\
\leq \frac{v}{8}\left\|\mathbf{u}_{h}^{2}\right\|_{r+1}^{2} \Delta t+16 v^{-1} \gamma_{0}^{2(1-r)}\left\|\overline{\mathbf{f}}\left(t_{2}\right)\right\|_{0}^{2} \Delta t+16 k^{2} v^{3} \gamma_{0}^{2(1-r)}\left\|\bar{T}_{h}^{2}\right\|_{0}^{2} \Delta t \\
\quad-2 b\left(\mathbf{u}_{h}^{1}, \mathbf{u}_{h}^{1}, A_{h}^{r} \mathbf{u}_{h}^{2}\right) \Delta t \\
\leq \frac{v}{8}\left\|\mathbf{u}_{h}^{2}\right\|_{r+1}^{2} \Delta t+16 v^{-1} \gamma_{0}^{2(1-r)}\left\|\overline{\mathbf{f}}\left(t_{2}\right)\right\|_{0}^{2} \Delta t+16 k^{2} v^{3} \gamma_{0}^{2(1-r)}\left\|\bar{T}_{h}^{2}\right\|_{0}^{2} \Delta t \\
\quad-2 b\left(\mathbf{u}_{h}^{1}, \mathbf{u}_{h}^{1}, A_{h}^{r} \mathbf{u}_{h}^{2}\right) \Delta t+2\left|b\left(\mathbf{u}_{h}^{0}, \mathbf{u}_{h}^{0}, A_{h}^{r} \mathbf{u}_{h}^{2}\right)\right| \Delta t, \\
\left\|T_{h}^{2}\right\|_{r}^{2}-\left\|T_{h}^{1}\right\|_{r}^{2}+\left\|d_{t} T_{h}^{2}\right\|_{r}^{2} \Delta t^{2}+\frac{P r^{-1} v}{2}\left(\left\|T_{h}^{2}\right\|_{r+1}^{2}-\left\|T_{h}^{1}\right\|_{r+1}^{2}\right) \Delta t \\
\leq \frac{P r^{-1} v}{16}\left\|T_{h}^{2}\right\|_{r+1}^{2} \Delta t+\frac{16 \gamma_{0}^{2(1-r)}}{\operatorname{Pr}^{-1} v}\left\|\bar{g}\left(t_{2}\right)\right\|_{0}^{2} \Delta t-2 \tilde{b}\left(\mathbf{u}_{h}^{1}, T_{h}^{1}, A_{h}^{r} T_{h}^{2}\right) \Delta t \\
\leq \frac{P r^{-1} v}{16}\left\|T_{h}^{2}\right\|_{r+1}^{2} \Delta t+\frac{16 \gamma_{0}^{2(1-r)}}{P r^{-1} v}\left\|\bar{g}\left(t_{2}\right)\right\|_{0}^{2} \Delta t \\
\quad-2 \tilde{b}\left(\mathbf{u}_{h}^{1}, T_{h}^{1}, A_{h}^{r} T_{h}^{2}\right) \Delta t+2\left|\tilde{b}\left(\mathbf{u}_{h}^{0}, T_{h}^{0}, A_{h}^{r} T_{h}^{2}\right)\right| \Delta t .
\end{array}\right.
$$

For $r=0,1$ and $i=1,2$, using Lemma 2.2, (2.2a) and (2.4), we obtain

$$
\begin{aligned}
& 2\left|b\left(\mathbf{u}_{h}^{0}, \mathbf{u}_{h}^{0}, A_{h}^{r} \mathbf{u}_{h}^{i}\right)\right| \Delta t \leq \frac{v}{8}\left\|\mathbf{u}_{h}^{i}\right\|_{r+1}^{2} \Delta t+\frac{2 \widehat{C} \gamma_{0}^{2(1-r)}}{v}|\log h|^{r}\left\|\mathbf{u}_{h}^{0}\right\|_{1}^{4} \Delta t, \quad \mathbf{u}_{0} \in V, \\
& 2\left|b\left(\mathbf{u}_{h}^{0}, \mathbf{u}_{h}^{0}, A_{h}^{r} \mathbf{u}_{h}^{i}\right)\right| \Delta t \\
& \leq 2\left|\left(\left(\left(\mathbf{u}_{h}^{0}-\mathbf{u}_{0}\right) \cdot \nabla\right) \mathbf{u}_{h}^{0}, A_{h}^{r} \mathbf{u}_{h}^{i}\right)\right| \Delta t+\left|\left(\operatorname{div} \mathbf{u}_{h}^{0}\left(\mathbf{u}_{h}^{0}-\mathbf{u}_{0}\right), A_{h}^{r} \mathbf{u}_{h}^{i}\right)\right| \Delta t \\
& \quad+2\left|\left(\left(\mathbf{u}_{0} \cdot \nabla\right) \mathbf{u}_{h}^{0}, A_{h}^{r} \mathbf{u}_{h}^{i}\right)\right| \Delta t+\left|\left(\operatorname{div} \mathbf{u}_{h}^{0} \cdot \mathbf{u}_{0}, A_{h}^{r} \mathbf{u}_{h}^{i}\right)\right| \Delta t \\
& \leq c\left\|\mathbf{u}_{h}^{0}-\mathbf{u}_{0}\right\|_{0}\left\|\mathbf{u}_{h}^{0}\right\|_{1}\left\|A_{h}^{r} \mathbf{u}_{h}^{i}\right\|_{L^{\infty}} \Delta t+c\left\|\mathbf{u}_{0}\right\|_{L^{\infty}}\left\|\mathbf{u}_{h}^{0}\right\|_{1}\left\|A_{h}^{r} \mathbf{u}_{h}^{i}\right\|_{0} \Delta t \\
& \leq \frac{v}{8}\left\|\mathbf{u}_{h}^{i}\right\|_{r+1}^{2} \Delta t+c\left(\left\|\nabla \mathbf{u}_{0}\right\|_{0}^{2}+\left\|\mathbf{u}_{0}\right\|_{L^{\infty}}^{2}\right)\left\|\mathbf{u}_{h}^{0}\right\|_{1}^{2} \Delta t, \quad \mathbf{u}_{0} \in V \cap L^{\infty}(\Omega)^{2}, \\
& 2\left|b\left(\mathbf{u}_{h}^{1}, \mathbf{u}_{h}^{1}, A_{h}^{r} \mathbf{u}_{h}^{2}\right)\right| \Delta t \leq \frac{v}{8}\left\|\mathbf{u}_{h}^{2}\right\|_{r+1}^{2} \Delta t+\frac{v}{8}\left\|A_{h} \mathbf{u}_{h}^{1}\right\|_{0}^{2} \Delta t+c\left\|\mathbf{u}_{h}^{1}\right\|_{0}^{2}\left\|\mathbf{u}_{h}^{1}\right\|_{1}^{4} \Delta t, \\
& 2\left|\tilde{b}\left(\mathbf{u}_{h}^{0}, T_{h}^{0}, A_{h}^{r} T_{h}^{i}\right)\right| \Delta t \\
& \leq \quad \widetilde{C} \gamma_{0}^{1-r}|\log h|^{r / 2}\left\|\mathbf{u}_{h}^{0}\right\|_{1}\left\|T_{h}^{0}\right\|_{1}\left\|T_{h}^{i}\right\|_{r+1} \Delta t \\
& \leq \frac{\operatorname{Pr}^{-1} v}{8}\left\|T_{h}^{i}\right\|_{r+1}^{2} \Delta t+\frac{2 \widetilde{C}_{0}^{2(1-r)}}{P r^{-1} v}|\log h|^{r}\left\|\mathbf{u}_{h}^{0}\right\|_{1}^{2}\left\|T_{h}^{0}\right\|_{1}^{2} \Delta t, \quad\left(\mathbf{u}_{0}, T_{0}\right) \in V \times W, \\
& 2\left|\tilde{b}\left(\mathbf{u}_{h}^{0}, T_{h}^{0}, A_{h}^{r} T_{h}^{i}\right)\right| \Delta t \\
& \leq 2\left|\left(\left(\left(\mathbf{u}_{h}^{0}-\mathbf{u}_{0}\right) \cdot \nabla\right) T_{h}^{0}, A_{h}^{r} T_{h}^{i}\right)\right| \Delta t+\left|\left(\operatorname{div} \mathbf{u}_{h}^{0}\left(T_{h}^{0}-T_{0}\right), A_{h}^{r} T_{h}^{i}\right)\right| \Delta t \\
& \quad+2\left|\left(\left(\mathbf{u}_{0} \cdot \nabla\right) T_{h}^{0}, A_{h}^{r} T_{h}^{i}\right)\right| \Delta t+\left|\left(\operatorname{div} \mathbf{u}_{h}^{0} \cdot T_{0}, A_{h}^{r} T_{h}^{i}\right)\right| \Delta t \\
& \leq c\left\|\mathbf{u}_{h}^{0}-\mathbf{u}_{0}\right\|_{0}\left\|T_{h}^{0}\right\|_{1}\left\|A_{h}^{r} T_{h}^{i}\right\|_{L^{\infty}} \Delta t+c\left\|\mathbf{u}_{0}\right\|_{L^{\infty}}\left\|T_{h}^{0}\right\|_{1}\left\|A_{h}^{r} T_{h}^{i}\right\|_{0} \Delta t \\
& \leq \frac{P r^{-1} v}{8}\left\|T_{h}^{i}\right\|_{r+1}^{2} \Delta t+c\left(\left\|\nabla \mathbf{u}_{0}\right\|_{0}^{2}+\left\|\mathbf{u}_{0}\right\|_{L^{\infty}}^{2}\right)\left\|T_{h}^{0}\right\|_{1}^{2} \Delta t, \quad \mathbf{u}_{0} \in V \cap L^{\infty}(\Omega)^{2}, \quad T_{0} \in W \cap L^{\infty}(\Omega)
\end{aligned}
$$




$$
\begin{aligned}
& 2\left|\tilde{b}\left(\mathbf{u}_{h}^{1}, T_{h}^{1}, A_{h}^{r} T_{h}^{2}\right)\right| \Delta t \\
& \leq \frac{3}{2} \widetilde{C} \gamma_{0}^{1-r}\left\|\mathbf{u}_{h}^{1}\right\|_{0}^{\frac{1}{2}}\left\|T_{h}^{1}\right\|_{1}\left\|A_{h} \mathbf{u}_{h}^{1}\right\|_{0}^{\frac{1}{2}}\left\|T_{h}^{2}\right\|_{r+1} \Delta t \\
& \leq \frac{P^{-1} v}{8}\left\|T_{h}^{2}\right\|_{r+1}^{2} \Delta t+\frac{P^{-1} v}{8}\left\|A_{h} \mathbf{u}_{h}^{1}\right\|_{0}^{2} \Delta t+c\left\|\mathbf{u}_{h}^{1}\right\|_{0}^{2}\left\|T_{h}^{1}\right\|_{1}^{4} \Delta t
\end{aligned}
$$

Combining above inequalities with (3.6a) and (3.6b), one finds

$$
\begin{aligned}
& \left\{\begin{array}{l}
\left\|\mathbf{u}_{h}^{1}\right\|_{r}^{2}+\left\|d_{t} \mathbf{u}_{h}^{1}\right\|_{r}^{2} \Delta t^{2}+v\left\|\mathbf{u}_{h}^{1}\right\|_{r+1}^{2} \Delta t \\
\leq\left\|\mathbf{u}_{h}^{0}\right\|_{r}^{2}+c\left\|\mathbf{f}\left(t_{1}\right)\right\|_{0}^{2} \Delta t+c\left\|T_{h}^{0}\right\|_{0}^{2} \Delta t+c M_{1} \Delta t, \\
\left\|T_{h}^{1}\right\|_{r}^{2}+\left\|d_{t} T_{h}^{1}\right\|_{r}^{2} \Delta t^{2}+P r^{-1} v\left\|T_{h}^{1}\right\|_{r+1}^{2} \Delta t \\
\leq\left\|T_{h}^{0}\right\|_{r}^{2}+c\left\|g\left(t_{1}\right)\right\|_{0}^{2} \Delta t+c M_{1}^{\prime} \Delta t,
\end{array}\right. \\
& \left\{\begin{array}{l}
\left\|\mathbf{u}_{h}^{2}\right\|_{r}^{2}+\left\|d_{t} \mathbf{u}_{h}^{2}\right\|_{r}^{2} \tau^{2}+\frac{v}{4}\left\|\mathbf{u}_{h}^{2}\right\|_{r+1}^{2} \tau \\
\leq\left\|\mathbf{u}_{h}^{1}\right\|_{r}^{2}+\frac{v}{2}\left\|\mathbf{u}_{h}^{1}\right\|_{r+1}^{2}+v\left\|A_{h} \mathbf{u}_{h}^{1}\right\|_{0}^{2} \tau+c\left\|\overline{\mathbf{f}}\left(t_{2}\right)\right\|_{0}^{2} \tau \\
+c\left\|\bar{T}_{h}^{2}\right\|_{0}^{2} \tau+c\left\|\mathbf{u}_{h}^{1}\right\|_{0}^{2}\left\|\mathbf{u}_{h}^{1}\right\|_{1}^{4} \tau+c M_{1} \tau, \\
\left\|T_{h}^{2}\right\|_{r}^{2}+\left\|d_{t} T_{h}^{2}\right\|_{r}^{2} \tau^{2}+\frac{P r^{-1} v}{4}\left\|T_{h}^{2}\right\|_{r+1}^{2} \tau \\
\leq\left\|T_{h}^{1}\right\|_{r}^{2}+\frac{P r^{-1} v}{2}\left\|T_{h}^{1}\right\|_{r+1}^{2} \tau+P r^{-1} v\left\|A_{h} \mathbf{u}_{h}^{1}\right\|_{0}^{2} \tau \\
+c\left\|\bar{g}\left(t_{2}\right)\right\|_{0}^{2} \tau+c\left\|\mathbf{u}_{h}^{1}\right\|_{0}^{2}\left\|T_{h}^{1}\right\|_{1}^{4} \tau+c M_{1}^{\prime} \tau,
\end{array}\right.
\end{aligned}
$$

where

$$
\begin{aligned}
& M_{1}= \begin{cases}\left(\left\|\nabla \mathbf{u}_{0}\right\|_{0}^{2}+\left\|\mathbf{u}_{0}\right\|_{L^{\infty}}^{2}\right)\left\|\mathbf{u}_{h}^{0}\right\|_{1}^{2}, & \mathbf{u}_{0} \in V \cap L^{\infty}(\Omega)^{2}, \\
|\log h|^{r}\left\|\mathbf{u}_{h}^{0}\right\|_{1}^{4}, & \mathbf{u}_{0} \in V,\end{cases} \\
& M_{1}^{\prime}=\left\{\begin{array}{ll}
|\log h|^{r}\left\|\mathbf{u}_{h}^{0}\right\|_{1}^{2}\left\|T_{h}^{0}\right\|_{1}^{2}, & \left(\mathbf{u}_{0}, T_{0}\right) \in V \times W, \\
\left(\left\|\nabla \mathbf{u}_{0}\right\|_{0}^{2}+\left\|\mathbf{u}_{0}\right\|_{L^{\infty}}^{2}\right)\left\|T_{h}^{0}\right\|_{1}^{2}, & \mathbf{u}_{0} \in V \cap L^{\infty}(\Omega)^{2},
\end{array} \quad T_{0} \in W \cap L^{\infty}(\Omega),\right.
\end{aligned}
$$

for $r=0,1$.

By the assumption (A3), applying Lemma 2.2, (2.4), (3.1) and (3.2), we obtain

$$
\begin{aligned}
\left\|p_{h}^{1}\right\|_{0}^{2} \Delta t \leq c & \left(\left\|d_{t} \mathbf{u}_{h}^{1}\right\|_{0}^{2}+v^{2}\left\|A_{h} \mathbf{u}_{h}^{1}\right\|_{0}^{2}+\left\|\mathbf{f}\left(t_{1}\right)\right\|_{0}^{2}+\left\|T_{h}^{0}\right\|_{0}^{2}+M_{1}\right) \Delta t, \\
\left\|p_{h}^{2}\right\|_{0}^{2} \Delta t \leq c\left(\left\|d_{t} \mathbf{u}_{h}^{2}\right\|_{0}^{2}+v^{2}\left\|A_{h} \mathbf{u}_{h}^{2}\right\|_{0}^{2}+v^{2}\left\|A_{h} \mathbf{u}_{h}^{1}\right\|_{0}^{2}\right) \Delta t & +c\left(\left\|\overline{\mathbf{f}}\left(t_{2}\right)\right\|_{0}^{2}+\left\|\bar{T}_{h}^{2}\right\|_{0}^{2}+\left\|\mathbf{u}_{h}^{1}\right\|_{0}^{2}\left\|\mathbf{u}_{h}^{1}\right\|_{1}^{4}+M_{1}\right) \Delta t .
\end{aligned}
$$

Furthermore, by Lemma 2.2, (2.4), (3.1) and (3.2), we deduce that

$$
\begin{aligned}
& \left\|d_{t} T_{h}^{1}\right\|_{0}^{2} \Delta t \leq c\left(\operatorname{Pr}^{-2} v^{2}\left\|A_{h} T_{h}^{1}\right\|_{0}^{2}+\left\|g\left(t_{1}\right)\right\|_{0}^{2}+M_{1}^{\prime}\right) \Delta t, \\
& \left\|d_{t} T_{h}^{2}\right\|_{0}^{2} \Delta t \leq c\left(\operatorname{Pr}^{-2} v^{2}\left\|A_{h} T_{h}^{2}\right\|_{0}^{2}+\left\|A_{h} T_{h}^{1}\right\|_{0}^{2}+\left\|\bar{g}\left(t_{2}\right)\right\|_{0}^{2}+\left\|\mathbf{u}_{h}^{1}\right\|_{0}^{2}\left\|T_{h}^{1}\right\|_{1}^{4}+M_{1}^{\prime}\right) \Delta t .
\end{aligned}
$$

As a consequence, under the condition (3.4), we complete these proof. 
Theorem 3.2. Suppose that the assumptions (A1)-(A3) hold and the time step $\Delta t$ satisfies

$$
\begin{cases}\max \left\{\widetilde{C}_{3}, \widehat{C}_{3}\right\} \Delta t \leq 1, & \boldsymbol{u}_{0} \in V \cap L^{\infty}, \quad T_{0} \in W \cap L^{\infty}, \\ \max \left\{\widetilde{C}_{3}, \widehat{C}_{3}\right\}|\log h| \Delta t \leq \min \left\{1, C_{2}^{-2}\right\}, & \boldsymbol{u}_{0} \in V, \quad T_{0} \in W,\end{cases}
$$

where $\widehat{C}_{3}=128^{2} \gamma_{0}^{2} \widehat{C} v^{-5} C_{1} C_{2}\left(1+C_{2}^{2}\right), \widetilde{C}_{3}=\operatorname{Pr}^{5} \widehat{C}_{3}$. For all $1 \leq m \leq N$, we have

$$
\begin{aligned}
& \left\|\boldsymbol{u}_{h}^{m}, T_{h}^{m}\right\|_{0}^{2}+\min \left\{v, \operatorname{Pr}^{-1} v\right\} \Delta t \sum_{n=1}^{m}\left\|\overline{\boldsymbol{u}}_{h}^{n}, \bar{T}_{h}^{n}\right\|_{1}^{2} \leq C_{0}, \\
& \min \left\{v, \operatorname{Pr}^{-1} v\right\}\left\|\boldsymbol{u}_{h}^{m}, T_{h}^{m}\right\|_{1}^{2}+\Delta t \sum_{n=1}^{m}\left(\left\|d_{t} \boldsymbol{u}_{h}^{1}, d_{t} T_{h}^{1}\right\|_{0}^{2}+\left\|p_{h}^{n}\right\|_{0}^{2}\right. \\
& \left.\quad+\min \left\{v^{2}, \operatorname{Pr}^{-2} v^{2}\right\}\left\|A_{h} \overline{\boldsymbol{u}}_{h}^{n}, A_{h} \bar{T}_{h}^{n}\right\|_{0}^{2}\right) \leq C_{1}, \\
& \min \left\{v^{2}, \operatorname{Pr}^{-2} v^{2}\right\}\left\|A_{h} \boldsymbol{u}_{h}^{m}, A_{h} T_{h}^{m}\right\|_{0}^{2} \Delta t+\min \left\{v, \operatorname{Pr}^{-1} v\right\} \Delta t^{2} \sum_{n=1}^{m}\left\|d_{t} \boldsymbol{u}_{h}^{n}, d_{t} T_{h}^{n}\right\|_{1}^{2} \leq C_{2},
\end{aligned}
$$

where $C_{i} \geq C_{i}^{\prime},(i=0,1,2)$ are some constants depending on the data $\mathrm{Pr}^{-1}, v, \kappa, \Omega, T_{\text {time }}^{\text {final }}, \boldsymbol{u}_{0}, T_{0}$, $f, g$.

Proof. We inductive method to prove (3.10a)-(3.10c). From Theorem 3.1, we know that (3.10a)-(3.10c) hold with $m=1,2$. Then we assume that (3.10a)-(3.10c) hold for $m=3, \cdots, J-$ 1. We need to prove that (3.10a)-(3.10c) hold for $m=J$.

Taking $\psi_{h}=2 T_{h}^{n} \Delta t \in W_{h}, \mathbf{v}_{h}=0, q_{h}=0$ in (3.2), using Lemma 2.2 and the following facts

$$
\begin{aligned}
& \phi_{h}^{n}=\bar{\phi}_{h}^{n}+\frac{1}{2} d_{t} \phi_{h}^{n} \Delta t, \quad \phi_{h}^{n}=2 \bar{\phi}_{h}^{n}-\phi_{h}^{n-1}, \quad \phi_{h}^{n}=\bar{\phi}_{h}^{n-1}+d_{t} \phi_{h}^{n} \Delta t+\frac{1}{2} d_{t} \phi_{h}^{n-1} \Delta t, \\
& 2\left(d_{t} \phi_{h}^{n}, \phi_{h}^{n}\right) \Delta t=\left\|\phi_{h}^{n}\right\|_{0}^{2}-\left\|\phi_{h}^{n-1}\right\|_{0}^{2}+\left\|d_{t} \phi_{h}^{n}\right\|_{0}^{2} \Delta t^{2}, \\
& 2 a\left(\bar{\phi}_{h}^{n}, \phi_{h}^{n}\right) \Delta t=\frac{v}{2}\left(\left\|\phi_{h}^{n}\right\|_{1}^{2}-\left\|\phi_{h}^{n-1}\right\|_{1}^{2}+4\left\|\bar{\phi}_{h}^{n}\right\|_{1}^{2}\right) \Delta t, \phi \text { can take u or } T,
\end{aligned}
$$

we have

$$
\begin{aligned}
& \left(\left\|T_{h}^{n}\right\|_{0}^{2}+\frac{\operatorname{Pr}^{-1} v}{2}\left\|T_{h}^{n}\right\|_{1}^{2} \Delta t\right)-\left(\left\|T_{h}^{n-1}\right\|_{0}^{2}+\frac{\operatorname{Pr}^{-1} v}{2}\left\|T_{h}^{n-1}\right\|_{1}^{2} \Delta t\right) \\
& +\left\|d_{t} T_{h}^{n}\right\|_{0}^{2} \Delta t^{2}+2 \operatorname{Pr}^{-1} v\left\|\bar{T}_{h}^{n}\right\|_{1}^{2} \Delta t \\
= & \left(\bar{g}\left(t_{n}\right), 2 \bar{T}_{h}^{n}+d_{t} T_{h}^{n} \Delta t\right) \Delta t+\tilde{b}\left(\mathbf{u}_{h}^{n-1}, T_{h}^{n-1}, 2 d_{t} T_{h}^{n}\right) \Delta t^{2} .
\end{aligned}
$$

Thanks to the Cauchy inequality and Lemma 2.2, one finds

$$
\begin{aligned}
& \left|\left(\bar{g}\left(t_{n}\right), 2 \bar{T}_{h}^{n}+d_{t} T_{h}^{n} \Delta t\right)\right| \Delta t \\
& \leq \operatorname{Pr}^{-1} v\left\|\bar{T}_{h}^{n}\right\|_{1}^{2} \Delta t+\frac{1}{2}\left\|d_{t} T_{h}^{n}\right\|_{0}^{2} \Delta t^{2}+\left(\frac{\gamma_{0}^{2}}{\operatorname{Pr}^{-1} v}+\frac{1}{2} \Delta t\right)\left\|\bar{g}\left(t_{n}\right)\right\|_{0}^{2} \Delta t, \\
& 2\left|\tilde{b}\left(\mathbf{u}_{h}^{n-1}, T_{h}^{n-1}, d_{t} T_{h}^{n}\right)\right| \Delta t^{2} \leq \frac{1}{4} G^{\frac{1}{2}}\left(T_{h}^{n-1}\right)\left\|\mathbf{u}_{h}^{n-1}\right\|_{1}\left\|d_{t} T_{h}^{n}\right\|_{0} \Delta t^{2} \\
& \leq C G\left(T_{h}^{n-1}\right)\left\|\mathbf{u}_{h}^{n-1}\right\|_{1}^{2} \Delta t^{2}+\frac{1}{2}\left\|d_{t} T_{h}^{n}\right\|_{0}^{2} \Delta t^{2},
\end{aligned}
$$


where $G\left(\phi_{h}^{n}\right)=4^{2} \gamma_{0} \widetilde{C}\left\|\phi_{h}^{n}\right\|_{1}\left\|A_{h} \phi_{h}^{n}\right\|_{0}, \phi$ can take $\mathbf{u}$ and $T$.

Under the inductive assumption with $m=1, \cdots, J-1$, we get

$$
\begin{aligned}
& G\left(T_{h}^{n-1}\right) \Delta t \leq 4^{2} \gamma_{0} \widetilde{C}\left\|T_{h}^{n-1}\right\|_{1}\left\|A_{h} T_{h}^{n-1}\right\|_{0} \Delta t \\
\leq & 4^{2} \operatorname{Pr}^{3 / 2} v^{-3 / 2} \gamma_{0} \widetilde{C} C_{1}^{1 / 2} C_{2}^{1 / 2} \Delta t^{1 / 2} \leq \operatorname{Pr}^{-1} v .
\end{aligned}
$$

Combining above inequalities with (3.11), using the inductive assumption with $m=$ $1, \cdots, J-1$ and summing the inequality from $n=3$ to $n=J$, we have

$$
\begin{aligned}
& \left\|T_{h}^{J}\right\|_{0}^{2}+\operatorname{Pr}^{-1} v \Delta t\left\|T_{h}^{J}\right\|_{1}^{2}+\operatorname{Pr}^{-1} v \Delta t \sum_{n=3}^{J}\left\|\bar{T}_{h}^{n}\right\|_{1}^{2} \\
\leq & \left\|T_{h}^{2}\right\|_{0}^{2}+\frac{\operatorname{Pr}^{-1} v}{2}\left\|T_{h}^{2}\right\|_{1}^{2} \Delta t+\left(\frac{\gamma_{0}^{2}}{\operatorname{Pr}^{-1} v}+\frac{1}{2} \Delta t\right) T_{\text {time }}^{\text {final }} \sup _{0 \leq t \leq T_{\text {time }}^{\text {final }}}\|g(t)\|_{0}^{2}+C_{1} \Delta t .
\end{aligned}
$$

Choosing $\mathbf{v}_{h}=2 \mathbf{u}_{h}^{n} \Delta t \in V_{h}, \psi_{h}=0, q_{h}=0$ in (3.2), we get that

$$
\left(d_{t} \mathbf{u}_{h}^{n}, 2 \mathbf{u}_{h}^{n} \Delta t\right)+a\left(\overline{\mathbf{u}}_{h}^{n}, 2 \mathbf{u}_{h}^{n} \Delta t\right)+b\left(\mathbf{u}_{h}^{n-1}, \mathbf{u}_{h}^{n-1}, 2 \mathbf{u}_{h}^{n} \Delta t\right)=\left(\overline{\mathbf{f}}\left(t_{n}\right), 2 \mathbf{u}_{h}^{n} \Delta t\right)-\kappa v^{2}\left(j \bar{T}_{h}^{n}, 2 \mathbf{u}_{h}^{n} \Delta t\right) .
$$

Thanks to the Cauchy inequality and Lemma 2.2, we arrive at

$$
\begin{aligned}
& 2\left|b\left(\mathbf{u}_{h}^{n-1}, \mathbf{u}_{h}^{n-1}, \mathbf{u}_{h}^{n}\right)\right| \Delta t=2\left|b\left(\mathbf{u}_{h}^{n-1}, \mathbf{u}_{h}^{n-1}, d_{t} \mathbf{u}_{h}^{n}\right)\right| \Delta t^{2} \\
& \leq \frac{1}{4} G^{\frac{1}{2}}\left(\mathbf{u}_{h}^{n-1}\right)\left\|\mathbf{u}_{h}^{n-1}\right\|_{1}\left\|d_{t} \mathbf{u}_{h}^{n}\right\|_{0} \Delta t^{2} \leq C G\left(\mathbf{u}_{h}^{n-1}\right)\left\|\mathbf{u}_{h}^{n-1}\right\|_{1}^{2} \Delta t^{2}+\frac{1}{4}\left\|d_{t} \mathbf{u}_{h}^{n}\right\|_{0}^{2} \Delta t^{2}, \\
& \left|\left(\overline{\mathbf{f}}\left(t_{n}\right), 2 \overline{\mathbf{u}}_{h}^{n}+d_{t} \mathbf{u}_{h}^{n} \Delta t\right)\right| \Delta t \leq \frac{v}{2}\left\|\overline{\mathbf{u}}_{h}^{n}\right\|_{1}^{2} \Delta t+\frac{1}{4}\left\|d_{t} \mathbf{u}_{h}^{n}\right\|_{0}^{2} \Delta t^{2}+\left(\frac{2 \gamma_{0}^{2}}{v}+\Delta t\right)\left\|\overline{\mathbf{f}}\left(t_{n}\right)\right\|_{0}^{2} \Delta t \\
& \kappa v^{2}\left|\left(j \bar{T}_{h}^{n}, 2 \overline{\mathbf{u}}_{h}^{n}+d_{t} \mathbf{u}_{h}^{n} \Delta t\right)\right| \Delta t \leq \frac{v}{2}\left\|\overline{\mathbf{u}}_{h}^{n}\right\|_{1}^{2} \Delta t+\frac{1}{4}\left\|d_{t} \mathbf{u}_{h}^{n}\right\|_{0}^{2} \Delta t^{2}+\left(\frac{2 \gamma_{0}^{2}}{v}+\Delta t\right)\left\|\bar{T}_{h}^{n}\right\|_{0}^{2} \Delta t
\end{aligned}
$$

As a consequence, we obtain

$$
\begin{aligned}
& \left(\left\|\mathbf{u}_{h}^{n}\right\|_{0}^{2}+\frac{v}{2}\left\|\mathbf{u}_{h}^{n}\right\|_{1}^{2} \Delta t\right)-\left(\left\|\mathbf{u}_{h}^{n-1}\right\|_{0}^{2}+\frac{v}{2}\left\|\mathbf{u}_{h}^{n-1}\right\|_{1}^{2} \Delta t\right)+v\left\|\overline{\mathbf{u}}_{h}^{n}\right\|_{1}^{2} \Delta t \\
\leq & \left(\frac{2 \gamma_{0}^{2}}{v}+\Delta t\right)\left\|\overline{\mathbf{f}}\left(t_{n}\right)\right\|_{0}^{2} \Delta t+\left(\frac{2 \gamma_{0}^{2}}{v}+\Delta t\right)\left\|\bar{T}_{h}^{n}\right\|_{0}^{2} \Delta t+C G\left(\mathbf{u}_{h}^{n-1}\right)\left\|\mathbf{u}_{h}^{n-1}\right\|_{1}^{2} \Delta t^{2},
\end{aligned}
$$

where

$$
G\left(\mathbf{u}_{h}^{n-1}\right) \Delta t \leq 4^{2} \gamma_{0} \widehat{C}\left\|\mathbf{u}_{h}^{n-1}\right\|_{1}\left\|A_{h} \mathbf{u}_{h}^{n-1}\right\|_{0} \Delta t \leq 4^{2} v^{-3 / 2} \gamma_{0} \widehat{C} C_{1}^{1 / 2} C_{2}^{1 / 2} \Delta t^{1 / 2} \leq v .
$$

Summing above inequalities from $n=3$ to $n=J$, we deduce that

$$
\begin{aligned}
& \left\|\mathbf{u}_{h}^{J}\right\|_{0}^{2}+\frac{v}{2}\left\|\mathbf{u}_{h}^{J}\right\|_{1}^{2} \Delta t+v \Delta t \sum_{n=3}^{J}\left\|\overline{\mathbf{u}}_{h}^{n}\right\|_{1}^{2} \\
& \leq\left\|\mathbf{u}_{h}^{2}\right\|_{0}^{2}+\frac{v}{2}\left\|\mathbf{u}_{h}^{2}\right\|_{1}^{2} \Delta t+\left(\frac{2 \gamma_{0}^{2}}{v}+\Delta t\right) T_{\text {time }}^{\text {final }} \sup _{0 \leq t \leq T_{\text {time }}^{\text {final }}}\|g(t)\|_{0}^{2}
\end{aligned}
$$




$$
+\left(\frac{2 \gamma_{0}^{2}}{v}+\Delta t\right) \sum_{n=3}^{J}\left\|\bar{T}_{h}^{n}\right\|_{0}^{2} \Delta t+C_{1} \Delta t .
$$

Using Theorem 3.1, (3.9) in (3.12)-(3.13) to obtain (3.10a) with $m=J$.

Secondly, choosing $\psi_{h}=2 A_{h} \bar{T}_{h}^{n} \Delta t \in W_{h}, \mathbf{v}_{h}=0, q_{h}=0$ in (3.2), we get

$$
\left(d_{t} T_{h}^{n}, 2 A_{h} \bar{T}_{h}^{n} \Delta t\right)+\tilde{a}\left(\bar{T}_{h}^{n}, 2 A_{h} \bar{T}_{h}^{n} \Delta t\right)+\tilde{b}\left(\mathbf{u}_{h}^{n-1}, T_{h}^{n-1}, 2 A_{h} \bar{T}_{h}^{n} \Delta t\right)=\left(\bar{g}\left(t_{n}\right), 2 A_{h} \bar{T}_{h}^{n} \Delta t\right) .
$$

Thanks to Lemma 2.2 and the Cauchy inequality, one yields

$$
\begin{aligned}
& 2\left|\tilde{b}\left(\mathbf{u}_{h}^{n-1}, T_{h}^{n-1}, A_{h} \bar{T}_{h}^{n}\right)\right| \Delta t \leq \frac{1}{4} G^{\frac{1}{2}}\left(\mathbf{u}_{h}^{n-1}\right)\left\|T_{h}^{n-1}\right\|_{1}\left\|A_{h} \bar{T}_{h}^{n}\right\|_{0} \Delta t \\
& \leq \frac{\operatorname{Pr}^{-1} v}{2}\left\|A_{h} \bar{T}_{h}^{n}\right\|_{0}^{2} \Delta t+\frac{1}{32 \operatorname{Pr}^{-1} v} G\left(\mathbf{u}_{h}^{n-1}\right)\left\|T_{h}^{n-1}\right\|_{1}^{2} \Delta t, \\
& 2\left|\left(\bar{g}\left(t_{n}\right), A_{h} \bar{T}_{h}^{n} \Delta t\right)\right| \leq \frac{\operatorname{Pr}^{-1} v}{2}\left\|A_{h} \bar{T}_{h}^{n}\right\|_{0}^{2} \Delta t+\frac{1}{2 \operatorname{Pr}^{-1} v}\left\|\bar{g}\left(t_{n}\right)\right\|_{0}^{2} \Delta t .
\end{aligned}
$$

Combining above inequalities with (3.15), we obtain

$$
\begin{aligned}
& \left\|T_{h}^{n}\right\|_{1}^{2}-\left\|T_{h}^{n-1}\right\|_{1}^{2}+\operatorname{Pr}^{-1} v\left\|A_{h} \bar{T}_{h}^{n}\right\|_{0}^{2} \Delta t \\
\leq & \frac{1}{32 P r^{-1} v} G\left(\mathbf{u}_{h}^{n-1}\right)\left\|T_{h}^{n-1}\right\|_{1}^{2} \Delta t+\frac{1}{2 P r^{-1} v}\left\|\bar{g}\left(t_{n}\right)\right\|_{0}^{2} \Delta t .
\end{aligned}
$$

Summing above inequality from $n=3$ to $n=J$ and using (3.12), we deduce that

$$
\begin{aligned}
& \left\|T_{h}^{J}\right\|_{1}^{2}+\operatorname{Pr}^{-1} v \Delta t \sum_{n=3}^{J}\left\|A_{h} \bar{T}_{h}^{n}\right\|_{0}^{2} \\
\leq & \frac{1}{32} C_{1} \operatorname{Prv}^{-1}+\frac{1}{\operatorname{Pr}^{-1} v} T_{\text {time }}^{\text {final }} \sup _{0 \leq t \leq T_{\text {time }}^{\text {final }}}\|g(t)\|_{0}^{2}+\left\|T_{h}^{2}\right\|_{1}^{2} .
\end{aligned}
$$

Thirdly, choosing $\mathbf{v}_{h}=2 A_{h} \overline{\mathbf{u}}_{h}^{n} \Delta t \in V_{h}, q_{h}=0, \psi_{h}=0$ in (3.2), we get

$$
\begin{aligned}
& \left(d_{t} \mathbf{u}_{h}^{n}, 2 A_{h} \overline{\mathbf{u}}_{h}^{n} \Delta t\right)+a\left(\overline{\mathbf{u}}_{h}^{n}, 2 A_{h} \overline{\mathbf{u}}_{h}^{n} \Delta t\right)+b\left(\mathbf{u}_{h}^{n-1}, \mathbf{u}_{h}^{n-1}, 2 A_{h} \overline{\mathbf{u}}_{h}^{n} \Delta t\right) \\
= & \left(\overline{\mathbf{f}}\left(t_{n}\right), 2 A_{h} \overline{\mathbf{u}}_{h}^{n} \Delta t\right)-\kappa v^{2}\left(j \bar{T}_{h}^{n}, 2 A_{h} \overline{\mathbf{u}}_{h}^{n} \Delta t\right) .
\end{aligned}
$$

With the help of Lemma 2.2 and the Cauchy inequality, one yields

$$
\begin{aligned}
& 2\left|b\left(\mathbf{u}_{h}^{n-1}, \mathbf{u}_{h}^{n-1}, A_{h} \overline{\mathbf{u}}_{h}^{n}\right)\right| \Delta t \leq \frac{1}{4} G^{\frac{1}{2}}\left(\mathbf{u}_{h}^{n-1}\right)\left\|\mathbf{u}_{h}^{n-1}\right\|_{1}\left\|A_{h} \overline{\mathbf{u}}_{h}^{n}\right\|_{0} \Delta t \\
& \leq \frac{v}{2}\left\|A_{h} \overline{\mathbf{u}}_{h}^{n}\right\|_{0}^{2} \Delta t+\frac{1}{32 v} G\left(\mathbf{u}_{h}^{n-1}\right)\left\|\mathbf{u}_{h}^{n-1}\right\|_{1}^{2} \Delta t, \\
& 2\left|\left(\overline{\mathbf{f}}\left(t_{h}\right), A_{h} \overline{\mathbf{u}}_{h}^{n} \Delta t\right)\right| \leq \frac{v}{4}\left\|A_{h} \overline{\mathbf{u}}_{h}^{n}\right\|_{0}^{2} \Delta t+\frac{4}{v}\left\|\overline{\mathbf{f}}\left(t_{h}\right)\right\|_{0}^{2} \Delta t, \\
& 2\left|-\kappa v^{2}\left(j \bar{T}_{h}^{n}, A_{h} \overline{\mathbf{u}}_{h}^{n}\right)\right| \Delta t \leq \frac{v}{4}\left\|A_{h} \overline{\mathbf{u}}_{h}^{n}\right\|_{0}^{2} \Delta t+4 \kappa^{2} v^{3}\left\|\bar{T}_{h}^{n}\right\|_{0}^{2} \Delta t .
\end{aligned}
$$


As a consequence, we obtain

$$
\begin{aligned}
& \left\|\mathbf{u}_{h}^{n}\right\|_{1}^{2}-\left\|\mathbf{u}_{h}^{n-1}\right\|_{1}^{2}+v\left\|A_{h} \overline{\mathbf{u}}_{h}^{n}\right\|_{0}^{2} \Delta t \\
\leq & \frac{1}{32 v} G\left(\mathbf{u}_{h}^{n-1}\right)\left\|\mathbf{u}_{h}^{n-1}\right\|_{1}^{2} \Delta t+\frac{4}{v}\left\|\overline{\mathbf{f}}\left(t_{n}\right)\right\|_{0}^{2} \Delta t+4 \kappa^{2} v^{3}\left\|\bar{T}_{h}^{n}\right\|_{0}^{2} \Delta t .
\end{aligned}
$$

Summing above inequality from $n=3$ to $n=J$ and using (3.13), we deduce

$$
\begin{aligned}
& \left\|\mathbf{u}_{h}^{J}\right\|_{1}^{2}+v \Delta t \sum_{n=3}^{J}\left\|A_{h} \overline{\mathbf{u}}_{h}^{n}\right\|_{0}^{2} \\
\leq & \left\|\mathbf{u}_{h}^{2}\right\|_{1}^{2}+\frac{C_{1}}{32 v}+\frac{4}{v} T_{\text {time }}^{\text {final }} \sup _{\substack{0 \leq t \leq T_{\text {time }}^{\text {final }} \\
\|}}\|f(t)\|_{0}^{2}+4 \kappa^{2} v^{3} \sum_{n=3}^{J}\left\|\bar{T}_{h}^{n}\right\|_{0}^{2} \Delta t .
\end{aligned}
$$

Thanks to (3.9) and (3.10a), using Theorem 3.1 to (3.16) and (3.17), we obtain

$$
\left\|\left(\mathbf{u}_{h}^{J}, T_{h}^{J}\right)\right\|_{1}^{2}+\min \left\{v, \operatorname{Pr}^{-1} v\right\} \Delta t \sum_{n=3}^{J}\left\|\left(A_{h} \overline{\mathbf{u}}_{h}^{n}, A_{h} \bar{T}_{h}^{n}\right)\right\|_{0}^{2} \leq C, \quad \forall 1 \leq J \leq N .
$$

From (3.2), Lemma 2.2 and the assumption (A3), we deduce that

$$
\begin{aligned}
& \left(\left\|p_{h}^{n}\right\|_{0}^{2}+\left\|d_{t} \mathbf{u}_{h}^{n}\right\|_{0}^{2}\right) \Delta t \\
& \leq v^{2}\left\|A_{h} \overline{\mathbf{u}}_{h}^{n}\right\|_{0}^{2} \Delta t+\widehat{C} \gamma_{0}^{2}\left\|\mathbf{u}_{h}^{n-1}\right\|_{1}^{2}\left\|A_{h} \mathbf{u}_{h}^{n-1}\right\|_{0}^{2} \Delta t+\kappa^{2} v^{4}\left\|\bar{T}_{h}^{n}\right\|_{0}^{2} \Delta t+\left\|\overline{\mathbf{f}}\left(t_{n}\right)\right\|_{0}^{2} \Delta t, \\
& \left\|d_{t} T_{h}^{n}\right\|_{0}^{2} \Delta t \leq \operatorname{Pr}^{-2} v^{2}\left\|A_{h} \bar{T}_{h}^{n}\right\|_{0}^{2} \Delta t+\widetilde{C} \gamma_{0}^{2}\left\|\mathbf{u}_{h}^{n-1}\right\|_{1}^{2}\left\|A_{h} T_{h}^{n-1}\right\|_{0}^{2} \Delta t+\left\|\bar{g}\left(t_{n}\right)\right\|_{0}^{2} \Delta t .
\end{aligned}
$$

Summing (3.19a) and (3.19b) from $n=3$ to $J$, using Lemma 2.1, (3.10a) and (3.18) and the induction assumption, we obtain (3.10b) with $m=J$.

Finally, choosing $\psi_{h}=2 A_{h} d_{t} T_{h}^{n} \Delta t^{2} \in W_{h}, \mathbf{v}_{h}=0, q_{h}=0$ in (3.2) and noting the fact that $\phi_{h}^{n}-\phi_{h}^{n-1}=2\left(\bar{\phi}_{h}^{n}-\phi_{h}^{n-1}\right), \phi$ takes $\mathbf{u}$ and $T$, we get

$$
\begin{aligned}
& \left(d_{t} T_{h}^{n}, 2 A_{h} d_{t} T_{h}^{n} \Delta t^{2}\right)+\tilde{a}\left(\bar{T}_{h}^{n}, 2 A_{h} d_{t} T_{h}^{n} \Delta t^{2}\right)+\tilde{b}\left(\mathbf{u}_{h}^{n-1}, T_{h}^{n-1}, 2 A_{h} d_{t} T_{h}^{n} \Delta t^{2}\right) \\
= & \left(\bar{g}\left(t_{n}\right), 2 A_{h} d_{t} T_{h}^{n} \Delta t^{2}\right) .
\end{aligned}
$$

Thanks to Lemma 2.2 and the Cauchy inequality, one finds

$$
\begin{aligned}
& 2\left|\tilde{b}\left(\mathbf{u}_{h}^{n-1}, T_{h}^{n-1}, A_{h} d_{t} T_{h}^{n} \Delta t^{2}\right)\right| \leq \widetilde{C}\left\|A_{h} \mathbf{u}_{h}^{n-1}\right\|_{0}\left\|A_{h} T_{h}^{n-1}\right\|_{0}\left\|d_{t} T_{h}^{n}\right\|_{1} \Delta t^{2} \\
& \leq \frac{1}{2}\left\|d_{t} T_{h}^{n}\right\|_{1}^{2} \Delta t^{2}+2 \widetilde{C} \gamma_{0}^{2}\left\|A_{h} \mathbf{u}_{h}^{n-1}\right\|_{0}^{2}\left\|A_{h} T_{h}^{n-1}\right\|_{0}^{2} \Delta t^{2}, \\
& \left|\left(\bar{g}\left(t_{n}\right), 2 A_{h} d_{t} T_{h}^{n} \Delta t^{2}\right)\right| \leq \frac{1}{2}\left\|d_{t} T_{h}^{n}\right\|_{1}^{2} \Delta t^{2}+2\left\|\bar{g}\left(t_{n}\right)\right\|_{1}^{2} \Delta t^{2} .
\end{aligned}
$$

Combining above inequalities with (3.20), we get

$$
\begin{aligned}
& \operatorname{Pr}^{-1} v\left(\left\|A_{h} T_{h}^{n}\right\|_{0}^{2} \Delta t-\left\|A_{h} T_{h}^{n-1}\right\|_{0}^{2} \Delta t\right)+\left\|d_{t} T_{h}^{n}\right\|_{1}^{2} \Delta t^{2} \\
\leq & 2 \widetilde{C} \gamma_{0}^{2}\left\|A_{h} \mathbf{u}_{h}^{n-1}\right\|_{0}^{2}\left\|A_{h} T_{h}^{n-1}\right\|_{0}^{2} \Delta t^{2}+2\left\|\bar{g}\left(t_{h}\right)\right\|_{1}^{2} \Delta t^{2} .
\end{aligned}
$$


Summing above inequality from $n=3$ to $J$ and using (3.10b), we deduce that

$$
\begin{aligned}
& \operatorname{Pr}^{-1} v\left\|A_{h} T_{h}^{J}\right\|_{0}^{2} \Delta t+\Delta t^{2} \sum_{n=3}^{J}\left\|d_{t} T_{h}^{n}\right\|_{1}^{2} \\
\leq & 2 T_{\text {time }}^{\text {final }} \sup _{0 \leq t \leq T_{\text {time }}^{\text {final }}}\|g(t)\|_{1}^{2} \Delta t^{2}+\operatorname{Pr}^{-1} v\left\|A_{h} T_{h}^{2}\right\|_{0}^{2} \Delta t+C_{2}^{2} \operatorname{Pr}^{4} v^{-4} .
\end{aligned}
$$

Choosing $\mathbf{v}_{h}=2 A_{h} d_{t} \mathbf{u}_{h}^{n} \Delta t^{2} \in V_{h}, \psi_{h}=0, q_{h}=0$ in (3.2), we obtain

$$
\begin{aligned}
& \left(d_{t} \mathbf{u}_{h}^{n}, 2 A_{h} d_{t} \mathbf{u}_{h}^{n} \Delta t^{2}\right)+a\left(\overline{\mathbf{u}}_{h}^{n}, 2 A_{h} d_{t} \mathbf{u}_{h}^{n} \Delta t^{2}\right)+b\left(\mathbf{u}_{h}^{n-1}, \mathbf{u}_{h}^{n-1}, 2 A_{h} d_{t} \mathbf{u}_{h}^{n} \Delta t^{2}\right) \\
= & \left(\overline{\mathbf{f}}\left(t_{n}\right), 2 A_{h} d_{t} \mathbf{u}_{h}^{n} \Delta t^{2}\right)-\kappa v^{2}\left(j \bar{T}_{h}^{n}, 2 A_{h} d_{t} \mathbf{u}_{h}^{n} \Delta t^{2}\right) .
\end{aligned}
$$

Applying Lemma 2.2 and the Cauchy inequality, one yields

$$
\begin{aligned}
& 2\left|b\left(\mathbf{u}_{h}^{n-1}, \mathbf{u}_{h}^{n-1}, A_{h} d_{t} \mathbf{u}_{h}^{n}\right)\right| \Delta t^{2} \leq \frac{1}{2}\left\|d_{t} \mathbf{u}_{h}^{n}\right\|_{1}^{2} \Delta t^{2}+2 \widehat{C} \gamma_{0}^{2}\left\|A_{h} \mathbf{u}_{h}^{n-1}\right\|_{0}^{4} \Delta t^{2}, \\
& 2\left|\left(\overline{\mathbf{f}}\left(t_{n}\right), A_{h} d_{t} \mathbf{u}_{h}^{n}\right)\right| \Delta t^{2} \leq \frac{1}{4}\left\|d_{t} \mathbf{u}_{h}^{n}\right\|_{1}^{2} \Delta t^{2}+4\left\|\bar{f}\left(t_{n}\right)\right\|_{1}^{2} \Delta t^{2}, \\
& 2\left|-\kappa v^{2}\left(j \bar{T}_{h}^{n}, A_{h} d_{t} \mathbf{u}_{h}^{n}\right)\right| \Delta t^{2} \leq \frac{1}{4}\left\|d_{t} \mathbf{u}_{h}^{n}\right\|_{1}^{2} \Delta t^{2}+4 \kappa^{2} v^{4}\left\|\bar{T}_{h}^{n}\right\|_{1}^{2} \Delta t^{2} .
\end{aligned}
$$

Combing above inequalities with (3.22), we get

$$
\begin{aligned}
& v\left(\left\|A_{h} \mathbf{u}_{h}^{n}\right\|_{0}^{2}-\left\|A_{h} \mathbf{u}_{h}^{n-1}\right\|_{0}^{2}\right) \Delta t+\left\|d_{t} \mathbf{u}_{h}^{n}\right\|_{1}^{2} \Delta t^{2} \\
\leq & 2 \widehat{C} \gamma_{0}^{2}\left\|A_{h} \mathbf{u}_{h}^{n-1}\right\|_{0}^{4} \Delta t^{2}+4\left\|\bar{f}\left(t_{n}\right)\right\|_{1}^{2} \Delta t^{2}+4 \kappa^{2} v^{4}\left\|\bar{T}_{h}^{n}\right\|_{1}^{2} \Delta t^{2} .
\end{aligned}
$$

Summing above inequality from $n=3$ to $J$ and using (3.9), we arrive at

$$
\begin{aligned}
& v\left\|A_{h} \mathbf{u}_{h}^{J}\right\|_{0}^{2} \Delta t+\Delta t^{2} \sum_{n=3}^{J}\left\|d_{t} \mathbf{u}_{h}^{n}\right\|_{1}^{2} \\
& \leq v\left\|A_{h} \mathbf{u}_{h}^{2}\right\|_{0}^{2} \Delta t+4 T_{\text {time }}^{\text {final }} \sup _{0 \leq t \leq T_{\text {time }}^{\text {final }}}\|\mathbf{f}(t)\|_{1}^{2} \Delta t^{2}+4 \kappa^{2} v^{4} \sum_{n=3}^{J}\left\|\bar{T}_{h}^{n}\right\|_{1}^{2} \Delta t^{2}+\frac{C_{2}^{2}}{v^{2}} .
\end{aligned}
$$

Thanks to (3.9), (3.10b), using Lemma 2.1 to (3.21) and (3.23), we complete the proof of (3.10c) with $m=J$.

We end this section by recalling the following priori estimates provided in [31].

Theorem 3.3. Suppose that the assumptions (A1)-(A3) and (3.9) hold, it holds

$$
\begin{aligned}
& \left\|\left(e_{u}^{1}, e_{T}^{1}\right)\right\|_{\alpha}^{2}+\min \left\{v, P^{-1} v\right\}\left\|\left(e_{u}^{1}, e_{T}^{1}\right)\right\|_{\alpha+1}^{2} \Delta t \leq C \Delta t^{1-\alpha}, \quad \alpha=-2,-1,0,1, \\
& \left\|e_{p}^{1}\right\|_{0}^{2} \leq C \Delta t^{-1},
\end{aligned}
$$

where

$$
e_{\phi}^{0}=0, \quad e_{\phi}^{n}=\phi_{h}\left(t_{n}\right)-\phi_{h}^{n}, \quad \phi \text { takes } \boldsymbol{u} \text { or } T, \quad e_{p}^{n}=\frac{1}{\Delta t} \int_{t_{n-1}}^{t_{n}} p_{h}(s) d s-p_{h}^{n}, \quad n=1, \cdots, N .
$$




\section{Error estimates}

In this section, we present the errors of the numerical solutions $e_{\mathbf{u}}^{n}=\mathbf{u}_{h}\left(t_{n}\right)-\mathbf{u}_{h}^{n}$ and $e_{T}^{n}=$ $T_{h}\left(t_{n}\right)-T_{h}^{n}$ in $H^{1}$-and $L^{2}$-norms and the error

$$
e_{p}^{n}=\frac{1}{\Delta t} \int_{t_{n-1}}^{t_{n}} p_{h}(s) d s-p_{h}^{n}
$$

in $L^{2}$-norm with nonsmooth initial data. For all $t \in\left[0, T_{\text {time }}^{\text {final }}\right]$ and $1 \leq n \leq N$, integrate (2.3) from $t_{n-1}$ to $t_{n}$, we obtain

$$
\left\{\begin{array}{l}
\left(d_{t} \mathbf{u}_{h}\left(t_{n}\right), \mathbf{v}_{h}\right)+\frac{1}{\Delta t} \int_{t_{n-1}}^{t_{n}} a\left(\mathbf{u}_{h}(s), \mathbf{v}_{h}\right) d s-\frac{1}{\Delta t} \int_{t_{n-1}}^{t_{n}} d\left(\mathbf{v}_{h}, p_{h}(s)\right) d s \\
\quad+\frac{1}{\Delta t} \int_{t_{n-1}}^{t_{n}} b\left(\mathbf{u}_{h}(s), \mathbf{u}_{h}(s), \mathbf{v}_{h}\right) d s=\frac{1}{\Delta t} \int_{t_{n-1}}^{t_{n}}\left(\mathbf{f}(s), \mathbf{v}_{h}\right) d s-\frac{\kappa v^{2}}{\Delta t} \int_{t_{n-1}}^{t_{n}}\left(T_{h}(s), \mathbf{v}_{h}\right) d s \\
d\left(\overline{\mathbf{u}}_{h}\left(t_{n}\right), q_{h}\right)=0, \\
\left(d_{t} T_{h}\left(t_{n}\right), \psi_{h}\right)+\frac{1}{\Delta t} \int_{t_{n-1}}^{t_{n}} \tilde{a}\left(T_{h}(s), \psi_{h}\right) d s+\frac{1}{\Delta t} \int_{t_{n-1}}^{t_{n}} \tilde{b}\left(\mathbf{u}_{h}(s), T_{h}(s), \psi_{h}\right) d s \\
=\frac{1}{\Delta t} \int_{t_{n-1}}^{t_{n}}\left(g(s), \psi_{h}\right) d s .
\end{array}\right.
$$

For all $\phi \in L^{2}\left(t_{n-1}, t_{n} ; H^{2}\left(t_{n-1}, t_{n}\right)\right)$, using the integral formula

$$
\bar{\phi}\left(t_{n}\right)-\frac{1}{\Delta t} \int_{t_{n-1}}^{t_{n}} \phi(s) d s=\frac{1}{2 \Delta t} \int_{t_{n-1}}^{t_{n}}\left(s-t_{n-1}\right)\left(t_{n}-s\right) \phi_{s s}(s) d s .
$$

Subtracting (3.2) from (4.1), we get

$$
\left\{\begin{array}{l}
\left(d_{t} e_{\mathbf{u}}^{n}, \mathbf{v}_{h}\right)+a\left(\bar{e}_{\mathbf{u}}^{n}, \mathbf{v}_{h}\right)-d\left(\mathbf{v}_{h}, e_{p}^{n}\right)+b\left(\mathbf{u}_{h}^{n-1}, e_{\mathbf{u}}^{n-1}, \mathbf{v}_{h}\right) \\
\quad+b\left(e_{\mathbf{u}}^{n-1}, \mathbf{u}_{h}\left(t_{n-1}\right), \mathbf{v}_{h}\right)=\left(e_{n}, \mathbf{v}_{h}\right) \\
d\left(\bar{e}_{\mathbf{u}}^{n}, q_{h}\right)=0 \\
\left(d_{t} e_{T}^{n}, \psi_{h}\right)+\tilde{a}\left(\bar{e}_{T}^{n}, \psi_{h}\right)+\tilde{b}\left(\mathbf{u}_{h}^{n-1}, e_{T}^{n-1}, \psi_{h}\right) \\
\quad+\tilde{b}\left(e_{\mathbf{u}}^{n-1}, T_{h}\left(t_{n-1}\right), \psi_{h}\right)=\left(e_{n}^{\prime}, \psi_{h}\right)
\end{array}\right.
$$

where

$$
\begin{aligned}
\left(e_{n}, \mathbf{v}_{h}\right)= & \frac{1}{2 \Delta t} \int_{t_{n-1}}^{t_{n}}\left(s-t_{n-1}\right)\left(t_{n}-s\right)\left(\mathbf{f}_{s s}, \mathbf{v}_{h}\right) d s-\frac{\kappa v^{2}}{2 \Delta t} \int_{t_{n-1}}^{t_{n}}\left(s-t_{n-1}\right)\left(t_{n}-s\right)\left(j T_{h s s}, \mathbf{v}_{h}\right) d s \\
& +\frac{1}{\Delta t} \int_{t_{n-1}}^{t_{n}}\left(s-t_{n-1}\right)\left(t_{n}-s\right) a\left(\mathbf{u}_{h s s}, \mathbf{v}_{h}\right) d s+\frac{1}{2 \Delta t} \int_{t_{n-1}}^{t_{n}}\left(s-t_{n-1}\right) b_{s}\left(\mathbf{u}_{h}(s), \mathbf{u}_{h}(s), \mathbf{v}_{h}\right) d s \\
& +\frac{1}{2 \Delta t} \int_{t_{n-1}}^{t_{n}}\left(t_{n}-s\right) b_{s}\left(\mathbf{u}_{h}(s), \mathbf{u}_{h}(s), \mathbf{v}_{h}\right) d s-\frac{2 \kappa v^{2}}{\Delta t} \int_{t_{n-1}}^{t_{n}}\left(j T_{h}(s), \mathbf{v}_{h}\right) d s \\
& +\frac{1}{2 \Delta t} \int_{t_{n-1}}^{t_{n}}\left(s-t_{n-1}\right)\left(t_{n}-s\right) b_{s s}\left(\mathbf{u}_{h}(s), \mathbf{u}_{h}(s), \mathbf{v}_{h}\right) d s,
\end{aligned}
$$




$$
\begin{aligned}
\left(e_{n}^{\prime}, \psi_{h}\right)= & \frac{1}{2 \Delta t} \int_{t_{n-1}}^{t_{n}}\left(s-t_{n-1}\right)\left(t_{n}-s\right)\left(g_{s s}, \psi_{h}\right) d s+\frac{1}{\Delta t} \int_{t_{n-1}}^{t_{n}}\left(s-t_{n-1}\right)\left(t_{n}-s\right) \tilde{a}\left(T_{h s s}, \psi_{h}\right) d s \\
& +\frac{1}{2 \Delta t} \int_{t_{n-1}}^{t_{n}}\left(s-t_{n-1}\right) \tilde{b}_{s}\left(\mathbf{u}_{h}(s), T_{h}(s), \psi_{h}\right) d s+\frac{1}{2 \Delta t} \int_{t_{n-1}}^{t_{n}}\left(t_{n}-s\right) \tilde{b}_{s}\left(\mathbf{u}_{h}(s), T_{h}(s), \psi_{h}\right) d s \\
& +\frac{1}{2 \Delta t} \int_{t_{n-1}}^{t_{n}}\left(s-t_{n-1}\right)\left(t_{n}-s\right) \tilde{b}_{s s}\left(\mathbf{u}_{h}(s), T_{h}(s), \psi_{h}\right) d s,
\end{aligned}
$$

furthermore, we know that

$$
\begin{aligned}
& b_{t t}\left(\mathbf{u}_{h}(t), \mathbf{u}_{h}(t), \mathbf{v}_{h}\right)=b\left(\mathbf{u}_{h t t}(t), \mathbf{u}_{h}(t), \mathbf{v}_{h}\right)+b\left(\mathbf{u}_{h}(t), \mathbf{u}_{h t t}(t), \mathbf{v}_{h}\right)+2 b\left(\mathbf{u}_{h t}, \mathbf{u}_{h t}, \mathbf{v}_{h}\right), \\
& \tilde{b}_{t t}\left(\mathbf{u}_{h}(t), T_{h}(t), \psi_{h}\right)=\tilde{b}\left(\mathbf{u}_{h t t}(t), T_{h}(t), \psi_{h}\right)+\tilde{b}\left(\mathbf{u}_{h}(t), T_{h t t}(t), \psi_{h}\right)+2 \tilde{b}\left(\mathbf{u}_{h t}, T_{h t}, \psi_{h}\right) .
\end{aligned}
$$

Lemma 4.1 ([31]). Suppose that the assumptions (A1)-(A3) and (3.9) hold, for all $3 \leq m \leq N$, the errors $e_{n}$ and $e_{n}^{\prime}$ satisfy

$$
\begin{array}{ll}
\Delta t \sum_{n=3}^{m} \sigma^{i}\left(t_{n}\right)\left\|A_{h}^{-1} P_{h} e_{n}, A_{h}^{-1} P_{h} e_{n}^{\prime}\right\|_{0}^{2} \leq C \Delta t^{2+i}, & i=0,1,2, \\
\Delta t \sum_{n=3}^{m} \sigma^{i}\left(t_{n}\right)\left\|A_{h}^{-1 / 2} P_{h} e_{n}, A_{h}^{-1 / 2} P_{h} e_{n}^{\prime}\right\|_{0}^{2} \leq C \Delta t^{1+i}, & i=0,1,2,3, \\
\Delta t \sum_{n=3}^{m} \sigma^{i}\left(t_{n}\right)\left\|P_{h} e_{n}, P_{h} e_{n}^{\prime}\right\|_{0}^{2} \leq C \Delta t^{i}, & i=0,1,2,3,4, \\
\Delta t \sum_{n=3}^{m} \sigma^{i}\left(t_{n}\right)\left\|A_{h}^{1 / 2} P_{h} e_{n}, A_{h}^{1 / 2} P_{h} e_{n}^{\prime}\right\|_{0}^{2} \leq C \Delta t^{i-1,} & i=0,1,2,3,4,
\end{array}
$$

where

$$
\sigma\left(t_{n}\right) \leq \sigma\left(t_{n-1}\right)+\Delta t \leq 2 \sigma(t), \quad \sigma\left(t_{n-1}\right) \leq \sigma(t), \quad t \in\left[t_{n-1}, t_{n}\right], \quad 2 \leq n \leq N .
$$

Theorem 4.1. Under the conditions of (A1)-(A3) and (3.9), it holds

$$
\begin{aligned}
& \left\|e_{u}^{m}, e_{T}^{m}\right\|_{\alpha}^{2}+\min \left\{v, \operatorname{Pr}^{-1} v\right\}\left\|e_{u}^{m}, e_{T}^{m}\right\|_{\alpha+1}^{2} \Delta t+\Delta t \sum_{n=1}^{m}\left(\left\|d_{t} e_{u}^{n}, d_{t} e_{T}^{n}\right\|_{\alpha}^{2} \Delta t\right. \\
& \left.\quad+\min \left\{v, \operatorname{Pr}^{-1} v\right\}\left\|e_{u}^{n}, \bar{e}_{T}^{n}\right\|_{\alpha+1}^{2}\right) \leq C \Delta t^{1-\alpha}, \quad \forall 1 \leq m \leq N, \quad \alpha=-1,0,1, \\
& \left\|e_{u}^{m}, e_{T}^{m}\right\|_{-2}^{2}+\min \left\{v, \operatorname{Pr}^{-1} v\right\}\left\|e_{u}^{m}, e_{T}^{m}\right\|_{-1}^{2} \Delta t+\Delta t \sum_{n=1}^{m}\left(\left\|d_{t} e_{u}^{n}, d_{t} e_{T}^{n}\right\|_{-2}^{2} \Delta t\right. \\
& \left.\quad+\min \left\{v, \operatorname{Pr}^{-1} v\right\}\left\|\bar{e}_{u}^{n}, \bar{e}_{T}^{n}\right\|_{-1}^{2}\right) \leq C \Delta t^{3}, \quad m=1,2 .
\end{aligned}
$$

Proof. From Theorem 3.3, we know that (4.3b) and (4.3a) hold with $m=1$.

Taking $\mathbf{v}_{h}=2 A_{h}^{\alpha} e_{\mathbf{u}}^{n} \Delta t \in V_{h}, \psi_{h}=0$ with $\alpha=-1,0,1$ in (4.2), we get

$$
\begin{aligned}
& \left(d_{t} e_{\mathbf{u}}^{n}, 2 A_{h}^{\alpha} e_{\mathbf{u}}^{n} \Delta t\right)+a\left(\bar{e}_{\mathbf{u}}^{n}, 2 A_{h}^{\alpha} e_{\mathbf{u}}^{n} \Delta t\right)+b\left(\mathbf{u}_{h}^{n-1}, e_{\mathbf{u}}^{n-1}, 2 A_{h}^{\alpha} e_{\mathbf{u}}^{n} \Delta t\right) \\
& \quad+b\left(e_{\mathbf{u}}^{n-1}, \mathbf{u}_{h}\left(t_{n-1}\right), 2 A_{h}^{\alpha} e_{\mathbf{u}}^{n} \Delta t\right)=\left(e_{n}, 2 A_{h}^{\alpha} e_{\mathbf{u}}^{n} \Delta t\right) .
\end{aligned}
$$




\section{Using}

$$
e_{\phi}^{n}=\bar{e}_{\phi}^{n}+\frac{1}{2} d_{t} e_{\phi}^{n} \Delta t \quad \text { and } \quad e_{\phi}^{n-1}=\bar{e}_{\phi}^{n}-\frac{1}{2} d_{t} e_{\phi}^{n} \Delta t
$$

where $\phi$ can take $\mathbf{u}$ and $T$, we obtain

$$
\begin{aligned}
& \left\|e_{\mathbf{u}}^{n}\right\|_{\alpha}^{2}-\left\|e_{\mathbf{u}}^{n-1}\right\|_{\alpha}^{2}+\left\|d_{t} e_{\mathbf{u}}^{n}\right\|_{\alpha}^{2} \Delta t^{2}+\frac{v}{2}\left(\left\|e_{\mathbf{u}}^{n}\right\|_{\alpha+1}^{2}-\left\|e_{\mathbf{u}}^{n-1}\right\|_{\alpha+1}^{2}+4\left\|\bar{e}_{\mathbf{u}}^{n}\right\|_{\alpha+1}^{2}\right) \Delta t \\
& \quad+2 b\left(e_{\mathbf{u}}^{n-1}, \mathbf{u}_{h}\left(t_{n-1}\right), A_{h}^{\alpha} e_{\mathbf{u}}^{n}\right) \Delta t+2 b\left(\mathbf{u}_{h}\left(t_{n-1}\right), e_{\mathbf{u}}^{n-1}, A_{h}^{\alpha} e_{\mathbf{u}}^{n}\right) \Delta t-2 b\left(\bar{e}_{\mathbf{u}}^{n-1}, \bar{e}_{\mathbf{u}}^{n-1}, A_{h}^{\alpha} e_{\mathbf{u}}^{n}\right) \Delta t \\
& \quad-b\left(d_{t} e_{\mathbf{u}}^{n-1}, \bar{e}_{\mathbf{u}}^{n-1}, A_{h}^{\alpha} e_{\mathbf{u}}^{n}\right) \Delta t^{2}-b\left(\bar{e}_{\mathbf{u}}^{n-1}, d_{t} e_{\mathbf{u}}^{n-1}, A_{h}^{\alpha} e_{\mathbf{u}}^{n}\right) \Delta t^{2}-\frac{1}{2} b\left(d_{t} e_{\mathbf{u}}^{n-1}, d_{t} e_{\mathbf{u}}^{n-1}, A_{h}^{\alpha} e_{\mathbf{u}}^{n}\right) \Delta t^{3} \\
& \leq \frac{v}{8}\left\|\bar{e}_{\mathbf{u}}^{n}\right\|_{\alpha+1}^{2} \Delta t+\frac{1}{16}\left\|d_{t} e_{\mathbf{u}}^{n}\right\|_{\alpha}^{2} \Delta t^{2}+8 v^{-1}\left\|A_{h}^{\frac{\alpha-1}{2}} P_{h} e_{n}\right\|_{0}^{2} \Delta t+16\left\|A_{h}^{\frac{\alpha}{2}} e_{n}\right\|_{0}^{2} \Delta t^{2} .
\end{aligned}
$$

Thanks to Lemma 2.2 and (2.4), we have

$$
\begin{aligned}
& 2\left|b\left(e_{\mathbf{u}}^{n-1}, \mathbf{u}_{h}\left(t_{n-1}\right), A_{h}^{\alpha} e_{\mathbf{u}}^{n}\right)\right| \Delta t+2\left|b\left(\mathbf{u}_{h}\left(t_{n-1}\right), e_{\mathbf{u}}^{n-1}, A_{h}^{\alpha} e_{\mathbf{u}}^{n}\right)\right| \Delta t \\
& =2\left|b\left(e_{\mathbf{u}}^{n-1}, \mathbf{u}_{h}\left(t_{n-1}\right), A_{h}^{\alpha} \bar{e}_{\mathbf{u}}^{n}+\frac{1}{2} A_{h}^{\alpha} d_{t} e_{\mathbf{u}}^{n} \Delta t\right)\right| \Delta t+2\left|b\left(\mathbf{u}_{h}\left(t_{n-1}\right), e_{\mathbf{u}}^{n-1}, A_{h}^{\alpha} \bar{e}_{\mathbf{u}}^{n}+\frac{1}{2} A_{h}^{\alpha} d_{t} e_{\mathbf{u}}^{n} \Delta t\right)\right| \Delta t \\
& \leq 2 \widehat{C} \gamma_{0}\left\|e_{\mathbf{u}}^{n-1}\right\|_{\alpha}\left\|A_{h} \mathbf{u}_{h}\left(t_{n-1}\right)\right\|_{0}\left\|\bar{e}_{\mathbf{u}}^{n}\right\|_{\alpha+1} \Delta t+\widehat{C} \gamma_{0}\left\|e_{\mathbf{u}}^{n-1}\right\|_{\alpha+1}\left\|A_{h} \mathbf{u}_{h}\left(t_{n-1}\right)\right\|_{0}\left\|d_{t} e_{\mathbf{u}}^{n}\right\|_{\alpha} \Delta t^{2} \\
& \leq \frac{v}{16}\left\|\bar{e}_{\mathbf{u}}^{n}\right\|_{\alpha+1}^{2} \Delta t+\frac{1}{16}\left\|d_{t} e_{\mathbf{u}}^{n}\right\|_{\alpha}^{2} \Delta t^{2}+c\left\|A_{h} \mathbf{u}_{h}\left(t_{n-1}\right)\right\|_{0}^{2}\left(\left\|e_{\mathbf{u}}^{n-1}\right\|_{\alpha}^{2}+\frac{v}{2}\left\|e_{\mathbf{u}}^{n-1}\right\|_{\alpha+1}^{2} \Delta t\right) \Delta t, \\
& 2\left|b\left(\bar{e}_{\mathbf{u}}^{n-1}, \bar{e}_{\mathbf{u}}^{n-1}, A_{h}^{\alpha} e_{\mathbf{u}}^{n}\right)\right| \Delta t \\
& \leq \frac{v}{16}\left\|e_{\mathbf{u}}^{n}\right\|_{\alpha+1}^{2} \Delta t+\frac{1}{16}\left\|d_{t} e_{\mathbf{u}}^{n}\right\|_{\alpha}^{2} \Delta t^{2}+c\left\|A_{h} \bar{e}_{\mathbf{u}}^{n-1}\right\|_{0}^{2}\left(\left\|e_{\mathbf{u}}^{n-1}+e_{\mathbf{u}}^{n-2}\right\|_{\alpha}^{2}+\left\|e_{\mathbf{u}}^{n-1}+e_{\mathbf{u}}^{n-2}\right\|_{\alpha+1}^{2} \Delta t\right) \Delta t, \\
& \left|b\left(d_{t} e_{\mathbf{u}}^{n-1}, \bar{e}_{\mathbf{u}}^{n-1}, A_{h}^{\alpha} e_{\mathbf{u}}^{n}\right)\right| \Delta t^{2}+\left|b\left(\bar{e}_{\mathbf{u}}^{n-1}, d_{t} e_{\mathbf{u}}^{n-1}, A_{h}^{\alpha} e_{\mathbf{u}}^{n}\right)\right| \Delta t^{2} \\
& \leq \frac{v}{16}\left\|\bar{e}_{\mathbf{u}}^{n}\right\|_{\alpha+1}^{2} \Delta t+\frac{1}{16}\left\|d_{t} e_{\mathbf{u}}^{n}\right\|_{\alpha}^{2} \Delta t^{2}+c\left\|A_{h} \bar{e}_{\mathbf{u}}^{n-1}\right\|_{0}^{2}\left(\left\|e_{\mathbf{u}}^{n-1}-e_{\mathbf{u}}^{n-2}\right\|_{\alpha}^{2}+\left\|e_{\mathbf{u}}^{n-1}-e_{\mathbf{u}}^{n-2}\right\|_{\alpha+1}^{2} \Delta t\right) \Delta t, \\
& \frac{1}{2}\left|b\left(d_{t} e_{\mathbf{u}}^{n-1}, d_{t} e_{\mathbf{u}}^{n-1}, A_{h}^{\alpha} \bar{e}_{\mathbf{u}}^{n}\right)\right| \Delta t^{3} \\
& \leq \frac{v}{16}\left\|\bar{e}_{\mathbf{u}}^{n}\right\|_{\alpha+1}^{2} \Delta t+\frac{1}{16}\left\|d_{t} e_{\mathbf{u}}^{n-1}\right\|_{\alpha}^{2} \Delta t^{2}+c\left\|e_{\mathbf{u}}^{n-1}-e_{\mathbf{u}}^{n-2}\right\|_{1}^{4}\left\|e_{\mathbf{u}}^{n-1}-e_{\mathbf{u}}^{n-2}\right\|_{\alpha+1}^{2} \Delta t^{2}, \quad \alpha=0,1, \\
& \frac{1}{2}\left|b\left(d_{t} e_{\mathbf{u}}^{n-1}, d_{t} e_{\mathbf{u}}^{n-1}, A_{h}^{-1} \bar{e}_{\mathbf{u}}^{n}\right)\right| \Delta t^{3} \\
& \leq \frac{v}{16}\left\|\bar{e}_{\mathbf{u}}^{n}\right\|_{0}^{2} \Delta t+c\left\|e_{\mathbf{u}}^{n-1}-e_{\mathbf{u}}^{n-2}\right\|_{1}^{2}\left\|e_{\mathbf{u}}^{n-1}-e_{\mathbf{u}}^{n-2}\right\|_{0}^{2} \Delta t^{2}, \\
& \frac{1}{4}\left|b\left(d_{t} e_{\mathbf{u}}^{n-1}, d_{t} e_{\mathbf{u}}^{n-1}, A_{h}^{\alpha} d_{t} e_{\mathbf{u}}^{n}\right)\right| \Delta t^{4} \\
& \leq \frac{1}{16}\left\|d_{t} e_{\mathbf{u}}^{n}\right\|_{\alpha}^{2} \Delta t^{2}+\frac{1}{16}\left\|d_{t} e_{\mathbf{u}}^{n-1}\right\|_{\alpha}^{2} \Delta t^{2}+c\left\|A_{h}\left(e_{\mathbf{u}}^{n-1}-e_{\mathbf{u}}^{n-2}\right)\right\|_{0}^{4}\left\|e_{\mathbf{u}}^{n-1}-e_{\mathbf{u}}^{n-2}\right\|_{\alpha+1}^{2} \Delta t^{4}, \quad \alpha=0,1, \\
& \frac{1}{4}\left|b\left(d_{t} e_{\mathbf{u}}^{n-1}, d_{t} e_{\mathbf{u}}^{n-1}, A_{h}^{-1} d_{t} e_{\mathbf{u}}^{n}\right)\right| \Delta t^{4} \\
& \leq \frac{1}{16}\left\|d_{t} e_{\mathbf{u}}^{n}\right\|_{-1}^{2} \Delta t^{2}+\frac{1}{16}\left\|d_{t} e_{\mathbf{u}}^{n-1}\right\|_{0}^{2} \Delta t^{3}+c\left\|A_{h}\left(e_{\mathbf{u}}^{n-1}-e_{\mathbf{u}}^{n-2}\right)\right\|_{0}^{2}\left\|e_{\mathbf{u}}^{n-1}-e_{\mathbf{u}}^{n-2}\right\|_{1}^{2}\left\|e_{\mathbf{u}}^{n-1}-e_{\mathbf{u}}^{n-2}\right\|_{0}^{2} \Delta t^{3} .
\end{aligned}
$$


For all $3 \leq n \leq N$ and $\alpha=0,1$, combining above inequalities with (4.4), we obtain

$$
\begin{aligned}
& \left(\left\|e_{\mathbf{u}}^{n}\right\|_{\alpha}^{2}+\frac{v}{2}\left\|e_{\mathbf{u}}^{n}\right\|_{\alpha+1}^{2} \Delta t\right)-\left(\left\|e_{\mathbf{u}}^{n-1}\right\|_{\alpha}^{2}+\frac{v}{2}\left\|e_{\mathbf{u}}^{n-1}\right\|_{\alpha+1}^{2} \Delta t\right)+\frac{5}{8}\left\|d_{t} e_{\mathbf{u}}^{n}\right\|_{\alpha}^{2} \Delta t^{2} \\
& \quad-\frac{1}{8}\left\|d_{t} e_{\mathbf{u}}^{n-1}\right\|_{\alpha}^{2} \Delta t^{2}+v\left\|\bar{e}_{\mathbf{u}}^{n}\right\|_{\alpha+1}^{2} \Delta t \\
& \leq \frac{1}{2} \widehat{b}_{n-1}\left(\left\|e_{\mathbf{u}}^{n-1}\right\|_{\alpha}^{2}+\frac{v}{2}\left\|e_{\mathbf{u}}^{n-1}\right\|_{\alpha+1}^{2} \Delta t\right) \Delta t+\frac{1}{2} \widehat{c}_{n-2}\left(\left\|e_{\mathbf{u}}^{n-2}\right\|_{\alpha}^{2}+\frac{v}{2}\left\|e_{\mathbf{u}}^{n-2}\right\|_{\alpha+1}^{2} \Delta t\right) \Delta t \\
& \quad+8 v^{-1}\left\|A_{h}^{\frac{\alpha-1}{2}} P_{h} e_{n}\right\|_{0}^{2} \Delta t+16\left\|A_{h}^{\frac{\alpha}{2}} P_{h} e_{n}\right\|_{0}^{2} \Delta t^{2} .
\end{aligned}
$$

As $\alpha=-1$, we have

$$
\begin{aligned}
& \left(\left\|e_{\mathbf{u}}^{n}\right\|_{-1}^{2}+\frac{v}{2}\left\|e_{\mathbf{u}}^{n}\right\|_{0}^{2} \Delta t\right)-\left(\left\|e_{\mathbf{u}}^{n-1}\right\|_{-1}^{2}+\frac{v}{2}\left\|e_{\mathbf{u}}^{n-1}\right\|_{0}^{2} \Delta t\right) \\
& \quad+\frac{5}{8}\left\|d_{t} e_{\mathbf{u}}^{n}\right\|_{-1}^{2} \Delta t^{2}-\frac{1}{8}\left\|d_{t} e_{\mathbf{u}}^{n-1}\right\|_{-1}^{2} \Delta t^{2}+v\left\|\bar{e}_{\mathbf{u}}^{n}\right\|_{0}^{2} \Delta t \\
& \leq \frac{1}{2} \widehat{b}_{n-1}\left(\left\|e_{\mathbf{u}}^{n-1}\right\|_{-1}^{2}+\frac{v}{2}\left\|e_{\mathbf{u}}^{n-1}\right\|_{0}^{2} \Delta t\right) \Delta t+\frac{1}{2} \widehat{c}_{n-2}\left(\left\|e_{\mathbf{u}}^{n-2}\right\|_{-1}^{2}+\frac{v}{2}\left\|e_{\mathbf{u}}^{n-2}\right\|_{0}^{2} \Delta t\right) \Delta t \\
& \quad+16\left\|A_{h}^{\frac{\alpha}{2}} P_{h} e_{n}\right\|_{0}^{2} \Delta t^{2}+c\left\|d_{t} e_{\mathbf{u}}^{n-1}\right\|_{0}^{2} \Delta t^{3} \\
& \quad+c\left\|e_{\mathbf{u}}^{n-1}-e_{\mathbf{u}}^{n-2}\right\|_{1}^{2}\left\|e_{\mathbf{u}}^{n-1}-e_{\mathbf{u}}^{n-2}\right\|_{0}^{2} \Delta t+8 v^{-1}\left\|A_{h}^{\frac{\alpha-1}{2}} P_{h} e_{n}\right\|_{0}^{2} \Delta t,
\end{aligned}
$$

where

$$
\begin{aligned}
\widehat{b}_{n-1}=c \| & A_{h} \mathbf{u}_{h}\left(t_{n-1}\right)\left\|_{0}^{2}+c\right\| A_{h} \bar{e}_{\mathbf{u}}^{n-1}\left\|_{0}^{2}+c\right\| e_{\mathbf{u}}^{n-1}-e_{\mathbf{u}}^{n-2}\left\|_{1}^{4}+c\right\| A_{h}\left(e_{\mathbf{u}}^{n-1}-e_{\mathbf{u}}^{n-2}\right) \|_{0}^{4} \Delta t^{2}, \\
\widehat{c}_{n-2}= & c\left\|A_{h} \bar{e}_{\mathbf{u}}^{n-1}\right\|_{0}^{2}+c\left\|e_{\mathbf{u}}^{n-1}-e_{\mathbf{u}}^{n-2}\right\|_{1}^{4}+c H(\alpha)\left\|A_{h}\left(e_{\mathbf{u}}^{n-1}-e_{\mathbf{u}}^{n-2}\right)\right\|_{0}^{4} \Delta t^{2} \\
& +c H(-\alpha)\left\|A_{h}\left(e_{\mathbf{u}}^{n-1}-e_{\mathbf{u}}^{n-2}\right)\right\|_{0}^{2}\left\|e_{\mathbf{u}}^{n-1}-e_{\mathbf{u}}^{n-2}\right\|_{1}^{2} \Delta t .
\end{aligned}
$$

Here and below, $H(\alpha)=1$ for all $\alpha>0$ and $H(\alpha)=0$ for all $\alpha<0$.

Noting the fact that $\widehat{d}_{n}=\frac{1}{2}\left(\widehat{b}_{n}+\widehat{c}_{n}\right)$, by Theorems 2.2 and 3.2, we have

$$
\Delta t \sum_{n=1}^{N} \widehat{d}_{n} \leq C .
$$

Thanks to Lemma 4.1 and summing (4.5) from $n=3$ to $m$, we get

$$
\begin{gathered}
\left\|e_{\mathbf{u}}^{m}\right\|_{\alpha}^{2}+\frac{v}{2}\left\|e_{\mathbf{u}}^{m}\right\|_{\alpha+1}^{2} \Delta t+\Delta t \sum_{n=3}^{m}\left(\frac{1}{2}\left\|d_{t} e_{\mathbf{u}}^{n}\right\|_{\alpha}^{2} \Delta t+v\left\|\bar{e}_{\mathbf{u}}^{n}\right\|_{\alpha+1}^{2}\right) \\
\leq\left\|e_{\mathbf{u}}^{2}\right\|_{\alpha}^{2}+\frac{v}{2}\left\|e_{\mathbf{u}}^{2}\right\|_{\alpha+1}^{2} \Delta t+\frac{1}{8}\left\|d_{t} e_{\mathbf{u}}^{2}\right\|_{\alpha}^{2} \Delta t^{2}+\Delta t \sum_{n=1}^{m-1} \widehat{d}_{n}\left(\left\|e_{\mathbf{u}}^{n}\right\|_{\alpha}^{2}+\frac{v}{2}\left\|e_{\mathbf{u}}^{n}\right\|_{\alpha+1}^{2} \Delta t\right) \\
\quad+v^{-1} \Delta t \sum_{n=3}^{m}\left(8\left\|A_{h}^{\frac{\alpha-1}{2}} P_{h} e_{n}\right\|_{0}^{2}+16 v\left\|A_{h}^{\frac{\alpha}{2}} P_{h} e_{n}\right\|_{0}^{2} \Delta t\right) \\
\leq \Delta t \sum_{n=1}^{m-1} \widehat{d}_{n}\left(\left\|e_{\mathbf{u}}^{n}\right\|_{\alpha}^{2}+\frac{v}{2}\left\|e_{\mathbf{u}}^{n}\right\|_{\alpha+1}^{2} \Delta t\right)+C \Delta t^{1-\alpha} .
\end{gathered}
$$


For all $3 \leq m \leq N$ with $\alpha=0,1$. Using (4.7) and Lemma 2.2, we deduce that

$$
\left\|e_{\mathbf{u}}^{m}\right\|_{\alpha}^{2}+\frac{v}{2}\left\|e_{\mathbf{u}}^{m}\right\|_{\alpha+1}^{2} \Delta t+\Delta t \sum_{n=3}^{m}\left(\frac{1}{2}\left\|d_{t} e_{\mathbf{u}}^{n}\right\|_{\alpha}^{2} \Delta t+v\left\|\bar{e}_{\mathbf{u}}^{n}\right\|_{\alpha+1}^{2}\right) \leq C \Delta t^{1-\alpha},
$$

with $\alpha=-1,0,1$, choosing $\psi_{h}=2 A_{h}^{\alpha} e_{T}^{n} \Delta t \in W_{h}, \mathbf{v}_{h}=0, q_{h}=0$ in (4.2), it yields

$$
\begin{aligned}
& \left(d_{t} e_{T}^{n}, 2 A_{h}^{\alpha} e_{T}^{n} \Delta t\right)+\tilde{a}\left(\bar{e}_{T}^{n}, 2 A_{h}^{\alpha} e_{T}^{n} \Delta t\right)+2 \tilde{b}\left(e_{\mathbf{u}}^{n-1}, T_{h}\left(t_{n-1}\right), A_{h}^{\alpha} e_{T}^{n}\right) \Delta t \\
\quad & +2 \tilde{b}\left(\mathbf{u}_{h}\left(t_{n-1}\right), e_{T}^{n-1}, A_{h}^{\alpha} e_{T}^{n}\right) \Delta t-2 \tilde{b}\left(\bar{e}_{\mathbf{u}}^{n-1}, \bar{e}_{T}^{n-1}, A_{h}^{\alpha} e_{T}^{n}\right) \Delta t-\tilde{b}\left(d_{t} e_{\mathbf{u}}^{n-1}, \bar{e}_{T}^{n-1}, A_{h}^{\alpha} e_{T}^{n}\right) \Delta t^{2} \\
& \quad-\tilde{b}\left(\bar{e}_{\mathbf{u}}^{n-1}, d_{t} e_{T}^{n-1}, A_{h}^{\alpha} e_{T}^{n}\right) \Delta t^{2}-\frac{1}{2} \tilde{b}\left(d_{t} e_{\mathbf{u}}^{n-1}, d_{t} e_{T}^{n-1}, A_{h}^{\alpha} e_{T}^{n}\right) \Delta t^{3} \\
= & \left(e_{n}^{\prime}, 2 A_{h}^{\alpha} e_{T}^{n} \Delta t\right) .
\end{aligned}
$$

By Lemma 2.2, the Cauchy inequality and (2.2b), we obtain

$$
\begin{aligned}
& 2\left|\tilde{b}\left(e_{\mathbf{u}}^{n-1}, T_{h}\left(t_{n-1}\right), A_{h}^{\alpha} e_{T}^{n}\right)\right| \Delta t+2\left|\tilde{b}\left(\mathbf{u}_{h}\left(t_{n-1}\right), e_{T}^{n-1}, A_{h}^{\alpha} e_{T}^{n}\right)\right| \Delta t \\
& =2\left|\tilde{b}\left(e_{\mathbf{u}}^{n-1}, T_{h}\left(t_{n-1}\right), A_{h}^{\alpha} \bar{e}_{T}^{n}+\frac{1}{2} A_{h}^{\alpha} d_{t} e_{T}^{n} \Delta t\right)\right| \Delta t+2\left|\tilde{b}\left(\mathbf{u}_{h}\left(t_{n-1}\right), e_{T}^{n-1}, A_{h}^{\alpha} \bar{e}_{T}^{n}+\frac{1}{2} A_{h}^{\alpha} d_{t} e_{T}^{n} \Delta t\right)\right| \Delta t \\
& \leq 2 \widetilde{C} \gamma_{0}\left\|e_{\mathbf{u}}^{n-1}\right\|_{\alpha}\left\|A_{h} T_{h}\left(t_{n-1}\right)\right\|_{0}\left\|\bar{e}_{T}^{n}\right\|_{\alpha+1} \Delta t+\widetilde{C} \gamma_{0}\left\|e_{\mathbf{u}}^{n-1}\right\|_{\alpha+1}\left\|A_{h} T_{h}\left(t_{n-1}\right)\right\|_{0}\left\|d_{t} e_{T}^{n}\right\|_{\alpha} \Delta t^{2} \\
& +2 \widetilde{C} \gamma_{0}\left\|e_{T}^{n-1}\right\|_{\alpha}\left\|A_{h} \mathbf{u}_{h}\left(t_{n-1}\right)\right\|_{0}\left\|\bar{e}_{T}^{n}\right\|_{\alpha+1} \Delta t+\widetilde{C} \gamma_{0}\left\|e_{T}^{n-1}\right\|_{\alpha+1}\left\|A_{h} \mathbf{u}_{h}\left(t_{n-1}\right)\right\|_{0}\left\|d_{t} e_{T}^{n}\right\|_{\alpha} \Delta t^{2} \\
& \leq \operatorname{Pr}^{-1} v / 16\left\|\bar{e}_{T}^{n}\right\|_{\alpha+1}^{2} \Delta t+1 / 16\left\|d_{t} e_{T}^{n}\right\|_{\alpha}^{2} \Delta t^{2}+c\left\|A_{h} \mathbf{u}_{h}\left(t_{n-1}\right)\right\|_{0}^{2}\left(\left\|e_{T}^{n-1}\right\|_{\alpha}^{2}\right. \\
& \left.+\frac{P r^{-1} v}{2}\left\|e_{T}^{n-1}\right\|_{\alpha+1}^{2} \Delta t\right) \Delta t+c\left\|A_{h} T_{h}\left(t_{n-1}\right)\right\|_{0}^{2}\left(\left\|e_{\mathbf{u}}^{n-1}\right\|_{\alpha}^{2}+\frac{\operatorname{Pr}^{-1} v}{2}\left\|e_{\mathbf{u}}^{n-1}\right\|_{\alpha+1}^{2} \Delta t\right) \Delta t, \\
& \left|\tilde{b}\left(d_{t} e_{\mathbf{u}}^{n-1}, \bar{e}_{T}^{n-1}, A_{h}^{\alpha} e_{T}^{n}\right)\right| \Delta t^{2}+\left|\tilde{b}\left(\bar{e}_{\mathbf{u}}^{n-1}, d_{t} e_{T}^{n-1}, A_{h}^{\alpha} e_{T}^{n}\right)\right| \Delta t^{2} \\
& \leq P r^{-1} v / 16\left\|\bar{e}_{T}^{n}\right\|_{\alpha+1}^{2} \Delta t+c\left\|A_{h} \bar{e}_{T}^{n-1}\right\|_{0}^{2}\left(\left\|e_{\mathbf{u}}^{n-1}-e_{\mathbf{u}}^{n-2}\right\|_{\alpha}^{2}+\left\|e_{\mathbf{u}}^{n-1}-e_{\mathbf{u}}^{n-2}\right\|_{\alpha+1}^{2} \Delta t\right) \Delta t \\
& +1 / 16\left\|d_{t} e_{T}^{n}\right\|_{\alpha}^{2} \Delta t^{2}+c\left\|A_{h} \bar{e}_{\mathbf{u}}^{n-1}\right\|_{0}^{2}\left(\left\|e_{T}^{n-1}-e_{T}^{n-2}\right\|_{\alpha}^{2}+\left\|e_{T}^{n-1}-e_{T}^{n-2}\right\|_{\alpha+1}^{2} \Delta t\right) \Delta t, \\
& 2\left|\tilde{b}\left(\bar{e}_{\mathbf{u}}^{n-1}, \bar{e}_{T}^{n-1}, A_{h}^{\alpha} e_{T}^{n}\right)\right| \Delta t \\
& \leq \operatorname{Pr}^{-1} \nu / 16\left\|\bar{e}_{T}^{n}\right\|_{\alpha+1}^{2} \Delta t+1 / 16\left\|d_{t} e_{T}^{n}\right\|_{\alpha}^{2} \Delta t^{2} \\
& +c\left\|A_{h} \bar{e}_{\mathbf{u}}^{n-1}\right\|_{0}^{2}\left(\left\|e_{T}^{n-1}+e_{T}^{n-2}\right\|_{\alpha}^{2}+\left\|e_{T}^{n-1}+e_{T}^{n-2}\right\|_{\alpha+1}^{2} \Delta t\right) \Delta t, \\
& \frac{1}{2}\left|\tilde{b}\left(d_{t} e_{\mathbf{u}}^{n-1}, d_{t} e_{T}^{n-1}, A_{h}^{\alpha} \bar{e}_{T}^{n}\right)\right| \Delta t^{3} \\
& \leq P r^{-1} v / 16\left\|\bar{e}_{T}^{n}\right\|_{\alpha+1}^{2} \Delta t+1 / 16\left\|d_{t} e_{T}^{n-1}\right\|_{\alpha}^{2} \Delta t^{2}+c \| e_{\mathbf{u}}^{n-1} \\
& -e_{\mathbf{u}}^{n-2}\left\|_{1}^{4}\right\| e_{T}^{n-1}-e_{T}^{n-2} \|_{\alpha+1}^{2} \Delta t^{2}, \quad \alpha=0,1, \\
& \frac{1}{4}\left|\tilde{b}\left(d_{t} e_{\mathbf{u}}^{n-1}, d_{t} e_{T}^{n-1}, A_{h}^{\alpha} d_{t} e_{T}^{n}\right)\right| \Delta t^{4} \\
& \leq 1 / 16\left\|d_{t} e_{T}^{n}\right\|_{\alpha}^{2} \Delta t^{2}+1 / 16\left\|d_{t} e_{T}^{n-1}\right\|_{\alpha}^{2} \Delta t^{2}+c \| A_{h}\left(e_{\mathbf{u}}^{n-1}\right. \\
& \left.-e_{\mathbf{u}}^{n-2}\right)\left\|_{0}^{4}\right\| e_{T}^{n-1}-e_{T}^{n-2} \|_{\alpha+1}^{2} \Delta t^{4}, \quad \alpha=0,1, \\
& \frac{1}{2}\left|\tilde{b}\left(d_{t} e_{\mathbf{u}}^{n-1}, d_{t} e_{T}^{n-1}, A_{h}^{-1} \bar{e}_{T}^{n}\right)\right| \Delta t^{3}
\end{aligned}
$$




$$
\begin{aligned}
\leq & \operatorname{Pr}^{-1} v / 16\left\|\bar{e}_{T}^{n}\right\|_{0}^{2} \Delta t+c\left\|e_{\mathbf{u}}^{n-1}-e_{\mathbf{u}}^{n-2}\right\|_{1}^{2}\left\|e_{T}^{n-1}-e_{T}^{n-2}\right\|_{0}^{2} \Delta t^{2}, \\
& \frac{1}{4}\left|\tilde{b}\left(d_{t} e_{\mathbf{u}}^{n-1}, d_{t} e_{T}^{n-1}, A_{h}^{-1} d_{t} e_{T}^{n}\right)\right| \Delta t^{4} \\
\leq & 1 / 16\left\|d_{t} e_{T}^{n}\right\|_{-1}^{2} \Delta t^{2}+1 / 16\left\|d_{t} e_{T}^{n-1}\right\|_{0}^{2} \Delta t^{3} \\
& \quad+c\left\|A_{h}\left(e_{\mathbf{u}}^{n-1}-e_{\mathbf{u}}^{n-2}\right)\right\|_{0}^{2}\left\|e_{T}^{n-1}-e_{T}^{n-2}\right\|_{1}^{2}\left\|e_{\mathbf{u}}^{n-1}-e_{\mathbf{u}}^{n-2}\right\|_{0}^{2} \Delta t^{3}, \\
& 2\left|\left(e_{n}^{\prime}, A_{h}^{\alpha} e_{T}^{n}\right)\right| \Delta t \\
\leq & \frac{\operatorname{Pr}^{-1} v}{8}\left\|\bar{e}_{T}^{n}\right\|_{\alpha+1}^{2} \Delta t+\frac{1}{16}\left\|d_{t} e_{T}^{n}\right\|_{\alpha}^{2} \Delta t^{2} \\
& \quad+8 \operatorname{Prv}^{-1}\left\|A_{h}^{\frac{\alpha-1}{2}} P_{h} e_{n}^{\prime}\right\|_{0}^{2} \Delta t+16\left\|A_{h}^{\frac{\alpha}{2}} e_{n}^{\prime}\right\|_{0}^{2} \Delta t^{2} .
\end{aligned}
$$

Then, for all $3 \leq n \leq N$ with $\alpha=0,1$, one finds

$$
\begin{aligned}
& \left(\left\|e_{T}^{n}\right\|_{\alpha}^{2}+\frac{P r^{-1} v}{2}\left\|e_{T}^{n}\right\|_{\alpha+1}^{2} \Delta t\right)-\left(\left\|e_{T}^{n-1}\right\|_{\alpha}^{2}+\frac{P^{-1} v}{2}\left\|e_{T}^{n-1}\right\|_{\alpha+1}^{2} \Delta t\right) \\
& \quad+\frac{5}{8}\left\|d_{t} e_{T}^{n}\right\|_{\alpha}^{2} \Delta t^{2}-\frac{1}{8}\left\|d_{t} e_{T}^{n-1}\right\|_{\alpha}^{2} \Delta t^{2}+\operatorname{Pr}^{-1} v\left\|\bar{e}_{T}^{n}\right\|_{\alpha+1}^{2} \Delta t \\
& \leq \frac{1}{2} \widetilde{b}_{n-1}\left(\left\|e_{T}^{n-1}\right\|_{\alpha}^{2}+\frac{P^{-1} v}{2}\left\|e_{T}^{n-1}\right\|_{\alpha+1}^{2} \Delta t\right) \Delta t+\frac{1}{2} \widetilde{c}_{n-2}\left(\left\|e_{T}^{n-2}\right\|_{\alpha}^{2}+\frac{\operatorname{Pr}^{-1} v}{2}\left\|e_{T}^{n-2}\right\|_{\alpha+1}^{2} \Delta t\right) \Delta t \\
& \quad+c\left\|A_{h} T_{h}\left(t_{n-1}\right)\right\|_{0}^{2}\left(\left\|e_{\mathbf{u}}^{n-1}\right\|_{\alpha}^{2}+\frac{P r^{-1} v}{2}\left\|e_{\mathbf{u}}^{n-1}\right\|_{\alpha+1}^{2} \Delta t\right) \Delta t+8 \operatorname{Pr} v^{-1}\left\|A_{h}^{\frac{\alpha-1}{2}} P_{h} e_{n}^{\prime}\right\|_{0}^{2} \Delta t \\
& \quad+c\left\|A_{h} \bar{e}_{T}^{n-1}\right\|_{0}^{2}\left(\left\|e_{\mathbf{u}}^{n-1}-e_{\mathbf{u}}^{n-2}\right\|_{\alpha}^{2}+\left\|e_{\mathbf{u}}^{n-1}-e_{\mathbf{u}}^{n-2}\right\|_{\alpha+1}^{2} \Delta t\right) \Delta t+16\left\|A_{h}^{\frac{\alpha}{2}} P_{h} e_{n}^{\prime}\right\|_{0}^{2} \Delta t^{2},
\end{aligned}
$$

and with $\alpha=-1$, we have

$$
\begin{aligned}
& \left(\left\|e_{T}^{n}\right\|_{-1}^{2}+\frac{P r^{-1} v}{2}\left\|e_{T}^{n}\right\|_{0}^{2} \Delta t\right)-\left(\left\|e_{T}^{n-1}\right\|_{-1}^{2}+\frac{P^{-1} v}{2}\left\|e_{T}^{n-1}\right\|_{0}^{2} \Delta t\right) \\
& \quad+\frac{5}{8}\left\|d_{t} e_{T}^{n}\right\|_{-1}^{2} \Delta t^{2}-\frac{1}{8}\left\|d_{t} e_{T}^{n-1}\right\|_{-1}^{2} \Delta t^{2}+\operatorname{Pr}^{-1} v\left\|\bar{e}_{T}^{n}\right\|_{0}^{2} \Delta t \\
& \leq \frac{1}{2} \widetilde{b}_{n-1}\left(\left\|e_{T}^{n-1}\right\|_{-1}^{2}+\frac{\operatorname{Pr}^{-1} v}{2}\left\|e_{T}^{n-1}\right\|_{0}^{2} \Delta t\right) \Delta t+\frac{1}{2} \widetilde{c}_{n-2}\left(\left\|e_{T}^{n-2}\right\|_{-1}^{2}+\frac{\operatorname{Pr}^{-1} v}{2}\left\|e_{T}^{n-2}\right\|_{0}^{2} \Delta t\right) \Delta t \\
& \quad+c\left\|d_{t} e_{T}^{n-1}\right\|_{0}^{2} \Delta t^{3}+c\left\|e_{\mathbf{u}}^{n-1}-e_{\mathbf{u}}^{n-2}\right\|_{1}^{2}\left\|e_{T}^{n-1}-e_{T}^{n-2}\right\|_{0}^{2} \Delta t \\
& \quad+c\left\|A_{h} T_{h}\left(t_{n-1}\right)\right\|_{0}^{2}\left(\left\|e_{\mathbf{u}}^{n-1}\right\|_{-1}^{2}+\frac{\operatorname{Pr}^{-1} v}{2}\left\|e_{\mathbf{u}}^{n-1}\right\|_{0}^{2} \Delta t\right) \Delta t+8 \operatorname{Prv}^{-1}\left\|A_{h}^{\frac{\alpha-1}{2}} P_{h} e_{n}^{\prime}\right\|_{0}^{2} \Delta t \\
& \quad+c\left\|A_{h} \bar{e}_{T}^{n-1}\right\|_{0}^{2}\left(\left\|e_{\mathbf{u}}^{n-1}-e_{\mathbf{u}}^{n-2}\right\|_{-1}^{2}+\left\|e_{\mathbf{u}}^{n-1}-e_{\mathbf{u}}^{n-2}\right\|_{0}^{2} \Delta t\right) \Delta t+16\left\|A_{h}^{\frac{\alpha}{2}} P_{h} e_{n}^{\prime}\right\|_{0}^{2} \Delta t^{2}, \quad(4 .
\end{aligned}
$$

where

$$
\begin{aligned}
\widetilde{b}_{n-1}= & c\left\|A_{h} \mathbf{u}_{h}\left(t_{n-1}\right)\right\|_{0}^{2}+c\left\|A_{h} \bar{e}_{\mathbf{u}}^{n-1}\right\|_{0}^{2}+c\left\|e_{\mathbf{u}}^{n-1}-e_{\mathbf{u}}^{n-2}\right\|_{1}^{4}+c\left\|A_{h}\left(e_{\mathbf{u}}^{n-1}-e_{\mathbf{u}}^{n-2}\right)\right\|_{0}^{4} \Delta t^{2}, \\
\widetilde{c}_{n-2}= & c\left\|A_{h} \bar{e}_{\mathbf{u}}^{n-1}\right\|_{0}^{2}+c\left\|e_{\mathbf{u}}^{n-1}-e_{\mathbf{u}}^{n-2}\right\|_{1}^{4}+c H(\alpha)\left\|A_{h}\left(e_{\mathbf{u}}^{n-1}-e_{\mathbf{u}}^{n-2}\right)\right\|_{0}^{4} \Delta t^{2} \\
& +c H(-\alpha)\left\|A_{h}\left(e_{\mathbf{u}}^{n-1}-e_{\mathbf{u}}^{n-2}\right)\right\|_{0}^{2}\left\|e_{T}^{n-1}-e_{T}^{n-2}\right\|_{1}^{2} \Delta t .
\end{aligned}
$$


Noting $\widetilde{d}_{n}=\frac{1}{2}\left(\widetilde{b}_{n}+\widetilde{c}_{n}\right)$, using Theorems 2.2 and 3.2, we have

$$
\Delta t \sum_{n=1}^{N} \widetilde{d}_{n} \leq C
$$

Applying Lemma 4.1 and summing (4.9) from $n=3$ to $m$, we get

$$
\begin{aligned}
& \left\|e_{T}^{m}\right\|_{\alpha}^{2}+\frac{P^{-1} v}{2}\left\|e_{T}^{m}\right\|_{\alpha+1}^{2} \Delta t+\Delta t \sum_{n=3}^{m}\left(\frac{1}{2}\left\|d_{t} e_{T}^{n}\right\|_{\alpha}^{2} \Delta t+\operatorname{Pr}^{-1} v\left\|\bar{e}_{T}^{n}\right\|_{\alpha+1}^{2}\right) \\
& \leq\left\|e_{T}^{2}\right\|_{\alpha}^{2}+\frac{P^{-1} v}{2}\left\|e_{T}^{2}\right\|_{\alpha+1}^{2} \Delta t+\frac{1}{8}\left\|d_{t} e_{T}^{2}\right\|_{\alpha}^{2} \Delta t^{2}+\Delta t \sum_{n=2}^{m-1} \widetilde{d}_{n}\left(\left\|e_{T}^{n}\right\|_{\alpha}^{2}+\frac{\operatorname{Pr}^{-1} v}{2}\left\|e_{T}^{n}\right\|_{\alpha+1}^{2} \Delta t\right) \\
& \quad+c \sum_{n=3}^{m}\left\|A_{h} T_{h}\left(t_{n-1}\right)\right\|_{0}^{2}\left(\left\|e_{\mathbf{u}}^{n-1}\right\|_{\alpha}^{2}+\frac{\operatorname{Pr}^{-1} v}{2}\left\|e_{\mathbf{u}}^{n-1}\right\|_{\alpha+1}^{2} \Delta t\right) \Delta t \\
& +c \sum_{n=3}^{m}\left\|A_{h} \bar{e}_{T}^{n-1}\right\|_{0}^{2}\left(\left\|e_{\mathbf{u}}^{n-1}-e_{\mathbf{u}}^{n-2}\right\|_{\alpha}^{2}+\left\|e_{\mathbf{u}}^{n-1}-e_{\mathbf{u}}^{n-2}\right\|_{\alpha+1}^{2} \Delta t\right) \Delta t \\
& \quad+\operatorname{Prv}^{-1} \Delta t \sum_{n=3}^{m}\left(8\left\|A_{h}^{\frac{\alpha-1}{2}} P_{h} e_{n}^{\prime}\right\|_{0}^{2}+16 \operatorname{Pr}^{-1} v\left\|A_{h}^{\frac{\alpha}{2}} P_{h} e_{n}^{\prime}\right\|_{0}^{2} \Delta t\right) \\
& \leq \Delta t \sum_{n=2}^{m-1} \widetilde{d}_{n}\left(\left\|e_{T}^{n}\right\|_{\alpha}^{2}+\frac{\operatorname{Pr}^{-1} v}{2}\left\|e_{T}^{n}\right\|_{\alpha+1}^{2} \Delta t\right)+C \Delta t^{1-\alpha} .
\end{aligned}
$$

For all $3 \leq m \leq N$ with $\alpha=0,1$. By Lemma 2.2 and (4.11) to above inequality, we get

$$
\left\|e_{T}^{m}\right\|_{\alpha}^{2}+\frac{\operatorname{Pr}^{-1} v}{2}\left\|e_{T}^{m}\right\|_{\alpha+1}^{2} \Delta t+\Delta t \sum_{n=3}^{m}\left(\frac{1}{2}\left\|d_{t} e_{T}^{n}\right\|_{\alpha}^{2} \Delta t+\operatorname{Pr}^{-1} v\left\|\bar{e}_{T}^{n}\right\|_{\alpha+1}^{2}\right) \leq C \Delta t^{1-\alpha}
$$

Substituting (4.8) into (4.12) obtains (4.3a) with $\alpha=0,1$. From (4.3a) with $\alpha=0$, one finds

$$
\Delta t^{3} \sum_{n=2}^{m}\left\|d_{t} e_{\mathbf{u}}^{n}, d_{t} e_{T}^{n}\right\|_{0}^{2} \leq C \Delta t^{2}
$$

Finally, we deduce (4.3a) for $\alpha=-1$ by using (4.6) and (4.10).

Theorem 4.2. Under the assumptions of (A1)-(A3) and (3.9), for all $1 \leq m \leq N$, we have

$$
\begin{aligned}
& \left\|e_{u}^{m}, e_{T}^{m}\right\|_{-2}^{2}+\min \left\{v, \operatorname{Pr}^{-1} v\right\}\left\|e_{u}^{m}, e_{T}^{m}\right\|_{-1}^{2} \Delta t \\
& \quad+\Delta t \sum_{n=1}^{m}\left(\frac{1}{2}\left\|d_{t} e_{u}^{n}, d_{t} e_{T}^{n}\right\|_{-2}^{2} \Delta t+\min \left\{v, \operatorname{Pr}^{-1} v\right\}\left\|\bar{e}_{u}^{n}, \bar{e}_{T}^{n}\right\|_{-1}^{2}\right) \leq C \Delta t^{3} .
\end{aligned}
$$


Proof. Firstly, taking $\mathbf{v}_{h}=2 A_{h}^{-2} e_{\mathbf{u}}^{n} \Delta t \in V_{h}, q_{h}=0, \psi_{h}=0$ in (4.2), we obtain

$$
\begin{aligned}
& \left\|e_{\mathbf{u}}^{n}\right\|_{-2}^{2}-\left\|e_{\mathbf{u}}^{n-1}\right\|_{-2}^{2}+\left\|d_{t} e_{\mathbf{u}}^{n}\right\|_{-2}^{2} \Delta t^{2}+\frac{v}{2}\left(\left\|e_{\mathbf{u}}^{n}\right\|_{-1}^{2}-\left\|e_{\mathbf{u}}^{n-1}\right\|_{-1}^{2}\right) \Delta t \\
& \quad+2 v\left\|\bar{e}_{\mathbf{u}}^{n}\right\|_{-1}^{2} \Delta t+2 b\left(e_{\mathbf{u}}^{n-1}, \mathbf{u}_{h}\left(t_{n-1}\right), A_{h}^{-2} e_{\mathbf{u}}^{n}\right) \Delta t+2 b\left(\mathbf{u}_{h}\left(t_{n-1}\right), e_{\mathbf{u}}^{n-1}, A_{h}^{-2} e_{\mathbf{u}}^{n}\right) \Delta t \\
& \quad-2 b\left(\bar{e}_{\mathbf{u}}^{n-1}, \bar{e}_{\mathbf{u}}^{n-1}, A_{h}^{-2} e_{\mathbf{u}}^{n}\right) \Delta t-b\left(\bar{e}_{\mathbf{u}}^{n-1}, d_{t} e_{\mathbf{u}}^{n-1}, A_{h}^{-2} e_{\mathbf{u}}^{n}\right) \Delta t^{2} \\
& \quad-b\left(d_{t} e_{\mathbf{u}}^{n-1}, \bar{e}_{\mathbf{u}}^{n-1}, A_{h}^{-2} e_{\mathbf{u}}^{n}\right) \Delta t^{2}-\frac{1}{2} b\left(d_{t} e_{\mathbf{u}}^{n-1}, d_{t} e_{\mathbf{u}}^{n-1}, A_{h}^{-2} e_{\mathbf{u}}^{n}\right) \Delta t^{3} \\
& =2\left(e_{n}, A_{h}^{-2} e_{\mathbf{u}}^{n}\right) \Delta t .
\end{aligned}
$$

Noting the fact that

$$
e_{\phi}^{n}=d_{t} e_{\phi}^{n} \Delta t+e_{\phi}^{n-1}, \quad e_{\phi}^{n}=\bar{e}_{\phi}^{n}+\frac{1}{2} d_{t} e_{\phi}^{n} \Delta t, \quad e_{\phi}^{n-1}=\bar{e}_{\phi}^{n}-\frac{1}{2} d_{t} e_{\phi}^{n} \Delta t, \quad \phi \text { takes } \mathbf{u} \text { or } T,
$$

and using Lemma 2.2, the Cauchy inequality and (2.2b), we have

$$
\begin{aligned}
& 2\left|b\left(e_{\mathbf{u}}^{n-1}, \mathbf{u}_{h}\left(t_{n-1}\right), A_{h}^{-2} d_{t} e_{\mathbf{u}}^{n}\right)\right| \Delta t^{2}+2\left|b\left(\mathbf{u}_{h}\left(t_{n-1}\right), e_{\mathbf{u}}^{n-1}, A_{h}^{-2} d_{t} e_{\mathbf{u}}^{n}\right)\right| \Delta t^{2} \\
& \leq 2 \widehat{C} \gamma_{0}\left\|e_{\mathbf{u}}^{n-1}\right\|_{-1}\left\|A_{h} \mathbf{u}_{h}\left(t_{n-1}\right)\right\|_{0}\left\|d_{t} e_{\mathbf{u}}^{n}\right\|_{-2} \Delta t^{2} \\
& \leq \frac{1}{16}\left\|d_{t} e_{\mathbf{u}}^{n}\right\|_{-2}^{2} \Delta t^{2}+c\left\|A_{h} \mathbf{u}_{h}\left(t_{n-1}\right)\right\|_{0}^{2}\left\|e_{\mathbf{u}}^{n-1}\right\|_{-1}^{2} \Delta t^{2}, \\
& 2\left|b\left(e_{\mathbf{u}}^{n-1}, \mathbf{u}_{h}\left(t_{n-1}\right), A_{h}^{-2} e_{\mathbf{u}}^{n-1}\right)\right| \Delta t+2\left|b\left(\mathbf{u}_{h}\left(t_{n-1}\right), e_{\mathbf{u}}^{n-1}, A_{h}^{-2} e_{\mathbf{u}}^{n-1}\right)\right| \Delta t \\
& \leq \frac{v}{8}\left\|{\overline{e_{\mathbf{u}}}}^{n}\right\|_{-1}^{2} \Delta t+\frac{1}{8}\left\|d_{t} e_{\mathbf{u}}^{n}\right\|_{-1}^{2} \Delta t^{3}+c\left\|A_{h} \mathbf{u}_{h}\left(t_{n-1}\right)\right\|_{0}^{2}\left\|e_{\mathbf{u}}^{n-1}\right\|_{-2}^{2} \Delta t, \\
& 2\left|b\left(\bar{e}_{\mathbf{u}}^{n-1}, \bar{e}_{\mathbf{u}}^{n-1}, A_{h}^{-2} d_{t} e_{\mathbf{u}}^{n}\right)\right| \Delta t^{2} \leq \frac{1}{16}\left\|d_{t} e_{\mathbf{u}}^{n}\right\|_{-2}^{2} \Delta t^{2}+c\left\|\bar{e}_{\mathbf{u}}^{n-1}\right\|_{0}^{2}\left\|\bar{e}_{\mathbf{u}}^{n-1}\right\|_{1}^{2} \Delta t^{2}, \\
& 2\left|b\left(\bar{e}_{\mathbf{u}}^{n-1}, \bar{e}_{\mathbf{u}}^{n-1}, A_{h}^{-2} e_{\mathbf{u}}^{n-1}\right)\right| \Delta t \leq \widehat{C} \gamma_{0}\left\|\bar{e}_{\mathbf{u}}^{n-1}\right\|_{-1}\left\|A_{h} \bar{e}_{\mathbf{u}}^{n-1}\right\|_{0}\left\|e_{\mathbf{u}}^{n-1}\right\|_{-2} \Delta t \\
& \leq \frac{v}{8}\left\|\bar{e}_{\mathbf{u}}^{n-1}\right\|_{-1}^{2} \Delta t+c\left\|A_{h} \bar{e}_{\mathbf{u}}^{n-1}\right\|_{0}^{2}\left\|e_{\mathbf{u}}^{n-1}\right\|_{-2}^{2} \Delta t, \\
& \left|b\left(d_{t} e_{\mathbf{u}}^{n-1}, \bar{e}_{\mathbf{u}}^{n-1}, A_{h}^{-2} d_{t} e_{\mathbf{u}}^{n}\right)\right| \Delta t^{3}+\left|b\left(\bar{e}_{\mathbf{u}}^{n-1}, d_{t} e_{\mathbf{u}}^{n-1}, A_{h}^{-2} d_{t} e_{\mathbf{u}}^{n}\right)\right| \Delta t^{3} \\
& \leq \frac{1}{16}\left\|d_{t} e_{\mathbf{u}}^{n}\right\|_{-2}^{2} \Delta t^{2}+c\left\|A_{h} \bar{e}_{\mathbf{u}}^{n-1}\right\|_{0}^{2}\left\|e_{\mathbf{u}}^{n-1}-e_{\mathbf{u}}^{n-2}\right\|_{-1}^{2} \Delta t^{2}, \\
& \left|b\left(d_{t} e_{\mathbf{u}}^{n-1}, \bar{e}_{\mathbf{u}}^{n-1}, A_{h}^{-2} e_{\mathbf{u}}^{n-1}\right)\right| \Delta t^{2}+\left|b\left(\bar{e}_{\mathbf{u}}^{n-1}, d_{t} e_{\mathbf{u}}^{n-1}, A_{h}^{-2} e_{\mathbf{u}}^{n-1}\right)\right| \Delta t^{2} \\
& \leq \frac{1}{8}\left\|d_{t} e_{\mathbf{u}}^{n-1}\right\|_{-1}^{2} \Delta t^{3}+c\left\|A_{h} \bar{e}_{\mathbf{u}}^{n-1}\right\|_{0}^{2}\left\|e_{\mathbf{u}}^{n-1}\right\|_{-2}^{2} \Delta t, \\
& \frac{1}{2}\left|b\left(d_{t} e_{\mathbf{u}}^{n-1}, d_{t} e_{\mathbf{u}}^{n-1}, A_{h}^{-2} d_{t} e_{\mathbf{u}}^{n}\right)\right| \Delta t^{4} \leq \frac{1}{4} \widehat{C} \gamma_{0}\left\|d_{t} e_{\mathbf{u}}^{n-1}\right\|_{0}\left\|d_{t} e_{\mathbf{u}}^{n-1}\right\|_{1}\left\|d_{t} e_{\mathbf{u}}^{n}\right\|_{-2} \Delta t^{4} \\
& \leq \frac{1}{16}\left\|d_{t} e_{\mathbf{u}}^{n}\right\|_{-2}^{2} \Delta t^{2}+c\left\|e_{\mathbf{u}}^{n-1}-e_{\mathbf{u}}^{n-2}\right\|_{1}^{2}\left\|d_{t} e_{\mathbf{u}}^{n-1}\right\|_{0}^{2} \Delta t^{4}, \\
& \frac{1}{2}\left|b\left(d_{t} e_{\mathbf{u}}^{n-1}, d_{t} e_{\mathbf{u}}^{n-1}, A_{h}^{-2} e_{\mathbf{u}}^{n-1}\right)\right| \Delta t^{3} \leq \frac{1}{4} \widehat{C} \gamma_{0}\left\|d_{t} e_{\mathbf{u}}^{n-1}\right\|_{0}\left\|d_{t} e_{\mathbf{u}}^{n-1}\right\|_{1}\left\|e_{\mathbf{u}}^{n-1}\right\|_{-2} \Delta t^{3}
\end{aligned}
$$




$$
\begin{aligned}
& \leq \frac{1}{8}\left\|d_{t} e_{\mathbf{u}}^{n-1}\right\|_{0}^{2} \Delta t^{4}+c\left\|d_{t} e_{\mathbf{u}}^{n-1}\right\|_{1}^{2}\left\|e_{\mathbf{u}}^{n-1}\right\|_{-2}^{2} \Delta t^{2}, \\
& \int_{t_{n-1}}^{t_{n}}\left(s-t_{n-1}\right)\left(t_{n}-s\right)\left(\mathbf{f}_{s s}, A_{h}^{-2}\left(d_{t} e_{\mathbf{u}}^{n} \Delta t+e_{\mathbf{u}}^{n-1}\right)\right) d s \\
& \leq \frac{1}{16}\left\|d_{t} e_{\mathbf{u}}^{n}\right\|_{-2}^{2} \Delta t^{2}+\left\|e_{\mathbf{u}}^{n-1}\right\|_{-2}^{2} \Delta t+c \Delta t^{3} \int_{t_{n-1}}^{t_{n}}\left(\left\|\mathbf{f}_{s s}\right\|_{0}^{2}+\Delta t\left\|\mathbf{f}_{s s}\right\|_{0}^{2}\right) d s, \\
& \kappa v^{2} \int_{t_{n-1}}^{t_{n}}\left(s-t_{n-1}\right)\left(t_{n}-s\right)\left(j T_{h s s}, A_{h}^{-2}\left(d_{t} e_{\mathbf{u}}^{n} \Delta t+e_{\mathbf{u}}^{n-1}\right)\right) d s \\
& \leq \frac{1}{16}\left\|d_{t} e_{\mathbf{u}}^{n}\right\|_{-2}^{2} \Delta t^{2}+\left\|e_{\mathbf{u}}^{n-1}\right\|_{-2}^{2} \Delta t+c \kappa^{2} v^{4} \Delta t^{3} \int_{t_{n-1}}^{t_{n}}\left(\left\|T_{h s s}\right\|_{0}^{2}+\Delta t\left\|T_{h s s}\right\|_{0}^{2}\right) d s, \\
& \kappa v^{2} \int_{t_{n-1}}^{t_{n}}\left(j T_{h}, A_{h}^{-2}\left(d_{t} e_{\mathbf{u}}^{n} \Delta t+e_{\mathbf{u}}^{n-1}\right)\right) d s \\
& \leq \frac{1}{16}\left\|d_{t} e_{\mathbf{u}}^{n}\right\|_{-2}^{2} \Delta t^{2}+\left\|e_{\mathbf{u}}^{n-1}\right\|_{-2}^{2} \Delta t+c \kappa^{2} v^{4} \Delta t^{3} \int_{t_{n-1}}^{t_{n}}\left(\left\|T_{h}\right\|_{0}^{2}+\Delta t\left\|T_{h}\right\|_{0}^{2}\right) d s, \\
& \Delta t^{2} \int_{t_{n-1}}^{t_{n}}\left|b_{s}\left(\mathbf{u}_{h}(s), \mathbf{u}_{h}(s), A_{h}^{-2}\left(d_{t} e_{\mathbf{u}}^{n} \Delta t+e_{\mathbf{u}}^{n-1}\right)\right)\right| d s \\
& \leq \widehat{C} \gamma_{0} \Delta t^{2} \int_{t_{n-1}}^{t_{n}}\left(\left\|\mathbf{u}_{h s}\right\|_{-1}\left\|A_{h} \mathbf{u}_{h}\right\|_{0}+\left\|\mathbf{u}_{h}\right\|_{0}\left\|\mathbf{u}_{h s}\right\|_{1}\right) d s\left(\left\|d_{t} e_{\mathbf{u}}^{n}\right\|_{-2}+\left\|e_{\mathbf{u}}^{n-1}\right\|_{-2}\right) \\
& \leq \frac{1}{16}\left\|d_{t} e_{\mathbf{u}}^{n}\right\|_{-2}^{2} \Delta t^{2}+c \int_{t_{n-1}}^{t_{n}}\left(\left\|A_{h} \mathbf{u}_{h}\right\|_{0}^{2}+\left\|\mathbf{u}_{h}\right\|_{0}^{2}\right) d s\left\|e_{\mathbf{u}}^{n-1}\right\|_{-2}^{2} \\
& +c \Delta t^{3} \int_{t_{n-1}}^{t_{n}}\left[\left(\Delta t+\Delta t^{2}\left\|A_{h} \mathbf{u}_{h}\right\|_{0}^{2}\right)\left\|\mathbf{u}_{h s}\right\|_{-1}^{2}+\left(\Delta t+\Delta t^{2}\left\|\mathbf{u}_{h}\right\|_{0}^{2}\right)\left\|\mathbf{u}_{h s}\right\|_{1}^{2}\right] d s, \\
& \int_{t_{n-1}}^{t_{n}}\left(s-t_{n-1}\right)\left(t_{n}-s\right) a\left(\mathbf{u}_{h s s}, A_{h}^{-2}\left(\frac{1}{2} d_{t} e_{\mathbf{u}}^{n} \Delta t+\bar{e}_{\mathbf{u}}^{n}\right)\right) d s \\
& \leq \frac{1}{16}\left\|d_{t} e_{\mathbf{u}}^{n}\right\|_{-2}^{2} \Delta t^{2}+\frac{v}{8}\left\|{\overline{e_{\mathbf{u}}}}^{n}\right\|_{-1}^{2} \Delta t+c \int_{t_{n-1}}^{t_{n}}\left(\Delta t^{2}\left\|\mathbf{u}_{h s s}\right\|_{0}^{2}+\Delta t\left\|\mathbf{u}_{h s s}\right\|_{-1}^{2}\right) d s, \\
& \Delta t^{2} \int_{t_{n-1}}^{t_{n}}\left|b_{s s}\left(\mathbf{u}_{h}, \mathbf{u}_{h}, A_{h}^{-2}\left(d_{t} e_{\mathbf{u}}^{n} \Delta t+e_{\mathbf{u}}^{n-1}\right)\right)\right| d s \\
& \leq \widehat{C} \gamma_{0} \Delta t^{2} \int_{t_{n-1}}^{t_{n}}\left(\left\|\mathbf{u}_{h s s}\right\|_{-1}\left\|A_{h} \mathbf{u}_{h}\right\|_{0}+\left\|\mathbf{u}_{h s}\right\|_{0}\left\|\mathbf{u}_{h s}\right\|_{1}\right) d s\left(\left\|d_{t} e_{\mathbf{u}}^{n}\right\|_{-2}+\left\|e_{\mathbf{u}}^{n-1}\right\|_{-2}\right) \\
& \leq \frac{1}{16}\left\|d_{t} e_{\mathbf{u}}^{n}\right\|_{-2}^{2} \Delta t^{2}+c \int_{t_{n-1}}^{t_{n}}\left(\left\|A_{h} \mathbf{u}_{h}\right\|_{0}^{2}+\left\|\mathbf{u}_{h s}\right\|_{0}^{2}\right) d s\left\|e_{\mathbf{u}}^{n-1}\right\|_{-2}^{2} \\
& +c \Delta t^{3} \int_{t_{n-1}}^{t_{n}}\left[\left(\Delta t+\Delta t^{2}\left\|A_{h} \mathbf{u}_{h}\right\|_{0}^{2}\right)\left\|\mathbf{u}_{h s s}\right\|_{-1}^{2}+\left(\Delta t+\Delta t^{2}\left\|\mathbf{u}_{h s}\right\|_{0}^{2}\right)\left\|\mathbf{u}_{h s}\right\|_{1}^{2}\right] d s .
\end{aligned}
$$

For all $3 \leq n \leq N$ and using the above inequalities with (4.13), we get

$$
\left(\left\|e_{\mathbf{u}}^{n}\right\|_{-2}^{2}+\frac{v}{2}\left\|e_{\mathbf{u}}^{n}\right\|_{-1}^{2} \Delta t\right)-\left(\left\|e_{\mathbf{u}}^{n-1}\right\|_{-2}^{2}+\frac{v}{2}\left\|e_{\mathbf{u}}^{n-1}\right\|_{-1}^{2} \Delta t\right)+\frac{1}{2}\left\|d_{t} e_{\mathbf{u}}^{n}\right\|_{-2}^{2} \Delta t^{2}+\frac{3 v}{2}\left\|e_{\mathbf{u}}^{n}\right\|_{-1}^{2} \Delta t
$$




$$
\begin{aligned}
\leq & \frac{1}{2} \widehat{b}_{n-1}^{\prime}\left(\left\|e_{\mathbf{u}}^{n-1}\right\|_{-2}^{2}+\frac{v}{2}\left\|e_{\mathbf{u}}^{n-1}\right\|_{-1}^{2} \Delta t\right) \Delta t^{2}+c\left\|A_{h} \bar{e}_{\mathbf{u}}^{n-1}\right\|_{0}^{2}\left\|e_{\mathbf{u}}^{n-1}-e_{\mathbf{u}}^{n-2}\right\|_{-1}^{2} \Delta t^{2}+c\left\|d_{t} e_{u}^{n}\right\|_{-1}^{2} \Delta t^{3} \\
& +c\left\|\bar{e}_{\mathbf{u}}^{n-1}\right\|_{0}^{2}\left\|\bar{e}_{\mathbf{u}}^{n-1}\right\|_{1}^{2} \Delta t+c\left(1+\left\|e_{\mathbf{u}}^{n-1}-e_{\mathbf{u}}^{n-2}\right\|_{1}^{2}\right)\left\|d_{t} e_{\mathbf{u}}^{n-1}\right\|_{0}^{2} \Delta t^{4}+c\left\|d_{t} e_{\mathbf{u}}^{n-1}\right\|_{-1}^{2} \Delta t^{3} \\
& +c \Delta t^{3} \int_{t_{n-1}}^{t_{n}}\left(\left\|\mathbf{f}_{s s}\right\|_{0}^{2}+\Delta t\left\|\mathbf{f}_{s s}\right\|_{0}^{2}\right) d s+c \kappa^{2} v^{4} \Delta t^{3} \int_{t_{n-1}}^{t_{n}}\left(\left\|T_{h s s}\right\|_{0}^{2}+\Delta t\left\|T_{h s s}\right\|_{0}^{2}\right) d s+\frac{v}{2}\left\|\bar{e}_{\mathbf{u}}^{n-1}\right\|_{-1}^{2} \Delta t \\
+ & c \Delta t^{2} \int_{t_{n-1}}^{t_{n}}\left(\Delta t^{2}\left\|\mathbf{u}_{h s s}\right\|_{0}^{2}+\Delta t\left\|\mathbf{u}_{h s s}\right\|_{-1}^{2}\right) d s+c \mathcal{K}^{2} v^{4} \Delta t^{3} \int_{t_{n-1}}^{t_{n}}\left(\left\|T_{h}\right\|_{0}^{2}+\Delta t\left\|T_{h}\right\|_{0}^{2}\right) d s \\
& +c \Delta t^{3} \int_{t_{n-1}}^{t_{n}}\left[\left(\Delta t+\Delta t^{2}\left\|A_{h} \mathbf{u}_{h}\right\|_{0}^{2}\right)\left\|\mathbf{u}_{h s s}\right\|_{-1}^{2}+\left(\Delta t+\Delta t^{2}\left\|\mathbf{u}_{h s}\right\|_{0}^{2}\right)\left\|\mathbf{u}_{h s}\right\|_{1}^{2}\right] d s, \\
& +c \Delta t^{3} \int_{t_{n-1}}^{t_{n}}\left[\left(\Delta t+\Delta t^{2}\left\|A_{h} \mathbf{u}_{h}\right\|_{0}^{2}\right)\left\|\mathbf{u}_{h s}\right\|_{-1}^{2}+\left(\Delta t+\Delta t^{2}\left\|\mathbf{u}_{h}\right\|_{0}^{2}\right)\left\|\mathbf{u}_{h s}\right\|_{1}^{2}\right] d s,
\end{aligned}
$$

where

$$
\widehat{b}_{n-1}^{\prime}=c\left(\left\|d_{t} e_{\mathbf{u}}^{n-1}\right\|_{1}^{2} \Delta t+\left\|A_{h} \mathbf{u}_{h}\left(t_{n-1}\right)\right\|_{0}^{2}+\left\|A_{h} \bar{e}_{\mathbf{u}}^{n-1}\right\|_{0}^{2}+\Delta t^{-1} \int_{t_{n-1}}^{t_{n}}\left(\left\|A_{h} \mathbf{u}_{h}\right\|_{0}^{2}+\left\|\mathbf{u}_{h s}\right\|_{0}^{2}\right) d s\right) .
$$

Noting $\widehat{d}_{n}^{\prime}=\frac{1}{2} \widehat{b}_{n}^{\prime}$, by Theorems 2.2, 3.2 and 4.1, we have

$$
\Delta t \sum_{n=1}^{N} \widehat{d}_{n} \leq C .
$$

Using Theorems 4.1, 4.2 and summing (4.14) from $n=3$ to $m$, we obtain

$$
\begin{aligned}
& \left\|e_{\mathbf{u}}^{m}\right\|_{-2}^{2}+\frac{v}{2}\left\|e_{\mathbf{u}}^{m}\right\|_{-1}^{2} \Delta t+\Delta t \sum_{n=3}^{m}\left(\frac{1}{2}\left\|d_{t} e_{\mathbf{u}}^{n}\right\|_{-2}^{2} \Delta t+v\left\|\bar{e}_{\mathbf{u}}^{n}\right\|_{-1}^{2}\right) \\
\leq & \Delta t \sum_{n=2}^{m-1} \widehat{d}_{n}\left(\left\|e_{\mathbf{u}}^{n}\right\|_{-1}^{2}+\frac{v}{2}\left\|e_{\mathbf{u}}^{n}\right\|_{0}^{2} \Delta t\right)+C \Delta t^{3} .
\end{aligned}
$$

Secondly, choosing $\psi_{h}=2 A_{h}^{-2} e_{T}^{n} \Delta t \in W_{h}, \mathbf{v}_{h}=0, q_{h}=0$ in (4.2), it yields

$$
\begin{aligned}
\left\|e_{T}^{n}\right\|_{-2}^{2}-\left\|e_{T}^{n-1}\right\|_{-2}^{2}+\left\|d_{t} e_{T}^{n}\right\|_{-2}^{2} \Delta t^{2}+\frac{\operatorname{Pr}^{-1} v}{2}\left(\left\|e_{T}^{n}\right\|_{-1}^{2}-\left\|e_{T}^{n-1}\right\|_{-1}^{2}\right) \Delta t+2 \operatorname{Pr}^{-1} v\left\|\bar{e}_{T}^{n}\right\|_{-1}^{2} \Delta t \\
\quad+2 \tilde{b}\left(e_{\mathbf{u}}^{n-1}, T_{h}\left(t_{n-1}\right), A_{h}^{-2} e_{T}^{n}\right) \Delta t+2 \tilde{b}\left(\mathbf{u}_{h}\left(t_{n-1}\right), e_{T}^{n-1}, A_{h}^{-2} e_{T}^{n}\right) \Delta t \\
\quad-2 \tilde{b}\left(\bar{e}_{\mathbf{u}}^{n-1}, \bar{e}_{T}^{n-1}, A_{h}^{-2} e_{T}^{n}\right) \Delta t-\tilde{b}\left(\bar{e}_{\mathbf{u}}^{n-1}, d_{t} e_{T}^{n-1}, A_{h}^{-2} e_{T}^{n}\right) \Delta t^{2} \\
\quad-\tilde{b}\left(d_{t} e_{\mathbf{u}}^{n-1}, \bar{e}_{T}^{n-1}, A_{h}^{-2} e_{T}^{n}\right) \Delta t^{2}-\frac{1}{2} \tilde{b}\left(d_{t} e_{\mathbf{u}}^{n-1}, d_{t} e_{T}^{n-1}, A_{h}^{-2} e_{T}^{n}\right) \Delta t^{3} \\
=2\left(e_{n}^{\prime}, A_{h}^{-2} e_{T}^{n}\right) \Delta t .
\end{aligned}
$$

Using Lemma 2.2, the bilinear term properties and (2.2b), we have

$$
\begin{aligned}
& 2\left|\tilde{b}\left(e_{\mathbf{u}}^{n-1}, T_{h}\left(t_{n-1}\right), A_{h}^{-2} d_{t} e_{T}^{n}\right)\right| \Delta t^{2}+2\left|\tilde{b}\left(\mathbf{u}_{h}\left(t_{n-1}\right), e_{T}^{n-1}, A_{h}^{-2} d_{t} e_{T}^{n}\right)\right| \Delta t^{2} \\
\leq & \frac{1}{16}\left\|d_{t} e_{T}^{n}\right\|_{-2}^{2} \Delta t^{2}+c\left\|A_{h} \mathbf{u}_{h}\left(t_{n-1}\right)\right\|_{0}^{2}\left\|e_{T}^{n-1}\right\|_{-1}^{2} \Delta t^{2}+c\left\|A_{h} T_{h}\left(t_{n-1}\right)\right\|_{0}^{2}\left\|e_{\mathbf{u}}^{n-1}\right\|_{-1}^{2} \Delta t^{2},
\end{aligned}
$$


$2\left|\tilde{b}\left(\mathbf{u}_{h}\left(t_{n-1}\right), e_{T}^{n-1}, A_{h}^{-2} e_{T}^{n-1}\right)\right| \Delta t$

$\leq \frac{P r^{-1} v}{8}\left\|\bar{e}_{T}^{n}\right\|_{-1}^{2} \Delta t+\frac{1}{8}\left\|d_{t} e_{T}^{n}\right\|_{-1}^{2} \Delta t^{3}+c\left\|A_{h} \mathbf{u}_{h}\left(t_{n-1}\right)\right\|_{0}^{2}\left\|e_{T}^{n-1}\right\|_{-2}^{2} \Delta t$,

$2\left|\tilde{b}\left(e_{\mathbf{u}}^{n-1}, T_{h}\left(t_{n-1}\right), A_{h}^{-2} e_{T}^{n-1}\right)\right| \Delta t$

$\leq \frac{P r^{-1} v}{8}\left\|\bar{e}_{\mathbf{u}}^{n}\right\|_{-1}^{2} \Delta t+\frac{1}{8}\left\|d_{t} e_{\mathbf{u}}^{n}\right\|_{-1}^{2} \Delta t^{3}+c\left\|A_{h} T_{h}\left(t_{n-1}\right)\right\|_{0}^{2}\left\|e_{T}^{n-1}\right\|_{-2}^{2} \Delta t$,

$2\left|\tilde{b}\left(\bar{e}_{\mathbf{u}}^{n-1}, \bar{e}_{T}^{n-1}, A_{h}^{-2} d_{t} e_{T}^{n}\right)\right| \Delta t^{2} \leq \frac{1}{16}\left\|d_{t} e_{T}^{n}\right\|_{-2}^{2} \Delta t^{2}+c\left\|\bar{e}_{\mathbf{u}}^{n-1}\right\|_{0}^{2}\left\|\bar{e}_{T}^{n-1}\right\|_{1}^{2} \Delta t^{2}$,

$2\left|\tilde{b}\left(\bar{e}_{\mathbf{u}}^{n-1}, \bar{e}_{T}^{n-1}, A_{h}^{-2} e_{T}^{n-1}\right)\right| \Delta t \leq \frac{P r^{-1} v}{8}\left\|\bar{e}_{T}^{n-1}\right\|_{-1}^{2} \Delta t+c\left\|A_{h} \bar{e}_{\mathbf{u}}^{n-1}\right\|_{0}^{2}\left\|e_{T}^{n-1}\right\|_{-2}^{2} \Delta t$,

$\left|\tilde{b}\left(d_{t} e_{\mathbf{u}}^{n-1}, \bar{e}_{T}^{n-1}, A_{h}^{-2} d_{t} e_{T}^{n}\right)\right| \Delta t^{3}+\left|\tilde{b}\left(\bar{e}_{\mathbf{u}}^{n-1}, d_{t} e_{T}^{n-1}, A_{h}^{-2} d_{t} e_{T}^{n}\right)\right| \Delta t^{3}$

$\leq \frac{1}{16}\left\|d_{t} e_{T}^{n}\right\|_{-2}^{2} \Delta t^{2}+c\left\|A_{h} \bar{e}_{T}^{n-1}\right\|_{0}^{2}\left\|e_{\mathbf{u}}^{n-1}-e_{\mathbf{u}}^{n-2}\right\|_{-1}^{2} \Delta t^{2}+c\left\|A_{h} \bar{e}_{\mathbf{u}}^{n-1}\right\|_{0}^{2}\left\|e_{T}^{n-1}-e_{T}^{n-2}\right\|_{-1}^{2} \Delta t^{2}$,

$\left|\tilde{b}\left(d_{t} e_{\mathbf{u}}^{n-1}, \bar{e}_{T}^{n-1}, A_{h}^{-2} e_{T}^{n-1}\right)\right| \Delta t^{2} \leq \frac{1}{8}\left\|d_{t} e_{\mathbf{u}}^{n-1}\right\|_{-1}^{2} \Delta t^{3}+c\left\|A_{h} \bar{e}_{T}^{n-1}\right\|_{0}^{2}\left\|e_{T}^{n-1}\right\|_{-2}^{2} \Delta t$,

$\left|\tilde{b}\left(\bar{e}_{\mathbf{u}}^{n-1}, d_{t} e_{T}^{n-1}, A_{h}^{-2} e_{T}^{n-1}\right)\right| \Delta t^{2} \leq \frac{1}{8}\left\|d_{t} e_{T}^{n-1}\right\|_{-1}^{2} \Delta t^{3}+c\left\|A_{h} \bar{e}_{\mathbf{u}}^{n-1}\right\|_{0}^{2}\left\|e_{T}^{n-1}\right\|_{-2}^{2} \Delta t$,

$\frac{1}{2}\left|\tilde{b}\left(d_{t} e_{\mathbf{u}}^{n-1}, d_{t} e_{T}^{n-1}, A_{h}^{-2} d_{t} e_{T}^{n}\right)\right| \Delta t^{4} \leq \frac{1}{16}\left\|d_{t} e_{T}^{n}\right\|_{-2}^{2} \Delta t^{2}+c\left\|e_{T}^{n-1}-e_{T}^{n-2}\right\|_{1}^{2}\left\|d_{t} e_{\mathbf{u}}^{n-1}\right\|_{0}^{2} \Delta t^{4}$,

$\frac{1}{2}\left|\tilde{b}\left(d_{t} e_{\mathbf{u}}^{n-1}, d_{t} e_{T}^{n-1}, A_{h}^{-2} e_{T}^{n-1}\right)\right| \Delta t^{3} \leq \frac{1}{8}\left\|d_{t} e_{T}^{n-1}\right\|_{0}^{2} \Delta t^{4}+c\left\|d_{t} e_{\mathbf{u}}^{n-1}\right\|_{1}^{2}\left\|e_{T}^{n-1}\right\|_{-2}^{2} \Delta t^{2}$,

$\int_{t_{n-1}}^{t_{n}}\left(s-t_{n-1}\right)\left(t_{n}-s\right)\left(g_{s s}, A_{h}^{-2}\left(d_{t} e_{T}^{n} \Delta t+e_{T}^{n-1}\right)\right) d s$

$\leq \frac{1}{16}\left\|d_{t} e_{T}^{n}\right\|_{-2}^{2} \Delta t^{2}+\left\|e_{T}^{n-1}\right\|_{-2}^{2} \Delta t+c \Delta t^{3} \int_{t_{n-1}}^{t_{n}}\left(\left\|g_{s s}\right\|_{0}^{2}+\Delta t\left\|g_{s s}\right\|_{0}^{2}\right) d s$,

$\int_{t_{n-1}}^{t_{n}}\left(s-t_{n-1}\right)\left(t_{n}-s\right) \tilde{a}\left(T_{h s s}(s), A_{h}^{-2}\left(\frac{1}{2} d_{t} e_{T}^{n} \Delta t+\bar{e}_{T}^{n}\right)\right) d s$

$\leq \frac{1}{16}\left\|d_{t} e_{T}^{n}\right\|_{-2}^{2} \Delta t^{2}+\frac{P r^{-1} v}{8}\left\|\bar{e}_{T}^{n}\right\|_{-1}^{2} \Delta t+c \Delta t^{3} \int_{t_{n-1}}^{t_{n}}\left(\left\|T_{h s s}\right\|_{0}^{2}+\Delta t\left\|T_{h s s}\right\|_{-1}^{2}\right) d s$,

$\Delta t^{2} \int_{t_{n-1}}^{t_{n}}\left|\tilde{b}_{s s}\left(\mathbf{u}_{h}(s), T_{h}(s), A_{h}^{-2}\left(d_{t} e_{T}^{n} \Delta t+e_{T}^{n-1}\right)\right)\right| d s$

$\leq \frac{1}{16}\left\|d_{t} e_{T}^{n}\right\|_{-2}^{2} \Delta t^{2}+c \int_{t_{n-1}}^{t_{n}}\left(\left\|A_{h} T_{h}\right\|_{0}^{2}+\left\|T_{h s}\right\|_{0}^{2}\right) d s\left\|e_{T}^{n-1}\right\|_{-2}^{2}$

$+c \Delta t^{3} \int_{t_{n-1}}^{t_{n}}\left[\left(\Delta t+\Delta t^{2}\left\|A_{h} T_{h}\right\|_{0}^{2}\right)\left\|\mathbf{u}_{h s s}\right\|_{-1}^{2}+\left(\Delta t+\Delta t^{2}\left\|T_{h s}\right\|_{0}^{2}\right)\left\|\mathbf{u}_{h s}\right\|_{1}^{2}\right] d s$,

$\Delta t^{2} \int_{t_{n-1}}^{t_{n}}\left|\tilde{b}_{s}\left(\mathbf{u}_{h}(s), T_{h}(s), A_{h}^{-2}\left(d_{t} e_{T}^{n} \Delta t+e_{T}^{n-1}\right)\right)\right| d s$

$\leq \frac{1}{16}\left\|d_{t} e_{T}^{n}\right\|_{-2}^{2} \Delta t^{2}+c \int_{t_{n-1}}^{t_{n}}\left(\left\|A_{h} T_{h}\right\|_{0}^{2}+\left\|T_{h}\right\|_{0}^{2}\right) d s\left\|e_{T}^{n-1}\right\|_{-2}^{2}$

$+c \Delta t^{3} \int_{t_{n-1}}^{t_{n}}\left[\left(\Delta t+\Delta t^{2}\left\|A_{h} T_{h}\right\|_{0}^{2}\right)\left\|\mathbf{u}_{h s}\right\|_{-1}^{2}+\left(\Delta t+\Delta t^{2}\left\|T_{h s}\right\|_{0}^{2}\right)\left\|\mathbf{u}_{h}\right\|_{1}^{2}\right] d s$. 
Combining above inequalities with (4.17), for all $3 \leq n \leq N$, we obtain

$$
\begin{aligned}
& \left(\left\|e_{T}^{n}\right\|_{-2}^{2}+\frac{P r^{-1} v}{2}\left\|e_{T}^{n}\right\|_{-1}^{2} \Delta t\right)-\left(\left\|e_{T}^{n-1}\right\|_{-2}^{2}+\frac{P^{-1} v}{2}\left\|e_{T}^{n-1}\right\|_{-1}^{2} \Delta t\right) \\
& \quad+\frac{1}{2}\left\|d_{t} e_{T}^{n}\right\|_{-2}^{2} \Delta t^{2}+\frac{3 P r^{-1} v}{2}\left\|\bar{e}_{T}^{n}\right\|_{-1}^{2} \Delta t-\frac{P^{-1} v}{2}\left\|e_{T}^{n-1}\right\|_{-1}^{2} \Delta t \\
& \leq \frac{1}{2} \widetilde{b}_{n-1}^{\prime}\left(\left\|e_{T}^{n-1}\right\|_{-2}^{2}+\frac{P r^{-1} v}{2}\left\|e_{T}^{n-1}\right\|_{-1}^{2} \Delta t\right) \Delta t+\frac{1}{2} \widetilde{c}_{n-2}\left(\left\|e_{T}^{n-2}\right\|_{-2}^{2}+\frac{P^{-1} v}{2}\left\|e_{T}^{n-2}\right\|_{-1}^{2} \Delta t\right) \Delta t \\
& \quad+c\left\|\bar{e}_{\mathbf{u}}^{n-1}\right\|_{0}^{2}\left\|\bar{e}_{T}^{n-1}\right\|_{1}^{2} \Delta t^{2}+c\left\|A_{h} \bar{e}_{\mathbf{u}}^{n-1}\right\|_{0}^{2}\left\|e_{T}^{n-1}-e_{T}^{n-2}\right\|_{-1}^{2} \Delta t^{2}+c P r^{-1} v\left\|_{e_{\mathbf{u}}^{n}}\right\|_{-1}^{2} \\
& \quad+c\left\|A_{h} e_{T}^{n-1}\right\|_{0}^{2}\left\|e_{\mathbf{u}}^{n-1}-e_{\mathbf{u}}^{n-2}\right\|_{-1}^{2} \Delta t^{2}+c\left\|d_{t} e_{\mathbf{u}}^{n-1}\right\|_{-1}^{2} \Delta t^{3}+c\left\|_{t} e_{\mathbf{u}}^{n}\right\|_{-1}^{2} \Delta t^{3} \\
& \quad+c\left\|d_{t} e_{T}^{n-1}\right\|_{0}^{2} \Delta t^{4}+c\left\|A_{h} T_{h}\left(t_{n-1}\right)\right\|_{0}^{2}\left\|e_{\mathbf{u}}^{n-1}\right\|_{-1}^{2} \Delta t^{2} \\
& \quad+c\left\|e_{T}^{n-1}-e_{T}^{n-2}\right\|_{1}^{2}\left\|d_{t} e_{\mathbf{u}}^{n-1}\right\|_{0}^{2} \Delta t^{4}+c\left\|d_{t} e_{T}^{n-1}\right\|_{-1}^{2} \Delta t^{3}+c\left\|d_{t} e_{T}^{n}\right\|_{-1}^{2} \Delta t^{3} \\
& \quad+c \Delta t^{3} \int_{t_{n-1}}^{t_{n}}\left(\left\|g_{s s}\right\|_{0}^{2}+\Delta t\left\|g_{s s}\right\|_{0}^{2}\right) d s+c \Delta t^{3} \int_{t_{n-1}}^{t_{n}}\left(\left\|T_{h s s}\right\|_{0}^{2}+\Delta t\left\|T_{h s s}\right\|_{-1}^{2}\right) d s \\
& \quad+c \Delta t^{3} \int_{t_{n-1}}^{t_{n}}\left[\left(\Delta t+\Delta t^{2}\left\|A_{h} T_{h}\right\|_{0}^{2}\right)\left\|\mathbf{u}_{h s s}\right\|_{-1}^{2}+\left(\Delta t+\Delta t^{2}\left\|T_{h s}\right\|_{0}^{2}\right)\left\|\mathbf{u}_{h s}\right\|_{1}^{2}\right] d s, \\
& \quad+c \Delta t^{3} \int_{t_{n-1}}^{t_{n}}\left[\left(\Delta t+\Delta t^{2}\left\|A_{h} T_{h}\right\|_{0}^{2}\right)\left\|\mathbf{u}_{h s}\right\|_{-1}^{2}+\left(\Delta t+\Delta t^{2}\left\|T_{h s}\right\|_{0}^{2}\right)\left\|\mathbf{u}_{h}\right\|_{1}^{2}\right] d s,
\end{aligned}
$$

where $\widetilde{c}_{n-2}=c\left\|A_{h} \mathbf{u}_{h}\left(t_{n-2}\right)\right\|_{0}^{2}$,

$$
\begin{aligned}
\widetilde{b}_{n-1}^{\prime}=c & \left(\left\|d_{t} e_{\mathbf{u}}^{n-1}\right\|_{1}^{2} \Delta t+\left\|A_{h} \mathbf{u}_{h}\left(t_{n-1}\right)\right\|_{0}^{2}+\left\|A_{h} \bar{e}_{T}^{n-1}\right\|_{0}^{2}+\left\|A_{h} \bar{e}_{\mathbf{u}}^{n-1}\right\|_{0}^{2}\right. \\
& \left.+\int_{t_{n-1}}^{t_{n}}\left(\left\|A_{h} T_{h}\right\|_{0}^{2}+\left\|T_{h s}\right\|_{0}^{2}+\left\|T_{h}\right\|_{0}^{2}\right) d s+\left\|A_{h} T_{h}\left(t_{n-1}\right)\right\|_{0}^{2}\right) .
\end{aligned}
$$

With Theorems $2.2,3.2,4.1$ and noting the fact that $\widetilde{d}_{n}^{\prime}=\frac{1}{2}\left(\widetilde{b}_{n}^{\prime}+\widetilde{c}_{n}\right)$, we have

$$
\Delta t \sum_{n=1}^{N} \widetilde{d}_{n}^{\prime} \leq C .
$$

Summing (4.18) from $n=3$ to $m$, using Theorems 4.1 and 4.2 , we get

$$
\begin{aligned}
& \left\|e_{T}^{m}\right\|_{-2}^{2}+\frac{\operatorname{Pr}^{-1} v}{2}\left\|e_{T}^{m}\right\|_{-1}^{2} \Delta t+\Delta t \sum_{n=3}^{m}\left(\frac{1}{2}\left\|d_{t} e_{T}^{n}\right\|_{-2}^{2} \Delta t+\operatorname{Pr}^{-1} v\left\|\bar{e}_{T}^{n}\right\|_{-1}^{2}\right) \\
\leq & \Delta t \sum_{n=2}^{m-1} \widetilde{d}_{n}^{\prime}\left(\left\|e_{T}^{n}\right\|_{-1}^{2}+\frac{P^{-1} v}{2}\left\|e_{T}^{n}\right\|_{0}^{2} \Delta t\right)+C \Delta t^{3} .
\end{aligned}
$$

Using (4.19) and applying Lemma 2.2 to (4.16) and (4.20), we complete the proof.

Theorem 4.3. Under the assumptions of (A1)-(A3) and (3.9), for all $1 \leq m \leq N$, we have

$$
\sigma^{2}\left(t_{m}\right)\left\|e_{u}^{m}, e_{T}^{m}\right\|_{0}^{2}+\min \left\{v, \operatorname{Pr}^{-1} v\right\} \Delta t \sum_{n=1}^{m} \sigma^{2}\left(t_{n}\right)\left\|\bar{e}_{u}^{n}, \bar{e}_{T}^{n}\right\|_{1}^{2} \leq C \Delta t^{3} .
$$


Proof. For all $3 \leq m \leq N$, summing from $n=3$ to $m$, using Theorems 2.2, 4.1, 4.2, 4.3, and multiplying by $\sigma\left(t_{n}\right)$ in (4.6), we get

$$
\begin{aligned}
& \quad \sigma\left(t_{m}\right)\left(\left\|e_{\mathbf{u}}^{m}\right\|_{-1}^{2}+\frac{v}{2}\left\|e_{\mathbf{u}}^{m}\right\|_{0}^{2} \Delta t\right)+\Delta t \sum_{n=3}^{m} \sigma\left(t_{n}\right)\left(\frac{1}{2}\left\|d_{t} e_{\mathbf{u}}^{n}\right\|_{-1}^{2} \Delta t+v\left\|\bar{e}_{\mathbf{u}}^{n}\right\|_{0}^{2}\right) \\
& \leq \Delta t \sum_{n=2}^{m-1}\left(\left\|\bar{e}_{\mathbf{u}}^{n}+\frac{1}{2} d_{t} e_{\mathbf{u}}^{n} \Delta t\right\|_{-1}^{2}+\frac{v}{2}\left\|\bar{e}_{\mathbf{u}}^{n}+\frac{1}{2} d_{t} e_{\mathbf{u}}^{n} \Delta t\right\|_{0}^{2} \Delta t\right)+C \Delta t^{3} \\
& \quad+2 \Delta t \sum_{n=2}^{m-1} \widehat{d}_{n} \sigma\left(t_{n}\right)\left(\left\|e_{\mathbf{u}}^{n}\right\|_{-1}^{2}+\frac{v}{2}\left\|e_{\mathbf{u}}^{n}\right\|_{0}^{2} \Delta t\right) \\
& \leq C \Delta t^{3}+2 \Delta t \sum_{n=2}^{m-1} \widehat{d}_{n} \sigma\left(t_{n}\right)\left(\left\|e_{\mathbf{u}}^{n}\right\|_{-1}^{2}+\frac{v}{2}\left\|e_{\mathbf{u}}^{n}\right\|_{0}^{2} \Delta t\right) .
\end{aligned}
$$

For all $1 \leq m \leq N$, applying Lemma 2.2 to (4.21) and using (4.7), we have

$$
\sigma\left(t_{m}\right)\left(\left\|e_{\mathbf{u}}^{m}\right\|_{-1}^{2}+\frac{v}{2}\left\|e_{\mathbf{u}}^{m}\right\|_{0}^{2} \Delta t\right)+\Delta t \sum_{n=3}^{m} \sigma\left(t_{n}\right)\left(\frac{1}{2}\left\|d_{t} e_{\mathbf{u}}^{n}\right\|_{-1}^{2} \Delta t+v\left\|\bar{e}_{\mathbf{u}}^{n}\right\|_{0}^{2}\right) \leq C \Delta t^{3}
$$

Multiplying by $\sigma\left(t_{n}\right)$ in (4.10) with $\alpha=-1$. Summing from $n=3$ to $m$ with $3 \leq m \leq N$, we get

$$
\begin{aligned}
& \quad \sigma\left(t_{m}\right)\left(\left\|e_{T}^{m}\right\|_{-1}^{2}+\frac{P^{-1} v}{2}\left\|e_{T}^{m}\right\|_{0}^{2} \Delta t\right)+\Delta t \sum_{n=3}^{m} \sigma\left(t_{n}\right)\left(\frac{1}{2}\left\|d_{t} e_{T}^{n}\right\|_{-1}^{2} \Delta t+\operatorname{Pr}^{-1} v\left\|\bar{e}_{T}^{n}\right\|_{0}^{2}\right) \\
& \leq \Delta t \sum_{n=2}^{m-1}\left(\left\|\bar{e}_{T}^{n}+\frac{1}{2} d_{t} e_{T}^{n} \Delta t\right\|_{-1}^{2}+\frac{P r^{-1} v}{2}\left\|\bar{e}_{T}^{n}+\frac{1}{2} d_{t} e_{T}^{n} \Delta t\right\|_{0}^{2} \Delta t\right)+C \Delta t^{3} \\
& \quad+2 \Delta t \sum_{n=2}^{m-1} \widetilde{d}_{n} \sigma\left(t_{n}\right)\left(\left\|e_{T}^{n}\right\|_{-1}^{2}+\frac{\operatorname{Pr}^{-1} v}{2}\left\|e_{T}^{n}\right\|_{0}^{2} \Delta t\right) \\
& \leq C \Delta t^{3}+2 \Delta t \sum_{n=2}^{m-1} \widetilde{d}_{n} \sigma\left(t_{n}\right)\left(\left\|e_{T}^{n}\right\|_{-1}^{2}+\frac{\operatorname{Pr}^{-1} v}{2}\left\|e_{T}^{n}\right\|_{0}^{2} \Delta t\right)
\end{aligned}
$$

Applying Lemma 2.1 to (4.23) and using (4.11), for $1 \leq m \leq N$, we get

$$
\begin{aligned}
& \sigma\left(t_{m}\right)\left(\left\|e_{T}^{m}\right\|_{-1}^{2}+\frac{\operatorname{Pr}^{-1} v}{2}\left\|e_{T}^{m}\right\|_{0}^{2} \Delta t\right) \\
& +\Delta t \sum_{n=3}^{m} \sigma\left(t_{n}\right)\left(\frac{1}{2}\left\|d_{t} e_{T}^{n}\right\|_{-1}^{2} \Delta t+\operatorname{Pr}^{-1} v\left\|\bar{e}_{T}^{n}\right\|_{0}^{2}\right) \leq C \Delta t^{3}
\end{aligned}
$$

Thanks to (4.22), multiplying by $\sigma\left(t_{n}\right)$ in (4.5) with $\alpha=0$, summing from $n=3$ to $m$ with 
$3 \leq m \leq N$, we obtain

$$
\begin{aligned}
& \sigma\left(t_{m}\right)\left(\left\|e_{\mathbf{u}}^{m}\right\|_{0}^{2}+\frac{v}{2}\left\|e_{\mathbf{u}}^{m}\right\|_{1}^{2} \Delta t\right)+\Delta t \sum_{n=3}^{m} \sigma\left(t_{n}\right)\left(\frac{1}{2}\left\|d_{t} e_{\mathbf{u}}^{n}\right\|_{0}^{2} \Delta t+v\left\|\bar{e}_{\mathbf{u}}^{n}\right\|_{1}^{2}\right) \\
\leq & \Delta t \sum_{n=2}^{m-1}\left(\left\|\bar{e}_{\mathbf{u}}^{n}+\frac{1}{2} d_{t} e_{\mathbf{u}}^{n} \Delta t\right\|_{0}^{2}+\frac{v}{2}\left\|\bar{e}_{\mathbf{u}}^{n}+\frac{1}{2} d_{t} e_{\mathbf{u}}^{n} \Delta t\right\|_{1}^{2} \Delta t\right)+C \Delta t^{2} \\
& +2 \Delta t \sum_{n=2}^{m-1} \widehat{d}_{n} \sigma\left(t_{n}\right)\left(\left\|e_{\mathbf{u}}^{n}\right\|_{0}^{2}+\frac{v}{2}\left\|e_{\mathbf{u}}^{n}\right\|_{1}^{2} \Delta t\right) \\
\leq & C \Delta t^{2}+2 \Delta t \sum_{n=2}^{m-1} \widehat{d_{n}} \sigma\left(t_{n}\right)\left(\left\|e_{\mathbf{u}}^{n}\right\|_{0}^{2}+\frac{v}{2}\left\|e_{\mathbf{u}}^{n}\right\|_{1}^{2} \Delta t\right) .
\end{aligned}
$$

For all $1 \leq m \leq N$, by using Lemma 2.1 to (4.25) and (4.7), we have

$$
\sigma\left(t_{m}\right)\left(\left\|e_{\mathbf{u}}^{m}\right\|_{0}^{2}+\frac{v}{2}\left\|e_{\mathbf{u}}^{m}\right\|_{1}^{2} \Delta t\right)+\Delta t \sum_{n=3}^{m} \sigma\left(t_{n}\right)\left(\frac{1}{2}\left\|d_{t} e_{\mathbf{u}}^{n}\right\|_{0}^{2} \Delta t+v\left\|\bar{e}_{\mathbf{u}}^{n}\right\|_{1}^{2}\right) \leq C \Delta t^{2} .
$$

For all $3 \leq m \leq N$, applying (4.24), Theorems 2.2, 4.1, 4.2, 4.3, multiplying $\sigma\left(t_{n}\right)$ in (4.9) with $\alpha=0$ and summing from $n=3$ to $m$, one finds

$$
\begin{aligned}
& \sigma\left(t_{m}\right)\left(\left\|e_{T}^{m}\right\|_{0}^{2}+\frac{P^{-1} v}{2}\left\|e_{T}^{m}\right\|_{1}^{2} \Delta t\right)+\Delta t \sum_{n=3}^{m} \sigma\left(t_{n}\right)\left(\frac{1}{2}\left\|d_{t} e_{T}^{n}\right\|_{0}^{2} \Delta t+\operatorname{Pr}^{-1} v\left\|_{e_{T}^{n}}^{n}\right\|_{1}^{2}\right) \\
\leq & \Delta t \sum_{n=2}^{m-1}\left(\left\|\bar{e}_{T}^{n}+\frac{1}{2} d_{t} e_{T}^{n} \Delta t\right\|_{0}^{2}+\frac{P r^{-1} v}{2}\left\|\bar{e}_{T}^{n}+\frac{1}{2} d_{t} e_{T}^{n} \Delta t\right\|_{1}^{2} \Delta t\right)+C \Delta t^{2} \\
& +2 \Delta t \sum_{n=2}^{m-1} \widetilde{d}_{n} \sigma\left(t_{n}\right)\left(\left\|e_{T}^{n}\right\|_{0}^{2}+\frac{P r^{-1} v}{2}\left\|e_{T}^{n}\right\|_{1}^{2} \Delta t\right) \\
\leq & C \Delta t^{2}+2 \Delta t \sum_{n=2}^{m-1} \widetilde{d}_{n} \sigma\left(t_{n}\right)\left(\left\|e_{T}^{n}\right\|_{0}^{2}+\frac{P r^{-1} v}{2}\left\|e_{T}^{n}\right\|_{1}^{2} \Delta t\right) .
\end{aligned}
$$

For all $1 \leq m \leq N$, thanks to (4.11) and using Lemma 2.1 to (4.27), we have

$$
\sigma\left(t_{m}\right)\left(\left\|e_{T}^{m}\right\|_{0}^{2}+\frac{P r^{-1} v}{2}\left\|e_{T}^{m}\right\|_{1}^{2} \Delta t\right)+\Delta t \sum_{n=3}^{m} \sigma\left(t_{n}\right)\left(\frac{1}{2}\left\|d_{t} e_{T}^{n}\right\|_{0}^{2} \Delta t+P r^{-1} v\left\|\bar{e}_{T}^{n}\right\|_{1}^{2}\right) \leq C \Delta t^{2} .
$$

Secondly, taking $\mathbf{v}_{h}=2 \bar{e}_{\mathbf{u}}^{n} \Delta t \in V_{h}, q_{h}=0, \psi_{h}=0$ in (4.2), we get

$$
\begin{aligned}
& \left\|e_{\mathbf{u}}^{n}\right\|_{0}^{2}-\left\|e_{\mathbf{u}}^{n-1}\right\|_{0}^{2}+2 v\left\|\bar{e}_{\mathbf{u}}^{n}\right\|_{1}^{2} \Delta t+2 b\left(e_{\mathbf{u}}^{n-1}, \mathbf{u}_{h}\left(t_{n-1}\right), \bar{e}_{\mathbf{u}}^{n}\right) \Delta t+2 b\left(\mathbf{u}_{h}\left(t_{n-1}\right), e_{\mathbf{u}}^{n-1}, \bar{e}_{\mathbf{u}}^{n}\right) \Delta t \\
& \quad-2 b\left(\bar{e}_{\mathbf{u}}^{n-1}, \bar{e}_{\mathbf{u}}^{n-1}, \bar{e}_{\mathbf{u}}^{n}\right) \Delta t-b\left(d_{t} e_{\mathbf{u}}^{n-1}, \bar{e}_{\mathbf{u}}^{n-1}, \bar{e}_{\mathbf{u}}^{n}\right) \Delta t^{2} \\
& \quad-b\left(\bar{e}_{\mathbf{u}}^{n-1}, d_{t} e_{\mathbf{u}}^{n-1}, \bar{e}_{\mathbf{u}}^{n}\right) \Delta t^{2}-\frac{1}{2} b\left(d_{t} e_{\mathbf{u}}^{n-1}, d_{t} e_{\mathbf{u}}^{n-1}, \bar{e}_{\mathbf{u}}^{n}\right) \Delta t^{3}=2\left(e_{n}, \bar{e}_{\mathbf{u}}^{n}\right) \Delta t
\end{aligned}
$$


For all $3 \leq n \leq N$, by the similar proof as Theorem 4.2 , we obtain

$$
\begin{aligned}
& \left\|e_{\mathbf{u}}^{n}\right\|_{0}^{2}-\left\|e_{\mathbf{u}}^{n-1}\right\|_{0}^{2}+v\left\|\bar{e}_{\mathbf{u}}^{n}\right\|_{1}^{2} \Delta t \\
& \leq \frac{1}{2} b_{n-1}\left\|e_{\mathbf{u}}^{n-1}\right\|_{0}^{2} \Delta t+\frac{1}{2} c_{n-2}\left\|e_{\mathbf{u}}^{n-2}\right\|_{0}^{2} \Delta t \\
& \quad+c\left\|e_{\mathbf{u}}^{n-1}-e_{\mathbf{u}}^{n-2}\right\|_{1}^{4}\left\|e_{\mathbf{u}}^{n-1}-e_{\mathbf{u}}^{n-2}\right\|_{1}^{2} \Delta t^{2}+8 v^{-1}\left\|A_{h}^{-1 / 2} P_{h} e_{n}\right\|_{0}^{2} \Delta t .
\end{aligned}
$$

Thanks to (4.22), (4.26), Theorems $2.2,4.1,4.2,4.3$, and multiplying by $\sigma^{2}\left(t_{n}\right)$ in above inequality, summing from $n=3$ to $m$ for all $3 \leq m \leq N$, one gets

$$
\begin{aligned}
& \quad \sigma^{2}\left(t_{m}\right)\left\|e_{\mathbf{u}}^{m}\right\|_{0}^{2}+v \Delta t \sum_{n=3}^{m} \sigma^{2}\left(t_{n}\right)\left\|\bar{e}_{\mathbf{u}}^{n}\right\|_{1}^{2} \\
& \leq 3 \Delta t \sum_{n=2}^{m-1} \sigma\left(t_{n}\right)\left\|\bar{e}_{\mathbf{u}}^{n}+\frac{1}{2} d_{t} e_{\mathbf{u}}^{n} \Delta t\right\|_{0}^{2}+4 \Delta t \sum_{n=2}^{m-1} \widehat{d}_{n} \sigma^{2}\left(t_{n}\right)\left\|e_{\mathbf{u}}^{n}\right\|_{0}^{2} \\
& \quad+c \Delta t \sum_{n=3}^{m} \sigma^{2}\left(t_{n}\right)\left[\left\|A_{h}^{-1 / 2} P_{h} e_{n}\right\|_{0}^{2}+\left\|e_{\mathbf{u}}^{n-1}-e_{\mathbf{u}}^{n-2}\right\|_{1}^{4}\left\|e_{\mathbf{u}}^{n-1}-e_{\mathbf{u}}^{n-2}\right\|_{1}^{2} \Delta t\right] \\
& \leq C \Delta t^{3}+4 \Delta t \sum_{n=2}^{m-1} \widehat{d}_{n} \sigma^{2}\left(t_{n}\right)\left\|e_{\mathbf{u}}^{n}\right\|_{0}^{2} .
\end{aligned}
$$

In the same way, taking $\psi_{h}=2 \bar{e}_{T}^{n} \Delta t \in W_{h}, \mathbf{v}_{h}=0, q_{h}=0$ in (4.2), we have

$$
\begin{aligned}
& \left\|e_{T}^{n}\right\|_{0}^{2}-\left\|e_{T}^{n-1}\right\|_{0}^{2}+2 \operatorname{Pr}^{-1} v\left\|\bar{e}_{T}^{n}\right\|_{1}^{2} \Delta t+2 \tilde{b}\left(e_{\mathbf{u}}^{n-1}, T_{h}\left(t_{n-1}\right), \bar{e}_{T}^{n}\right) \Delta t \\
& \quad+2 \tilde{b}\left(\mathbf{u}_{h}\left(t_{n-1}\right), e_{T}^{n-1}, \bar{e}_{T}^{n}\right) \Delta t-2 \tilde{b}\left(\bar{e}_{\mathbf{u}}^{n-1}, \bar{e}_{T}^{n-1}, \bar{e}_{T}^{n}\right) \Delta t-\tilde{b}\left(d_{t} e_{\mathbf{u}}^{n-1}, \bar{e}_{T}^{n-1}, \bar{e}_{T}^{n}\right) \Delta t^{2} \\
& \quad-\tilde{b}\left(\bar{e}_{\mathbf{u}}^{n-1}, d_{t} e_{T}^{n-1}, \bar{e}_{T}^{n}\right) \Delta t^{2}-\frac{1}{2} \tilde{b}\left(d_{t} e_{\mathbf{u}}^{n-1}, d_{t} e_{T}^{n-1}, \bar{e}_{T}^{n}\right) \Delta t^{3}=2\left(e_{n}^{\prime}, \bar{e}_{T}^{n}\right) \Delta t .
\end{aligned}
$$

For all $3 \leq n \leq N$, by the similar proof as Theorem 4.1 , we obtain

$$
\begin{gathered}
\left\|e_{T}^{n}\right\|_{0}^{2}-\left\|e_{T}^{n-1}\right\|_{0}^{2}+\operatorname{Pr}^{-1} v\left\|e_{T}^{n}\right\|_{1}^{2} \Delta t \\
\leq \frac{1}{2} b_{n-1}\left\|e_{T}^{n-1}\right\|_{0}^{2} \Delta t+c\left\|e_{\mathbf{u}}^{n-1}-e_{\mathbf{u}}^{n-2}\right\|_{1}^{4}\left\|e_{T}^{n-1}-e_{T}^{n-2}\right\|_{1}^{2} \Delta t^{2} \\
+\frac{1}{2} c_{n-2}\left\|e_{T}^{n-2}\right\|_{0}^{2} \Delta t+8 \operatorname{Prv}^{-1}\left\|A_{h}^{-1 / 2} P_{h} e_{n}^{\prime}\right\|_{0}^{2} \Delta t .
\end{gathered}
$$

Using (4.24), (4.28), Theorems 2.2, 4.1, 4.2, 4.3, and multiplying by $\sigma^{2}\left(t_{n}\right)$ in above inequality, summing from $n=3$ to $m$ with $3 \leq m \leq N$, we have

$$
\begin{aligned}
& \sigma^{2}\left(t_{m}\right)\left\|e_{T}^{m}\right\|_{0}^{2}+\operatorname{Pr}^{-1} v \Delta t \sum_{n=3}^{m} \sigma^{2}\left(t_{n}\right)\left\|\bar{e}_{T}^{n}\right\|_{1}^{2} \\
\leq & 3 \Delta t \sum_{n=2}^{m-1} \sigma\left(t_{n}\right)\left\|\bar{e}_{T}^{n}+\frac{1}{2} d_{t} e_{T}^{n} \Delta t\right\|_{0}^{2}+4 \Delta t \sum_{n=2}^{m-1} \widetilde{d}_{n} \sigma^{2}\left(t_{n}\right)\left\|e_{T}^{n}\right\|_{0}^{2}
\end{aligned}
$$




$$
\begin{aligned}
& +c \Delta t \sum_{n=3}^{m} \sigma^{2}\left(t_{n}\right)\left[\left\|A_{h}^{-1 / 2} P_{h} e_{n}^{\prime}\right\|_{0}^{2}+\left\|e_{\mathbf{u}}^{n-1}-e_{\mathbf{u}}^{n-2}\right\|_{1}^{4}\left\|e_{T}^{n-1}-e_{T}^{n-2}\right\|_{1}^{2} \Delta t\right] \\
& \leq C \Delta t^{3}+4 \Delta t \sum_{n=2}^{m-1} \widetilde{d}_{n} \sigma^{2}\left(t_{n}\right)\left\|e_{T}^{n}\right\|_{0}^{2} .
\end{aligned}
$$

Applying (4.7), Lemma 2.1 and combining (4.29) with (4.30), we complete the proof.

Theorem 4.4. Under the assumptions of (A1)-(A3) and (3.9), for all $1 \leq m \leq N$ we have

$$
\begin{aligned}
& \sigma^{2}\left(t_{m}\right)\left\|e_{u}^{m}, e_{T}^{m}\right\|_{1}^{2}+\min \left\{v, \operatorname{Pr}^{-1} v\right\} \Delta t \sum_{n=1}^{m} \sigma^{2}\left(t_{n}\right)\left(\left\|d_{t} e_{u}^{n}, d_{t} e_{T}^{n}\right\|_{0}^{2}\right. \\
& \left.+\left\|A_{h} \bar{e}_{u}^{n}, A_{h} \bar{e}_{T}^{n}\right\|_{0}^{2}\right) \leq C \Delta t^{2} .
\end{aligned}
$$

Proof. For all $3 \leq m \leq N$, using Theorems $2.2,4.1,4.2,4.3$ and multiplying by $\sigma\left(t_{n}\right)$ in (4.5) and (4.9) with $\alpha=1$, summing from $n=3$ to $m$, we have

$$
\begin{aligned}
& \sigma\left(t_{m}\right)\left(\left\|e_{\mathbf{u}}^{m}\right\|_{1}^{2}+\frac{v}{2}\left\|A_{h} e_{\mathbf{u}}^{m}\right\|_{0}^{2} \Delta t\right)+\Delta t \sum_{n=3}^{m} \sigma\left(t_{n}\right)\left(\frac{1}{2}\left\|d_{t} e_{\mathbf{u}}^{n}\right\|_{1}^{2} \Delta t+v\left\|A_{h} \bar{e}_{\mathbf{u}}^{n}\right\|_{0}^{2}\right) \\
& \leq \Delta t \sum_{n=2}^{m-1}\left(\left\|\bar{e}_{\mathbf{u}}^{n}+\frac{1}{2} d_{t} e_{\mathbf{u}}^{n} \Delta t\right\|_{1}^{2}+\frac{v}{2}\left\|A_{h} e_{\mathbf{u}}^{n}\right\|_{0}^{2} \Delta t\right)+C \Delta t+2 \Delta t \sum_{n=2}^{m-1} \widehat{d}_{n} \sigma\left(t_{n}\right)\left(\left\|e_{\mathbf{u}}^{n}\right\|_{1}^{2}+\frac{v}{2}\left\|A_{h} e_{\mathbf{u}}^{n}\right\|_{0}^{2} \Delta t\right) \\
& \leq C \Delta t+2 \Delta t \sum_{n=2}^{m-1} \widehat{d}_{n} \sigma\left(t_{n}\right)\left(\left\|e_{\mathbf{u}}^{n}\right\|_{1}^{2}+\frac{v}{2}\left\|A_{h} e_{\mathbf{u}}^{n}\right\|_{0}^{2} \Delta t\right), \\
& \sigma\left(t_{m}\right)\left(\left\|e_{T}^{m}\right\|_{1}^{2}+\frac{\operatorname{Pr}^{-1} v}{2}\left\|A_{h} e_{T}^{m}\right\|_{0}^{2} \Delta t\right)+\Delta t \sum_{n=3}^{m} \sigma\left(t_{n}\right)\left(\frac{1}{2}\left\|d_{t} e_{T}^{n}\right\|_{1}^{2} \Delta t+\operatorname{Pr}^{-1} v\left\|A_{h} \bar{e}_{T}^{n}\right\|_{0}^{2}\right) \\
& \leq \Delta t \sum_{n=2}^{m-1}\left(\left\|\bar{e}_{T}^{n}+\frac{1}{2} d_{t} e_{T}^{n} \Delta t\right\|_{1}^{2}+\frac{\operatorname{Pr}^{-1} v}{2}\left\|A_{h} e_{T}^{n}\right\|_{0}^{2} \Delta t\right)+C \Delta t \\
& \quad+2 \Delta t \sum_{n=2}^{m-1} \widetilde{d}_{n} \sigma\left(t_{n}\right)\left(\left\|e_{T}^{n}\right\|_{1}^{2}+\frac{\operatorname{Pr}^{-1} v}{2}\left\|A_{h} e_{T}^{n}\right\|_{0}^{2} \Delta t\right) \\
& \leq C \Delta t+2 \Delta t \sum_{n=2}^{m-1} \widetilde{d}_{n} \sigma\left(t_{n}\right)\left(\left\|e_{T}^{n}\right\|_{1}^{2}+\frac{\operatorname{Pr}^{-1} v}{2}\left\|A_{h} e_{T}^{n}\right\|_{0}^{2} \Delta t\right) .
\end{aligned}
$$

For all $1 \leq m \leq N$, thanks to (4.7), (4.11) and Lemma 2.1, we deduce that

$$
\begin{aligned}
& \sigma\left(t_{m}\right)\left(\left\|e_{\mathbf{u}}^{m}\right\|_{1}^{2}+\frac{v}{2}\left\|A_{h} e_{\mathbf{u}}^{m}\right\|_{0}^{2} \Delta t\right)+\Delta t \sum_{n=3}^{m} \sigma\left(t_{n}\right)\left(\frac{1}{2}\left\|d_{t} e_{\mathbf{u}}^{n}\right\|_{1}^{2} \Delta t+v\left\|A_{h} \bar{e}_{\mathbf{u}}^{n}\right\|_{0}^{2}\right) \leq C \Delta t \\
& \sigma\left(t_{m}\right)\left(\left\|e_{T}^{m}\right\|_{1}^{2}+\frac{P r^{-1} v}{2}\left\|A_{h} e_{T}^{m}\right\|_{0}^{2} \Delta t\right)+\Delta t \sum_{n=3}^{m} \sigma\left(t_{n}\right)\left(\frac{1}{2}\left\|d_{t} e_{T}^{n}\right\|_{1}^{2} \Delta t\right. \\
& \left.\quad+\operatorname{Pr}^{-1} v\left\|A_{h} \bar{e}_{T}^{n}\right\|_{0}^{2}\right) \leq C \Delta t
\end{aligned}
$$


Choosing $\mathbf{v}_{h}=2 d_{t} e_{\mathbf{u}}^{n} \Delta t \in V_{h}, q_{h}=0, \psi_{h}=0$ with $\alpha=1$ in (4.2), for all $3 \leq n \leq N$, by the similar proof as Theorem 4.2, we have

$$
\begin{gathered}
v\left\|e_{\mathbf{u}}^{n}\right\|_{1}^{2}-v\left\|e_{\mathbf{u}}^{n-1}\right\|_{1}^{2}+\frac{5}{8}\left\|d_{t} e_{\mathbf{u}}^{n}\right\|_{0}^{2} \Delta t-\frac{1}{8}\left\|d_{t} e_{\mathbf{u}}^{n-1}\right\|_{0}^{2} \Delta t \\
\leq \frac{v}{2} b_{n-1}\left\|e_{\mathbf{u}}^{n-1}\right\|_{1}^{2} \Delta t+\frac{v}{2} c_{n-1}\left\|e_{\mathbf{u}}^{n-2}\right\|_{1}^{2} \Delta t+16\left\|P_{h} e_{n}\right\|_{0}^{2} \Delta t .
\end{gathered}
$$

In the same way, taking $\psi_{h}=2 d_{t} e_{T}^{n} \Delta t \in W_{h}, \mathbf{v}_{h}=0, q_{h}=0$ with $\alpha=1$ in (4.2), for all $3 \leq n \leq N$, it holds

$$
\begin{aligned}
& \operatorname{Pr}^{-1} v\left\|e_{T}^{n}\right\|_{1}^{2}-\operatorname{Pr}^{-1} v\left\|e_{T}^{n-1}\right\|_{1}^{2}+\frac{5}{8}\left\|d_{t} e_{T}^{n}\right\|_{0}^{2} \Delta t-\frac{1}{8}\left\|d_{t} e_{T}^{n-1}\right\|_{0}^{2} \Delta t \\
\leq & \frac{\operatorname{Pr}^{-1} v}{2} b_{n-1}\left\|e_{T}^{n-1}\right\|_{1}^{2} \Delta t+\frac{\operatorname{Pr}^{-1} v}{2} c_{n-1}\left\|e_{T}^{n-2}\right\|_{1}^{2} \Delta t+16\left\|P_{h} e_{n}^{\prime}\right\|_{0}^{2} \Delta t .
\end{aligned}
$$

Multiplying by $\sigma^{2}\left(t_{n}\right)$ in (4.33), using (4.26), (4.32a), Theorems 2.2, 4.1, 4.2, 4.3 and summing from $n=3$ to $m$, we have

$$
\begin{aligned}
& \quad v \sigma^{2}\left(t_{m}\right)\left\|e_{\mathbf{u}}^{m}\right\|_{1}^{2}+\Delta t \sum_{n=3}^{m} \sigma^{2}\left(t_{n}\right)\left\|d_{t} e_{\mathbf{u}}^{n}\right\|_{0}^{2} \\
& \leq 4 \Delta t \sum_{n=2}^{m-1} \widehat{d}_{n} v \sigma^{2}\left(t_{n}\right)\left\|e_{\mathbf{u}}^{n}\right\|_{1}^{2}+c \Delta t \sum_{n=2}^{m-1} v \sigma\left(t_{n}\right)\left\|\bar{e}_{\mathbf{u}}^{n}+\frac{1}{2} d_{t} e_{\mathbf{u}}^{n} \Delta t\right\|_{1}^{2} \\
& \quad+v \sigma^{2}\left(t_{2}\right)\left\|e_{\mathbf{u}}^{2}\right\|_{1}^{2}+\frac{1}{8} \sigma^{2}\left(t_{2}\right)\left\|d_{t} e_{\mathbf{u}}^{2}\right\|_{0}^{2} \Delta t+c \Delta t \sum_{n=3}^{m} \sigma^{2}\left(t_{n}\right)\left\|P_{h} e_{n}\right\|_{0}^{2} \\
& \leq C \Delta t^{2}+4 \Delta t \sum_{n=2}^{m-1} \widehat{d}_{n} v \sigma^{2}\left(t_{n}\right)\left\|e_{\mathbf{u}}^{n}\right\|_{1}^{2} .
\end{aligned}
$$

Similarly, multiplying by $\sigma^{2}\left(t_{n}\right)$ in (4.34) and summing from $n=3$ to $m$, by (4.28), (4.32b), Theorems 2.2, 4.1, 4.2, 4.3, we have

$$
\begin{aligned}
& \quad v \sigma^{2}\left(t_{m}\right)\left\|e_{T}^{m}\right\|_{1}^{2}+\Delta t \sum_{n=3}^{m} \sigma^{2}\left(t_{n}\right)\left\|d_{t} e_{T}^{n}\right\|_{0}^{2} \\
& \leq c \Delta t \sum_{n=2}^{m-1} v \sigma\left(t_{n}\right)\left\|\bar{e}_{T}^{n}+\frac{1}{2} d_{t} e_{T}^{n} \Delta t\right\|_{1}^{2}+P r^{-1} v \sigma^{2}\left(t_{2}\right)\left\|e_{T}^{2}\right\|_{1}^{2} \\
& \quad+\frac{1}{8} \sigma^{2}\left(t_{2}\right)\left\|d_{t} e_{T}^{2}\right\|_{0}^{2} \Delta t+4 \Delta t \sum_{n=2}^{m-1} \widetilde{d}_{n} \operatorname{Pr}^{-1} v \sigma^{2}\left(t_{n}\right)\left\|e_{T}^{n}\right\|_{1}^{2}+c \Delta t \sum_{n=3}^{m} \sigma^{2}\left(t_{n}\right)\left\|P_{h} e_{n}^{\prime}\right\|_{0}^{2} \\
& \leq C \Delta t^{2}+4 \Delta t \sum_{n=2}^{m-1} \widetilde{d}_{n} \operatorname{Pr}^{-1} v \sigma^{2}\left(t_{n}\right)\left\|e_{T}^{n}\right\|_{1}^{2} .
\end{aligned}
$$

Applying Lemma 2.1 to (4.35) and (4.36), we deduce (4.31). 
Now, we provide the error estimate of $p_{h}^{n}$ with nonsmooth initial data. By (A3), (2.4), (4.2) and Lemma 2.2, we have

$$
\left\|e_{p}^{n}\right\|_{0} \leq c\left\|d_{t} e_{\mathbf{u}}^{n}\right\|_{0}+v\left\|\bar{e}_{\mathbf{u}}^{n}\right\|_{1}+c\left\|e_{\mathbf{u}}^{n-1}\right\|_{1}\left(\left\|\mathbf{u}_{h}\left(t_{n-1}\right)\right\|_{1}+\left\|\mathbf{u}_{h}^{n-1}\right\|_{1}\right)+c\left\|e_{n}\right\|_{0} .
$$

Thanks to Theorems 2.2 and 3.2, one finds

$$
\Delta t \sigma^{2}\left(t_{n}\right)\left\|e_{p}^{n}\right\|_{0}^{2} \leq C \Delta t \sigma^{2}\left(t_{n}\right)\left(\left\|d_{t} e_{\mathbf{u}}^{n}\right\|_{0}^{2}+\left\|\bar{e}_{\mathbf{u}}^{n}\right\|_{1}^{2}+\left\|e_{\mathbf{u}}^{n-1}\right\|_{1}^{2}+\left\|e_{n}\right\|_{0}^{2}\right) .
$$

Summing above inequality from 3 to $m$, by Theorems 4.1,4.2, and 4.3, we get

$$
\Delta t \sum_{n=1}^{m} \sigma^{2}\left(t_{n}\right)\left\|e_{p}^{n}\right\|_{0}^{2} \leq C \Delta t^{2}
$$

Thanks to Theorem 2.2 and the integral by parts, for all $1 \leq n \leq N$, we have

$$
\begin{aligned}
& \sigma^{2}\left(t_{n}\right)\left\|p_{h}\left(t_{n}\right)-p_{h}^{n}\right\|_{0}^{2} \Delta t \\
\leq & 2 \sigma^{2}\left(t_{n}\right)\left\|e_{p}^{n}\right\|_{0}^{2} \Delta t+2 \sigma^{2}\left(t_{n}\right)\left\|p_{h}\left(t_{n}\right)-\frac{1}{\Delta t} \int_{t_{n-1}}^{t_{n}} p_{h}(s) d s\right\|_{0}^{2} \Delta t \\
\leq & 2 \sigma^{2}\left(t_{n}\right)\left\|e_{p}^{n}\right\|_{0}^{2} \Delta t+8 \Delta t^{2} \int_{t_{n-1}}^{t_{n}} \sigma^{2}(s)\left\|p_{h s}(s)\right\|_{0} d s .
\end{aligned}
$$

Finally, summing above inequality from 1 to $m$, for $1 \leq m \leq N$, we deduce that

$$
\Delta t \sum_{n=1}^{m} \sigma^{2}\left(t_{n}\right)\left\|p_{h}\left(t_{n}\right)-p_{h}^{n}\right\|_{0}^{2} \leq C \Delta t^{2} .
$$

Thus, we complete the proof.

Combining Theorems 2.3, 4.3 and 4.4, we obtain the following main theoretical results.

Theorem 4.5. Under the conditions of (A1)-(A3) and (3.9), for all $t_{m} \in\left(0, T_{\text {time }}^{\text {final }}\right]$ it holds

$$
\begin{array}{ll}
\left\|\boldsymbol{u}\left(t_{m}\right)-\boldsymbol{u}_{h}^{m}, T\left(t_{m}\right)-T_{h}^{m}\right\|_{0} \leq C\left(\sigma^{-1}\left(t_{m}\right) \Delta t^{\frac{3}{2}}+\sigma^{-\frac{1}{2}}\left(t_{m}\right) h^{2}\right), & 1 \leq m \leq N \\
\left\|\boldsymbol{u}\left(t_{m}\right)-\boldsymbol{u}_{h}^{m}, T\left(t_{m}\right)-T_{h}^{m}\right\|_{1} \leq C\left(\sigma^{-1}\left(t_{m}\right) \Delta t+\sigma^{-\frac{1}{2}}\left(t_{m}\right) h\right), & 1 \leq m \leq N \\
\left(\Delta t \sum_{n=1}^{m} \sigma^{2}\left(t_{n}\right)\left\|p\left(t_{m}\right)-p_{h}^{m}\right\|_{0}^{2}\right)^{1 / 2} \leq C(\Delta t+h), & 1 \leq m \leq N
\end{array}
$$

\section{Numerical experiments}

In this section, we present some numerical results to verify the performances of the developed numerical schemes. In all experiments, the time-dependent natural convection 
problem is defined on a convex domain $\Omega=[0,1]^{2}$. The mesh consists of triangular elements that obtained by dividing $\Omega$ into subsquares of equal size and drawing the diagonal in each sub-square. The finite element spaces are adopted the MINI element for velocity and pressure and linear polynomial for temperature. The UMFPACK routine is used to solve the linear systems arising from the discrete algebraic systems. We set the final time $T_{\text {time }}^{\text {final }}=1$, the parameters $v, k, \operatorname{Pr}$ are 1 and $h=\Delta t$. For comparison, the numerical results of the following Crank-Nicolson scheme are provided.

Example 5.1 (The Crank-Nicolson scheme for the natural convection problem). Set the initial solutions $\mathbf{u}_{h}^{0}=\mathbf{u}_{0}, T_{h}^{0}=T_{0}$, for all $\left(\mathbf{v}_{h}, q_{h}, \psi_{h}\right) \in X_{h} \times M_{h} \times W_{h}$. Define the numerical solution $\left(\mathbf{u}_{h}^{n}, p_{h}^{n}, T_{h}^{n}\right) \in X_{h} \times M_{h} \times W_{h}, n=1, \cdots, N$ by

$$
\left\{\begin{array}{l}
\left(d_{t} \mathbf{u}_{h}^{n}, \mathbf{v}_{h}\right)+a\left(\overline{\mathbf{u}}_{h}^{n}, \mathbf{v}_{h}\right)-d\left(\mathbf{v}_{h}, p_{h}^{n}\right)+d\left(\mathbf{u}_{h}^{n}, q_{h}\right)+b\left(\overline{\mathbf{u}}_{h}^{n}, \overline{\mathbf{u}}_{h}^{n}, \mathbf{v}_{h}\right) \\
=\left(\overline{\mathbf{f}}\left(t_{n}\right), \mathbf{v}_{h}\right)-\kappa v^{2}\left(j \bar{T}_{h}^{n}, \mathbf{v}_{h}\right), \\
\left(d_{t} T_{h}^{n}, \psi_{h}\right)+\tilde{a}\left(\bar{T}_{h}^{n}, \psi_{h}\right)+\tilde{b}\left(\overline{\mathbf{u}}_{h}^{n}, \bar{T}_{h}^{n}, \psi_{h}\right)=\left(\bar{g}\left(t_{h}\right), \psi_{h}\right) .
\end{array}\right.
$$

It is well known that (5.1) is a classical second order nonlinear numerical scheme, we use the Newton iteration to treat the nonlinear terms. The iteration stop condition

$$
\sqrt{\left\|\mathbf{u}_{h}^{n}-\widetilde{\mathbf{u}}_{h}^{n}\right\|_{0}+\left\|T_{h}^{n}-\widetilde{T}_{h}^{n}\right\|_{0}} \leq 10^{-3}
$$

is used, $\widetilde{\mathbf{u}}_{h}^{n}$ and $\widetilde{T}_{h}^{n}$ are the $n$ th-level iterative solutions.

Firstly, we verify the established theoretical results of Theorem 4.5 with $H^{1}$-initial data. The boundary and initial conditions and body forces $f, g$ are given by the following exact solutions

$$
\begin{aligned}
u_{1}(x, y, t)= & \frac{5}{2} \pi \sin ^{\frac{5}{2}}(\pi x) \sin ^{\frac{3}{2}}(\pi y) \cos (\pi y) \cos (t), \\
u_{2}(x, y, t)= & -\frac{5}{2} \pi \sin ^{\frac{3}{2}}(\pi x) \cos (\pi x) \sin ^{\frac{5}{2}}(\pi y) \cos (t), \\
p(x, y, t)= & 10 \cos (\pi x) \cos (\pi y) \cos (t), \\
T(x, y, t)= & \frac{5}{2} \pi \sin ^{\frac{5}{2}}(\pi x) \sin ^{\frac{3}{2}}(\pi y) \cos (\pi y) \cos (t) \\
& -\frac{5}{2} \pi \sin ^{\frac{3}{2}}(\pi x) \cos (\pi x) \sin ^{\frac{5}{2}}(\pi y) \cos (t),
\end{aligned}
$$

where the velocity $\mathbf{u}=\left(u_{1}, u_{2}\right)$ and the initial data $\left(\mathbf{u}_{0}(x, y), T_{0}(x, y)\right) \in H^{1}$ (see [18]).

In Table 1, we present the relative errors between the exact solutions and numerical solutions of scheme (3.1)-(3.2). From these data, we can see that the relative errors of velocity and temperature in $H^{1}$-norm become smaller and smaller as the mesh refines, and the convergence orders are $\mathcal{O}(h)$. The error orders of pressure are nearly $\mathcal{O}\left(h^{1.6}\right)$, which show some superconvergences, the reason may lie in the smoothness of pressure. Moreover, the error order for $\mathbf{u}$ and $T$ in $L^{2}$-norm is of the order of $\mathcal{O}\left(h^{1.6}\right)$ as the mesh 
Table 1: The numerical results of the Crank-Nicolson/Explicit scheme with $H^{1}$-initial data.

\begin{tabular}{||ccccccc||}
\hline$\Delta t$ & $\frac{\left\|\mathbf{u}-\mathbf{u}_{h}^{n}\right\|_{0}}{\|\mathbf{u}\|_{0}}$ & Rate & $\frac{\left\|\nabla\left(\mathbf{u}-\mathbf{u}_{h}^{n}\right)\right\|_{0}}{\|\nabla \mathbf{u}\|_{0}}$ & Rate & $\frac{\left\|p-p_{h}^{n}\right\|_{0}}{\|p\|_{0}}$ & Rate \\
\hline 0.1 & 0.067019 & - & 0.362041 & - & 0.277928 & - \\
0.05 & 0.0184294 & 1.8626 & 0.187497 & 0.9493 & 0.0900259 & 1.6263 \\
0.025 & 0.00565494 & 1.7044 & 0.0939339 & 0.9971 & 0.0302961 & 1.5712 \\
0.0125 & 0.00186265 & 1.6022 & 0.0468949 & 1.0022 & 0.0104063 & 1.5417 \\
0.00625 & 0.000674092 & 1.4663 & 0.0234641 & 0.9990 & 0.00373707 & 1.4775 \\
$\Delta t$ & $\frac{\left\|\nabla\left(T-T_{h}^{n}\right)\right\|_{0}}{\|\nabla T\|_{0}}$ & Rate & $\frac{\left\|T-T_{h}^{n}\right\|_{0}}{\|T\|_{0}}$ & Rate & $\mathrm{CPU}(\mathrm{S})$ & \\
\hline 0.1 & 0.0326752 & - & 0.181239 & - & 1.45 & \\
0.05 & 0.00945539 & 1.7890 & 0.0906668 & 0.9992 & 7.03 & \\
0.025 & 0.00294663 & 1.6821 & 0.0453439 & 0.9997 & 58.87 & \\
0.0125 & 0.001018157 & 1.5331 & 0.0226746 & 0.9998 & 472.86 & \\
0.00625 & 0.000371619 & 1.4541 & 0.0113403 & 0.9996 & 4584.74 & \\
\hline
\end{tabular}

Table 2: The numerical results of the Crank-Nicolson scheme with $H^{1}$-initial data.

\begin{tabular}{||ccccccc||}
\hline$\Delta t$ & $\frac{\left\|\mathbf{u}-\mathbf{u}_{h}^{n}\right\|_{0}}{\|\mathbf{u}\|_{0}}$ & Rate & $\frac{\left\|\nabla\left(\mathbf{u}-\mathbf{u}_{h}^{n}\right)\right\|_{0}}{\|\nabla \mathbf{u}\|_{0}}$ & Rate & $\frac{\left\|p-p_{h}^{n}\right\|_{0}}{\|p\|_{0}}$ & Rate \\
\hline 0.1 & 0.0675168 & - & 0.323773 & - & 0.280633 & - \\
0.05 & 0.0187475 & 1.8485 & 0.161803 & 1.0007 & 0.0891139 & 1.6550 \\
0.025 & 0.00543686 & 1.7859 & 0.0837612 & 0.9449 & 0.0300184 & 1.5698 \\
0.0125 & 0.00176032 & 1.6269 & 0.0429238 & 0.9645 & 0.0104925 & 1.5165 \\
0.00625 & 0.000633639 & 1.4741 & 0.0228389 & 0.9103 & 0.00377609 & 1.4744 \\
\hline$\Delta t$ & $\frac{\| \nabla\left(T-T_{h}^{n} \|_{0}\right.}{\|\nabla T\|_{0}}$ & Rate & $\frac{\left\|T-T_{h}^{n}\right\|_{0}}{\|T\|_{0}}$ & Rate & $\mathrm{CPU}(\mathrm{S})$ & \\
\hline 0.1 & 0.0321728 & - & 0.181049 & - & 1.83 & \\
0.05 & 0.00936417 & 1.7806 & 0.0906609 & 0.9978 & 9.89 & \\
0.025 & 0.00284727 & 1.7176 & 0.0453435 & 0.9996 & 169.71 & \\
0.0125 & 0.000981062 & 1.5372 & 0.0226746 & 0.9998 & 1191.89 & \\
0.00625 & 0.000341612 & 1.5220 & 0.0113402 & 0.9996 & 16858.5 & \\
\hline
\end{tabular}

sizes reduce with $H^{1}$-initial data, which confirms the Theorem 4.5 well. In contrast, the relative errors obtained from the Crank-Nicolson $(\mathrm{CN})$ scheme (5.1) are presented in Table 2. Compared with Table 1, we can see that the accuracy of Crank-Nicolson/Explicit scheme is comparable with that of the CN scheme (5.1) with the same time steps, but the Crank-Nicolson/Explicit scheme can save 60\% CPU time than the Crank-Nicolson scheme.

Next, we consider the influences of the initial data for the numerical results, and choose the following $\mathrm{H}^{2}$-smooth initial data.

$$
\begin{aligned}
& u_{1}(x, y, t)=2 \pi \sin ^{2}(\pi x) \sin (\pi y) \cos (\pi y) \cos (t), \\
& u_{2}(x, y, t)=-2 \pi \sin (\pi x) \cos (\pi x) \sin ^{2}(\pi y) \cos (t), \\
& p(x, y, t)=10 \cos (\pi x) \cos (\pi y) \cos (t),
\end{aligned}
$$




$$
\begin{aligned}
T(x, y, t)= & 2 \pi \sin ^{2}(\pi x) \sin (\pi y) \cos (\pi y) \cos (t) \\
& -2 \pi \sin (\pi x) \cos (\pi x) \sin ^{2}(\pi y) \cos (t) .
\end{aligned}
$$

We present the relative errors of the Crank-Nicolson/Explicit scheme and the CrankNicolson scheme for the natural convection problem with $H^{2}$-initial data in Tables 3-4. From these data, we know that the time convergence orders of velocity and temperature in $L^{2}$-norm of both numerical schemes are 2 , which confirm the well-known theoretical analysis [19] well.

Finally, combining the data provided in Tables 1-2, we can see that the relative errors become smaller and smaller as the time step decreases. The higher smoothness of the initial data, the more accurate of numerical solution is obtained. The error decreases more slowly as the time steps less than 0.025 with nonsmooth initial data, while there are no influences for the accuracy of numerical solutions with $\mathrm{H}^{2}$-initial data. Another interesting

Table 3: The numerical results of the Crank-Nicolson/Explicit scheme with $H^{2}$-initial data.

\begin{tabular}{||ccccccc||}
\hline$\Delta t$ & $\frac{\left\|\mathbf{u}-\mathbf{u}_{h}^{n}\right\|_{0}}{\|\mathbf{u}\|_{0}}$ & Rate & $\frac{\left\|\nabla\left(\mathbf{u}-\mathbf{u}_{h}^{n}\right)\right\|_{0}}{\|\nabla \mathbf{u}\|_{0}}$ & Rate & $\frac{\left\|p-p_{h}^{n}\right\|_{0}}{\|p\|_{0}}$ & Rate \\
\hline 0.1 & 0.0690326 & - & 0.361623 & - & 0.280436 & - \\
0.05 & 0.0172267 & 2.0026 & 0.181268 & 0.9964 & 0.0890037 & 1.6557 \\
0.025 & 0.00420379 & 2.0349 & 0.0915812 & 0.9850 & 0.0299049 & 1.5735 \\
0.0125 & 0.00107255 & 1.9706 & 0.0468918 & 0.9657 & 0.0102821 & 1.5402 \\
0.00625 & 0.000267906 & 2.0012 & 0.0234581 & 0.9992 & 0.00360688 & 1.5113 \\
\hline$\Delta t$ & $\frac{\left\|\nabla\left(T-T_{h}^{n}\right)\right\|_{0}}{\|\nabla T\|_{0}}$ & Rate & $\frac{\left\|T-T_{h}^{n}\right\|_{0}}{\|T\|_{0}}$ & Rate & CPU(S) & \\
\hline 0.1 & 0.0374768 & - & 0.181012 & - & 1.78 & \\
0.05 & 0.00938669 & 1.9973 & 0.0906547 & 0.9976 & 8.72 & \\
0.025 & 0.00224005 & 2.0671 & 0.0453404 & 0.9996 & 79.11 & \\
0.0125 & 0.000582718 & 1.9437 & 0.0226721 & 0.9999 & 624.07 & \\
0.00625 & 0.000145659 & 2.0002 & 0.0113362 & 1.0001 & 5226.82 & \\
\hline
\end{tabular}

Table 4: The numerical results of the Crank-Nicolson scheme with $H^{2}$-initial data.

\begin{tabular}{||ccccccc||}
\hline$\Delta t$ & $\frac{\left\|\mathbf{u}-\mathbf{u}_{h}^{n}\right\|_{0}}{\|\mathbf{u}\|_{0}}$ & Rate & $\frac{\left\|\nabla\left(\mathbf{u}-\mathbf{u}_{h}^{n}\right)\right\|_{0}}{\|\nabla \mathbf{u}\|_{0}}$ & Rate & $\frac{\left\|p-p_{h}^{n}\right\|_{0}}{\|p\|_{0}}$ & Rate \\
\hline 0.1 & 0.0706045 & - & 0.323331 & - & 0.280751 & - \\
0.05 & 0.0175661 & 2.0070 & 0.161824 & 0.9986 & 0.0889595 & 1.6581 \\
0.025 & 0.00441545 & 1.9922 & 0.0821415 & 0.9782 & 0.0298910 & 1.5734 \\
0.0125 & 0.00110449 & 1.9992 & 0.0424163 & 0.9535 & 0.0103573 & 1.5291 \\
0.00625 & 0.000266768 & 2.0497 & 0.0218331 & 0.9581 & 0.00362797 & 1.5134 \\
\hline$\Delta t$ & $\frac{\left\|\nabla\left(T-T_{h}^{n}\right)\right\|_{0}}{\|\nabla T\|_{0}}$ & Rate & $\frac{\left\|T-T_{h}^{n}\right\|_{0}}{\|T\|_{0}}$ & Rate & $\mathrm{CPU}(\mathrm{S})$ & \\
\hline 0.1 & 0.0368658 & - & 0.181034 & - & 13.627 & \\
0.05 & 0.00930105 & 1.9868 & 0.0906586 & 0.9977 & 222.52 & \\
0.025 & 0.00232981 & 1.9972 & 0.0453414 & 0.9996 & 1101.86 & \\
0.0125 & 0.000582625 & 1.9996 & 0.0226721 & 0.9999 & 6460.91 & \\
0.00625 & 0.000145651 & 2.0001 & 0.0113362 & 1.0001 & 21095.01 & \\
\hline
\end{tabular}


phenomenon is the computational cost of solving the discrete algebraic equations. The used CPU time with $H^{1}$-initial data less than that with $H^{2}$-initial data in both the CrankNicolson/Explicit scheme and the Crank-Nicolson scheme. Therefore, we conclude that the smoothness of the initial data has important influences on both the theoretical results and the computational cost.

\section{Acknowledgements}

The authors would like to thank the referees for the helpful suggestions. This work was supported by NSF of China (No. 11971152).

\section{References}

[1] R. A. Adams, Sobolev Spaces, Academic Press, New York, 1975.

[2] M. BERCOVIER, AND O. PIRONNEAU, Error estimates for finite element solution of the Stokes problem in the primitive variables, Numer. Math., 33 (1979), pp. 211-224.

[3] B. Bialecki, M. Ganesh, AND K. MustaPha, Crank-Nicolson Petrov-Galerkin method with quadrature for semi-linear parabolic problems, Numer. Meth. Partial Differential Equations, 21 (2005), pp. 918-937.

[4] F. BREZZI, AND M. Fortin, Mixed and Hybrid Finite Element Methods, Springer-Verlag, New York, 1991.

[5] J. BULI, AND Y. L. XING, Local discontinuous Galerkin methods for the Boussinesq coupled BBM system, J. Sci. Comput., 75 (2017), pp. 1-24.

[6] P. G. CiARLeT, The Finite Element Method for Elliptic Problems, North-Holland, Amsterdam, 1978.

[7] H. Dallmann, AND D. ARndT, Stabilized finite element methods for the Oberbeck-Boussinesq model, J. Sci. Comput., 69 (2016), pp. 244-273.

[8] M. DUMBSER, AND M. FACCHINI, A space-time discontinuous Galerkin method for Boussinesqtype equations, Appl. Math. Comput., 272 (2016), pp. 336-346.

[9] X. L. FenG, AND Y. N. HE, Convergence of the Crank-Nicolson/Newton scheme for nonlinear parabolic problems, Acta Math. Sci., 1 (2016), pp. 124-138.

[10] X. L.FenG, T. TANG, AND J. YANG, Stabilized Crank-Nicolson/Adams-Bashforth schemes for phase field models, E. Asian J. Appl. Math., 3 (2013), pp. 59-80.

[11] V. GiRAUlt, AND P. A. RAVIART, Finite Element Method for Navier-Stokes Equations: Theory and Algorithms, Springer-Verlag, Berlin, Heidelberg, 1987.

[12] Y. N. HE, Two level method based on finite element and Crank-Nicolson extrapolation for the timedependent Navier-Stokes equations, SIAM J. Numer. Anal., 41 (2003), pp. 1263-1285.

[13] Y. N. HE, Stability and error analysis for a spectral Galerkin method for the Navier-Stokes equations with $H^{2}$ or $H^{1}$ initial data, Numer. Meth. Partial Differential Equations, 21 (2005), pp. 875-904.

[14] Y. N. HE, AND W. W. Sun, Stability and convegence of the Crank-Nicolson/Adams-Bashforth scheme for the time-dependent Navier-Stokes equations, SIAM J. Numer. Anal., 45 (2007), pp. 837-869.

[15] Y. N. HE, Euler implicit/explicit scheme for the 2D time-dependent Navier-Stokes equations with smooth or non-smooth initial data, Math. Comput., 77 (2008), pp. 2097-2124. 
[16] Y. N. HE, The Crank-Nicolson/Adams-Bashforth scheme for the time-dependent Navier-Stokes equations with nonsmooth initial data, Numer. Meth. Partial Differential Equations, 28 (2012), pp. 155-187.

[17] Y. N. HE, Euler implicit/explicit iterative scheme for the stationary Navier-Stokes equations, Numer. Math., 123 (2013), pp. 67-96.

[18] Y. N. HE, P. Z. HUANG, AND X. L. FENG, $H^{2}$-Stability of the first order fully discrete schemes for the time-dependent Navier-Stokes equations, J. Sci. Comput., 62 (2105), pp. 230-264.

[19] J. G. HEYWOOD, AND R. RANNACHER, Finite element approximations of the nonstationary Navier-Stokes problem, Part IV: error estimates for second-order time discretization, SIAM J. Numer. Anal., 27 (1990), pp. 353-384.

[20] X. H. HU, P. Z. HUANG, AND X. L. FENG, A new mixed finite element method based on the Crank-Nicolson scheme for Burgers' equation, Appl. Math. CZECH, 61 (2016), pp. 27-45.

[21] P. Z. HUANG, An efficient two-level finite element algorithm for the natural convection equations, Appl. Numer. Math., (118) (2017), pp. 75-86.

[22] P. Z. HuAng, X. L. FENG, AND D. M. LiU, A stabilized finite element method for the timedependent Stokes equations based on Crank-Nicolson scheme, Appl. Math. Model., 37 (2013), pp. 1910-1919.

[23] P. Z. HUANG, W. Q. LI, AND Z. Y. SI, Several iterative schemes for the stationary natural convection equations at different Rayleigh numbers, Numer. Meth. Partial Differential Equations, 31 (2015), pp. 761-776.

[24] F. LiU, W. P. SHI, AND F. F. WU, A lattice Boltzmann model for the generalized Boussinesq equation, Appl. Math. Comput., 274 (2016), pp. 331-342.

[25] Z. D. LUO, The Bases and Applications of Mixed Finite Element Methods, Chinese Science Press, Beijing, 2006, in Chinese.

[26] A. A. MOHAmad, AND A. KuZmin, A critical evaluation of force term in lattice Boltzmann method, natural convection problem, Int. J. Heat. Mass. Trans., 53 (2010), pp. 990-996.

[27] N. PANDA, C. DAWSON, AND Y. ZHANG, Discontinuous Galerkin methods for solving Boussinesq-Green-Naghdi equations in resolving non-linear and dispersive surface water waves, J. Comput. Phys., 273 (2014), pp. 572-588.

[28] H. Y. SU, X. L. FENG, AND Y. N. HE, Defect-correction finite element method based on CrankNicolson extrapolation scheme for the transient conduction-convection problem with high Reynolds number, Int. Commun. Heat Mass, 81 (2017), pp. 229-249.

[29] C. TAYLOR, AND P. HOOD, A numerical solution of the Navie-Stokes equations using the finite element technique, Comput. Fluids, 1 (1973), pp. 73-100.

[30] R. TEmAM, Navier-Stokes Equations: Theory and Numerical Analysis (Third edition), North-Holland, Amsterdam, New York, Oxford, 1984.

[31] T. ZHANG, J. J. Jin, AND T. JiAnG, The decoupled Crank-Nicolson/Adams-Bashforth scheme for the Boussinesq equations with nonsmooth initial data, Appl. Math. Comput., 337 (2018), pp. 234-266.

[32] T. ZHANG, J. Y. YUAN, AND Z. Y. SI, Decoupled two grid finite element method for the timedependent natural convection problem I: spatial discretization, Numer. Meth. Partial Differential Equations, 31 (2015), pp. 2135-2168.

[33] T. ZHANG, X. ZHAO AND P. Z. HuANG, Decoupled two level finite element methods for the steady natural convection problem, Numer. Algorithms, 68 (2015), pp. 837-866. 